\title{
Neutrino Mass Hierarchy
}

\author{
X. Qian ${ }^{1 *}$ and P. Vogel $^{2 \dagger}$ \\ ${ }^{1}$ Physics Department, Brookhaven National Laboratory, Upton, NY, USA \\ ${ }^{2}$ Kellogg Radiation Laboratory, California Institute of Technology, Pasadena, CA, USA
}

May 11, 2015

\begin{abstract}
The neutrino mass hierarchy, i.e., whether the $\nu_{3}$ neutrino mass eigenstate is heavier or lighter than the $\nu_{1}$ and $\nu_{2}$ mass eigenstates, is one of the remaining undetermined fundamental features of the neutrino Standard Model. Its determination would represent an important step in the formulation of the generalized model, and would have a profound impact on the quest of the nature of neutrinos (Dirac or Majorana) and the search for a theory of flavor. In this review, we summarize the status of experimental and theoretical work in this field and explore the future opportunities that emerge in light of the recently discovered non-zero and relatively large third neutrino mixing angle $\theta_{13}$.
\end{abstract}

\section{Contents}

1 Introduction 2

2 Various Methods to Determine the Mass Hierarchy 9

2.1 The "Large" Third Neutrino Mixing Angle $\theta_{13} \ldots \ldots$. . . . . . . . . . . . . . . . . . . . . 9

2.2 Mass Hierarchy from Accelerator Neutrinos Appearance . . . . . . . . . . . . . . . . . . 10

2.3 Mass Hierarchy trom Atmospheric neutrinos . . . . . . . . . . . . . . . . . . . . . . . . 15

2.4 Mass Hierarchy from Reactor Antineutrinos . . . . . . . . . . . . . . . . . . . . . . . . 19

2.5 Interpretation of Mass Hierarchy Sensitivity . . . . . . . . . . . . . . . . . . . . . . . 22

2.5 .1 Bayesian Approach to the Mass Hierarchy Determination . . . . . . . . . . . . . 24

2.5 .2 Frequentist Approach to the Mass Hierarchy Determination . . . . . . . . . . . 24

$\begin{array}{llr}3 & \text { Experiments } & 26\end{array}$

3.1 Current Status . . . . . . . . . . . . . . . . . . . . . . . . . . . . 27

3.1.1 Atmospheric neutrino results from Super-Kamiokande . . . . . . . . . . . . . . . 27

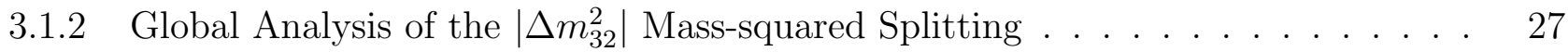

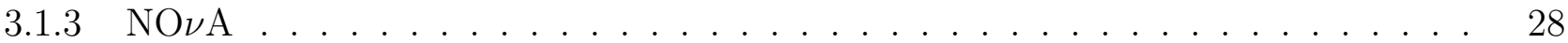

3.2 Proposed and Planned Experiments . . . . . . . . . . . . . . . . . . . . . . . . . . . . . . . . 29

*Email: xqian@bnl.gov

†Email:pvogel@caltech.edu 
3.2 .1 DUNE/LBNF . . . . . . . . . . . . . . . . . . . . . . . . . 29

3.2 .2 PINGU, ORCA, INO and Hyper Kamiokande . . . . . . . . . . . . . . . . 33

3.2 .3 Medium baseline reactor experiments: JUNO and RENO-50 . . . . . . . . . 37

4 Summary and Outlook

5 Acknowledgments

\section{Introduction}

Intense experimental and global analysis effort over the last several decades led to only a very small number of observations signifying that the standard model of elementary particles is incomplete and that ultimately it requires a generalization. Among them the existence of non-vanishing neutrino masses has a prominent position. Despite the obvious difficulty of experiments involving neutrinos, it is now clear beyond any doubt that neutrinos have masses, albeit tiny, and that they mix, unlike the assumptions of the standard model. Thanks to the discoveries of the last twenty years, we now have a simple three-flavor description of these new phenomena that explains most of the data. Despite the success, this is not the end of the story. Further experimental and theoretical effort is needed so that the next step, formulation of a new theory that encompasses the new insight into the neutrino physics, can be accomplished.

Experiments with solar, atmospheric, reactor, and accelerator neutrinos have determined, with remarkable accuracy, most of the necessary parameters that describe the three-generation oscillations. However, several crucial pieces are still missing: the neutrino mass hierarchy (or the ordering of the neutrino masses), the magnitude of the $\mathrm{CP}$ (charge and parity) phase $\delta_{C P}$, the absolute scale of the neutrino mass, and whether neutrinos are Dirac or Majorana fermions. This review is devoted to the problem of the neutrino mass hierarchy.

In the following, we assume that the neutrinos $\nu_{e}, \nu_{\mu}$ and $\nu_{\tau}$ (so-called flavor eigenstates) that couple with the gauge bosons $W^{ \pm}$through weak interactions are coherent superpositions of three mass eigenstates $\nu_{i}, i=1,2,3$, i.e.

$$
\left(\begin{array}{c}
\nu_{e} \\
\nu_{\mu} \\
\nu_{\tau}
\end{array}\right)=\left(\begin{array}{ccc}
U_{e 1} & U_{e 2} & U_{e 3} \\
U_{\mu 1} & U_{\mu 2} & U_{\mu 3} \\
U_{\tau 1} & U_{\tau 2} & U_{\tau 3}
\end{array}\right) \cdot\left(\begin{array}{c}
\nu_{1} \\
\nu_{2} \\
\nu_{3}
\end{array}\right),
$$

where $U$ is a unitary $3 \times 3$ matrix. In other words, we assume that there are three flavor and mass neutrino states and dismiss, for the purpose of this work, the possible existence of additional sterile neutrinos. This matrix implicitly contains possible charged lepton mixing; it is assumed that the corresponding charged lepton matrix matrix is diagonal in the flavor basis.

The lepton mixing matrix $U$ [1, [2, B] (usually denoted as the PMNS matrix for Pontecorvo, Maki, Nakagawa and Sakata who were the pioneers of the field) can be parametrized in terms of the three angles $\theta_{13}, \theta_{23}$, and $\theta_{12}$ and the $C P$ phase $\delta_{C P}$.

$$
\begin{aligned}
U & =\left(\begin{array}{ccc}
1 & 0 & 0 \\
0 & c_{23} & s_{23} \\
0 & -s_{23} & c_{23}
\end{array}\right) \cdot\left(\begin{array}{ccc}
c_{13} & 0 & s_{13} e^{-i \delta_{C P}} \\
0 & 1 & 0 \\
-s_{13} e^{i \delta_{C P}} & 0 & c_{13}
\end{array}\right) \cdot\left(\begin{array}{ccc}
c_{12} & s_{12} & 0 \\
-s_{12} & c_{12} & 0 \\
0 & 0 & 1
\end{array}\right) \cdot\left(\begin{array}{ccc}
e^{i \alpha} & 0 & 0 \\
0 & e^{i \beta} & 0 \\
0 & 0 & 1
\end{array}\right) \\
& =\left(\begin{array}{ccc}
-c_{13} c_{12} & c_{13} e^{-i \delta_{C P}} \\
-c_{23}-s_{13} s_{23} c_{12} e^{i \delta_{C P}} & c_{23} c_{12}-s_{13} s_{23} s_{12} e^{i \delta_{C P}} & c_{13} s_{23} \\
s_{23} s_{12}-s_{13} c_{23} c_{12} e^{i \delta_{C P}} & -s_{23} c_{12}-s_{13} c_{23} s_{12} e^{i \delta_{C P}} & c_{13} c_{23}
\end{array}\right) \cdot\left(\begin{array}{ccc}
e^{i \alpha} & 0 & 0 \\
0 & e^{i \beta} & 0 \\
0 & 0 & 1
\end{array}\right)
\end{aligned}
$$

where e.g. $c_{i j}=\cos \theta_{i j}$ and $s_{i j}=\sin \theta_{i j}$, etc. Here, $\alpha$ and $\beta$ (both unknown at the present) are so-called Majorana phases that are decoupled from the phenomenon of neutrino oscillation. 
Table 1: Neutrino oscillation parameters taken from Ref. [4]. For the atmospheric mass-squared splitting $\left(\Delta m_{3 x}^{2}\right)$, the best fit results for both the normal and the inverted mass hierarchy are shown. These values are used in all the following plots except where noted.

\begin{tabular}{|c|cc|}
\hline parameter & best fit value $\pm 1 \sigma$ & $3 \sigma$ range \\
\hline \hline $\sin ^{2} \theta_{12}$ & $0.304_{-0.012}^{+0.012}$ & $(0.270,0.344)$ \\
$\theta_{12}$ (degrees) & $33.48_{-0.74}^{+0.77}$ & $(31.30,35.90)$ \\
\hline $\sin ^{2} \theta_{23}$ & {$\left[0.451_{-0.001}^{+0.001}\right]$ or $0.577_{-0.035}^{+0.027}$} & $(0.385,0.644)$ \\
$\theta_{23}$ (degrees) & {$\left[42.2_{-0.1}^{+0.1}\right]$ or $49.4_{-2.0}^{+1.6}$} & $(38.4,53.3)$ \\
\hline $\sin ^{2} \theta_{13}$ & $0.0219_{-0.0011}^{+0.0010}$ & $(0.0188,0.0251)$ \\
$\theta_{13}$ (degrees) & $8.52_{-0.21}^{+0.20}$ & $(7.87,9.11)$ \\
\hline$\delta_{C P}($ degrees $)$ & $251_{-59}^{+67}$ & $(0,360)$ \\
\hline \hline$m_{21}^{2} \times 10^{-5} \mathrm{eV}^{2}$ & $7.50_{-0.17}^{+0.19}$ & $(7.03,8.09)$ \\
\hline$\left(\right.$ normal) $\Delta m_{31}^{2} \times 10^{-3} \mathrm{eV}^{2}$ & $+2.458_{-0.047}^{+0.046}$ & $(+2.325,+2.599)$ \\
$($ inverted $) \Delta m_{32}^{2} \times 10^{-3} \mathrm{eV}^{2}$ & $-2.448_{-0.047}^{+0.047}$ & $(-2.590,-2.307)$ \\
\hline
\end{tabular}

Table $\square$ summarizes the present knowledge of neutrino masses and mixings including neutrino mixing angles, the CP phase $\delta_{C P}$, and neutrino mass-squared differences $\Delta m_{i j}^{2}=m_{i}^{2}-m_{j}^{2}$, based on the recent fit [4] after the Neutrino 2014 conference [5]. A comparable result has also been obtained in Ref. [6]]. The pattern of neutrino masses and mixings is schematically shown in Fig. 1 . At the present time, we cannot decide whether the $\nu_{3}$ neutrino mass eigenstate is heavier or lighter than the $\nu_{1}$ and $\nu_{2}$ neutrino mass eigenstates in nature. The scenario, in which the $\nu_{3}$ is heavier, is referred to as the normal mass hierarchy $(\mathrm{NH})$. The other scenario, in which the $\nu_{3}$ is lighter, is referred to as the inverted mass hierarchy (IH).

Superficially, it appears that the difference between the two mass orderings depicted in Fig. $\square$ is striking, and hence it should be relatively easy to decide which one of them is realized in nature. As we show in detail further this is not the case and, in fact, the determination of the true mass hierarchy $(\mathrm{MH})$ is very challenging. However, despite the difficulties, it is vitally important to solve this problem, and correspondingly a great effort is devoted to its solution.

Lets first explain the nomenclature. Since the neutrinos $\nu_{1}$ has the largest component of the electron neutrino $\nu_{e}$ while $\nu_{3}$ has the smallest component of $\nu_{e}$, the normal hierarchy in some crude sense resembles the mass ordering of the charged leptons, hence it is denoted as 'normal' . Obviously, the inverted hierarchy represents the opposite situation. Note that the word hierarchy is used throughout this work even if the lightest neutrino mass $m_{L}$ ( $\nu_{1}$ for the $\mathrm{NH}$ and $\nu_{3}$ in the $\left.\mathrm{IH}\right)$ could be comparable to the other two masses, i.e. when all three masses are much larger than the $\sqrt{\Delta m_{i j}^{2}}$ (so called degenerate scenario).

Why is it important to determine the MH? The ultimate goal of all experiments and theories centered on neutrinos is to formulate the particle physics model that explains the observed neutrino masses and mixing patterns, and relates them to the well known charged lepton masses (and possibly to the quark masses and mixings). Clearly, explaining $\mathrm{MH}$ is a crucial part of that, and its determination would strengthen, or eliminate, roughly half of the proposed models.

Neutrino masses can be also constrained or ultimately determined by cosmology and astrophysics, where the sum of the masses, $m_{\text {cosmo }}=\Sigma m_{i}$ is the relevant quantity. So far upper limits on that sum, more and more restrictive, were reported [7]. Depending on the $\mathrm{MH}$, a lower limit of $m_{\text {cosmo }}$ is $\sim 0.05$ $\mathrm{eV}$ for $\mathrm{NH}$ and $\sim 0.1 \mathrm{eV}$ for IH. Thus, knowing the MH changes the lower limit by a factor of two, and correspondingly restrict the range of the sum. 
Most theoretical models of neutrino mass assume that neutrinos are massive Majorana fermions. The best way to test such a hypothesis is to search for the neutrinoless double beta decay $0 \nu \beta \beta$; its rate is proportional to the square of the effective neutrino mass $m_{e e}:=\left.|| U_{e 1}\right|^{2} m_{1}+\left|U_{e 2}\right|^{2} m_{2} e^{2 \alpha i}+\left|U_{e 3}\right|^{2} m_{3} e^{2 \beta i} \mid$. That quantity is restricted from below, $m_{e e} \geq 14 \mathrm{meV}$ (taking into account the $3 \sigma$ error bars of the oscillation parameters) for IH while $m_{e e}=0$ is possible for NH. Thus, if IH is realized in nature, the next generation of the $0 \nu \beta \beta$ experiments can decide whether neutrino are Majorana fermions or not.

\section{Neutrino Mass Hierarchy}

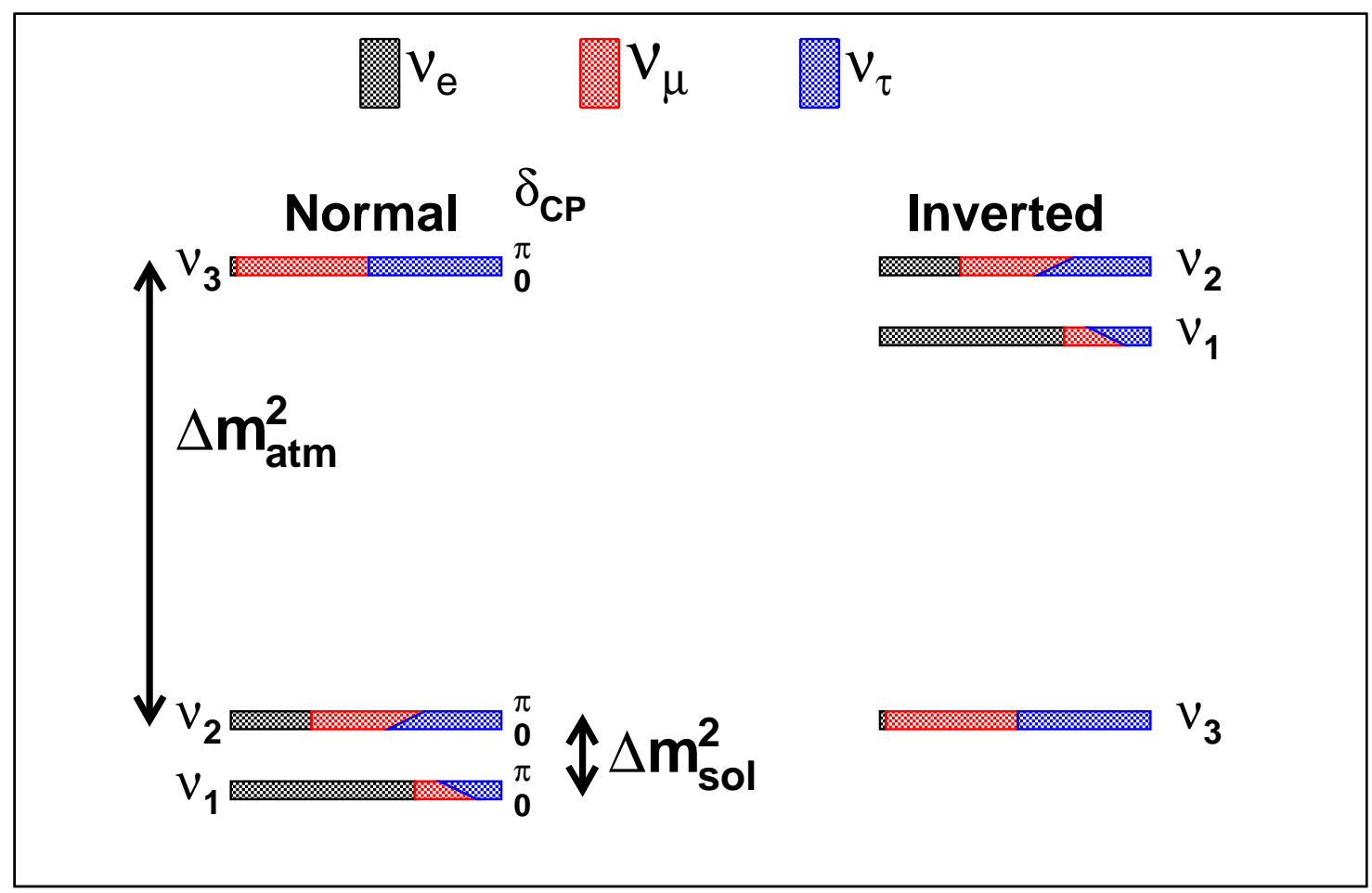

Figure 1: Pattern of neutrino masses for the normal and inverted hierarchies is shown as mass squared. Flavor composition of the mass eigenstates as the function of the unknown CP phase $\delta_{C P}$ is indicated. $\Delta m_{\text {atm }}^{2} \sim\left|\Delta m_{31}^{2}\right| \sim\left|\Delta m_{32}^{2}\right|$ and $\Delta m_{\text {sol }}^{2} \sim \Delta m_{21}^{2}$ stands for the atmospheric and the solar mass-squared splitting, respectively.

Similarly to most of the parameters describing neutrino mass and mixing, the neutrino $\mathrm{MH}$ can be accessed through the neutrino flavor oscillation. As shown in Table $\mathbb{D}$, there are two small parameters in the neutrino oscillation description; the mixing angle $\theta_{13}\left(\sin ^{2} \theta_{13} \sim 0.022\right)$ and the ratio $\Delta m_{21}^{2} / \Delta m_{31}^{2}$ $(\sim 3 \%)$. Due to this feature, most oscillation results are reasonably well described in the framework of mixing only two neutrinos, instead of three. In this case, the probability of flavor change in the vacuum and the oscillation length are given by

$$
P\left(\nu_{l} \rightarrow \nu_{l^{\prime}}\right)=\sin ^{2} 2 \theta \cdot \sin ^{2}\left(1.27 \cdot \frac{\Delta m^{2}\left(\mathrm{eV}^{2}\right) \cdot L(\mathrm{~m})}{E(\mathrm{MeV})}\right), L_{\text {vacuum }}(\mathrm{m})=\frac{2.48 \cdot E_{\nu}(\mathrm{MeV})}{\Delta m^{2}\left(\mathrm{eV}^{2}\right)}
$$

and, obviously, the sign of $\Delta m^{2}$ (the mass hierarchy) cannot be determined in such case.

Therefore, in order to determine $\mathrm{MH}$, i.e. to find effects that are sensitive to the sign of $\Delta m_{31}^{2}$ or $\Delta m_{32}^{2}$, one has to either go beyond the vacuum oscillation or go beyond the simple framework of two-neutrino mixing. Correspondingly, there are two direct ways to determine MH. In the first 
method, instead of the neutrino propagation in vacuum, one uses the propagation in matter where an additional phase from the interaction between neutrinos and the matter constituents makes the hierarchy determination possible. This effect is commonly referred to as the the Mikheyev-Smirnov-Wolfenstein (MSW) effect [8, 9, [10] or the matter effect. In the second method, one explores the aforementioned small difference $\left(\Delta m_{21}^{2}\right)$ between $\Delta m_{31}^{2}$ and $\Delta m_{32}^{2}$. In either case, carefully designed experiments are required. In the following, without going into details, we show the basic principles of both methods.

The oscillation phenomenon has its origin in the phase difference between the coherent components of the neutrino flavor eigenstates caused by the finite masses $m_{i}$ of the mass eigenstates $\nu_{i}(i=1,2,3)$. When neutrinos propagate in matter, an additional contribution to the phase appears. That phase affects the electron neutrinos and antineutrinos only, since only electron neutrinos/antineutrino interact with electrons in matter through the charged weak current $\left(W^{ \pm}\right.$-boson exchange). All three neutrino flavors interact with matter constituents through neutral current (Z-boson exchange) with same strength. Thus, these phases do not have effects on the neutrino oscillation. The corresponding effective potential and the matter oscillation length are

$$
V_{C}=\sqrt{2} G_{F} N_{e}, \quad L_{\text {matter }}=\frac{2 \pi}{\sqrt{2} G_{F} N_{e}}=\frac{1.7 \times 10^{7}(\mathrm{~m})}{\rho\left(\mathrm{g} / \mathrm{cm}^{3}\right) Y_{e}}
$$

where $G_{F}$ is the Fermi constant, $N_{e}$ is the electron density in matter, $\rho$ is the matter density, and $Y_{e} \sim 0.5$ is the electron fraction.

For the illustrative case of two flavors of neutrinos propagating in matter, the equation of motion for neutrinos in the flavor basis then has the form

$$
i \frac{d}{d x}\left(\begin{array}{c}
\nu_{e} \\
\nu_{\alpha}
\end{array}\right)=2 \pi\left(\begin{array}{cc}
-\kappa \frac{\cos 2 \theta}{L_{\text {vacuum }}+\frac{1}{L_{\text {matter }} \theta}} & \kappa \frac{\sin 2 \theta}{2 L_{\text {vacuum }}} \\
\kappa \frac{\sin }{2 L_{\text {vacuum }}} & 0
\end{array}\right)\left(\begin{array}{l}
\nu_{e} \\
\nu_{\alpha}
\end{array}\right)
$$

where $\kappa=\operatorname{sign}\left(m_{2}^{2}-m_{1}^{2}\right)$. For antineutrinos the sign in front of $1 / L_{\text {matter }}$ is reversed. Here one can see clearly that in matter, unlike in vacuum, the oscillation pattern depends on whether $m_{2}$ is larger or smaller than $m_{1}$. Note that the sign of $\Delta m_{21}^{2}$ can be compensated by the sign of the $\cos 2 \theta$, i.e. by the octant of the mixing angle $\theta$. The ordinary matter contains electrons, thus what really matters is whether $\nu_{e}$ is dominantly the lighter or the heavier one of the matter eigenstates $\left(\nu_{1}\right.$ or $\left.\nu_{2}\right)$.

Therefore, from the study of solar neutrinos, where the matter effects are crucial, we know that $\Delta m_{21}^{2}>0$, provided that we define, as it is customary, that $\nu_{1}$ is the mass eigenstate that is the dominant component of the electron neutrinos $\nu_{e}$. However, given the size of the matter oscillation length, it is clear that in order to use the matter oscillations in Earth to determine the sign of $\Delta m_{32}^{2}$ and $\Delta m_{31}^{2}$, very long baselines are needed.

Matter effect in earth may be used for the determination of $\mathrm{MH}$ in two ways. The first method utilizes the appearance of new neutrino flavors with accelerator neutrino sources, in practice the appearance of the electron neutrinos $\nu_{e}$ in a beam that begins as the muon neutrinos $\nu_{\mu}$. The probability that a beam of $\nu_{\mu}$ propagating in the matter of a constant electron density $N_{e}$ will be transformed into $\nu_{e}$ as a function of the neutrino energy $E_{\nu}$ and the neutrino travel distance $L$ is

$$
P\left(\nu_{\mu} \rightarrow \nu_{e}\right) \approx P_{0}+P_{\sin \delta}+P_{\cos \delta}+P_{3},
$$


where

$$
\begin{aligned}
P_{0} & =\sin ^{2} \theta_{23} \frac{\sin ^{2} 2 \theta_{13}}{(A-1)^{2}} \sin ^{2}[(A-1) \Delta], \\
P_{3} & =\alpha^{2} \cos ^{2} \theta_{23} \frac{\sin ^{2} 2 \theta_{12}}{A^{2}} \sin ^{2}(A \Delta), \\
P_{\sin \delta} & =-\alpha \frac{\sin 2 \theta_{12} \sin 2 \theta_{13} \sin 2 \theta_{23} \cos \theta_{13} \sin \delta_{C P}}{A(1-A)} \sin \Delta \sin (A \Delta) \sin [(1-A) \Delta], \\
P_{\cos \delta} & =\alpha \frac{\sin 2 \theta_{12} \sin 2 \theta_{13} \sin 2 \theta_{23} \cos \theta_{13} \cos \delta_{C P}}{A(1-A)} \cos \Delta \sin (A \Delta) \sin [(1-A) \Delta],
\end{aligned}
$$

and where

$$
\Delta=\frac{\Delta m_{31}^{2} L}{4 E_{\nu}}, \alpha=\frac{\Delta m_{21}^{2}}{\Delta m_{31}^{2}}, A=\sqrt{2} G_{F} N_{e} \frac{2 E_{\nu}}{\Delta m_{31}^{2}} .
$$

When the neutrinos are replaced by antineutrinos the signs of the CP phase $\delta_{C P}$ and of the matter effect signifying the parameter $A$ are reversed. Given known values of mixing angles, when measurements at optimally selected $L / E_{\nu}$ with both neutrinos and antineutrinos are performed, the matter effect and the $C P$ violation can be disentangled and the sign of $\mathrm{MH}$ (i.e. the sign of $\Delta$ ) can be determined. Fig. 2 shows the oscillation probabilities vs. $E_{\nu}$ at $1300 \mathrm{~km}$ baseline with distinguishing features of MH. A constant matter density is assumed. At $1300 \mathrm{~km}$, the difference in oscillation probabilities between a constant matter density and realistic earth density profile is negligible.

In addition, $\mathrm{MH}$ could be determined using the atmospheric neutrinos exposed to the matter effect by their propagation through Earth. Resonant oscillation [11, 12] occurs either for neutrinos in the case of $\mathrm{NH}$, or antineutrinos for IH. A detector that could resolve $\nu$ from $\bar{\nu}$ needs only to demonstrate for which state the resonance occurs. Detectors that can only determine neutrino flavor can take advantage of the difference in the atmospheric neutrino flux between $\nu$ and $\bar{\nu}$ and the differences in interaction cross sections at the detection to determine the hierarchy. Details of the needed strategy and the corresponding challenges with these two approaches will be described in the next section.

Beside the matter effect, $\mathrm{MH}$ can be determined by exploring the small difference $\left(\Delta m_{21}^{2}\right)$ between $\Delta m_{31}^{2}$ and $\Delta m_{32}^{2}$ in the three-flavor neutrino framework with neutrino and antineutrino disappearance. The survival probability for a neutrino of flavor $l$ in vacuum is given by

$$
P_{l l}=1-\left[A_{21}^{l} \sin ^{2} \Delta_{21}+A_{31}^{l} \sin ^{2} \Delta_{31}+A_{32}^{l} \sin ^{2} \Delta_{32}\right],
$$

where $A_{i j}^{l}=4\left|U^{l i}\right|^{2}\left|U^{l j}\right|^{2}$ and $\Delta_{i j}=\Delta m_{i j}^{2} L / 4 E_{\nu}$. This general formula, however, does not show explicitly the difference in the survival probability for the two hierarchies. In the case of practical importance, namely for the electron antineutrinos, the formula can be transformed into

$$
P_{e e}=1-2 s_{13}^{2} c_{13}^{2}-4 c^{4} 13 s_{12}^{2} c_{12}^{2} \sin ^{2} \Delta_{21}+2 s_{13}^{2} c_{13}^{2} \sqrt{1-4 s_{12}^{2} c_{12}^{2} \sin ^{2} \Delta_{21}} \cos \left(2 \Delta_{32} \pm \phi_{e e}\right),
$$

where the angle $\phi$ that characterizes the difference of the two hierarchies is

$$
\sin \phi_{e e}=\frac{c_{12}^{2} \sin 2 \Delta_{21}}{\sqrt{1-4 s_{12}^{2} c_{12}^{2} \sin ^{2} \Delta_{21}}}, \quad \cos \phi_{e e}=\frac{c_{12}^{2} \cos 2 \Delta_{21}+s_{12}^{2}}{\sqrt{1-4 s_{12}^{2} c_{12}^{2} \sin ^{2} \Delta_{21}}} .
$$

Here the \pm sign in the last term in Eq. (ए2) contains the information regarding the mass hierarchy. The plus (minus) sign corresponds to the normal (inverted) mass hierarchy. The analogous formula for the survival probability of the muon neutrinos is

$$
\begin{aligned}
P_{\mu \mu} & =1-2\left|U_{\mu 3}\right|^{2} \cdot\left(\left|U_{\mu 1}\right|^{2}+\left|U_{\mu 2}\right|^{2}\right)-4\left|U_{\mu 1}\right|^{2}\left|U_{\mu 2}\right|^{2} \sin ^{2} \Delta_{21} \\
& -4\left|U_{\mu 3}\right|^{2} \cdot\left(\sqrt{\left|U_{\mu 1}\right|^{4}+\left|U_{\mu 2}\right|^{4}+2\left|U_{\mu 1}\right|^{2}\left|U_{\mu 2}\right|^{2} \cos 2 \Delta_{21}}\right) \cdot \cos \left(2 \Delta_{32} \pm \phi_{\mu \mu}\right)
\end{aligned}
$$



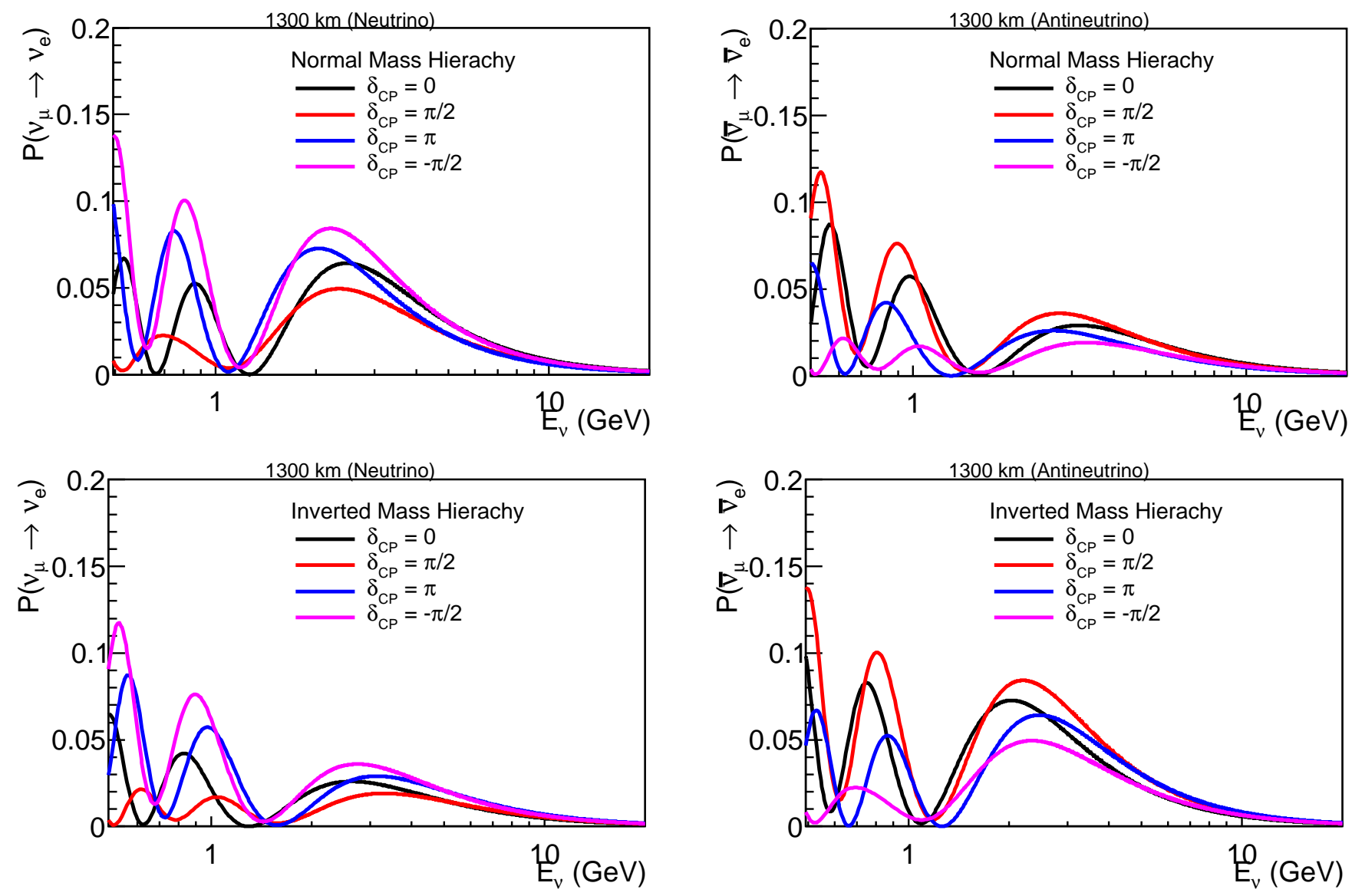

Figure 2: Electron neutrino and antineutrino appearance oscillation probabilities as a function of the neutrino energy $E_{\nu}$ at $L=1300 \mathrm{~km}$ for the indicated four values of the CP phase $\delta_{C P}$. The matter density $\rho$ and the electron fraction $Y_{e}$ are assumed to be $2.7 \mathrm{~g} / \mathrm{cm}^{3}$ and 0.5 , respectively. $\sin ^{2} \theta_{13}, \sin ^{2} \theta_{12}$, $\sin ^{2} \theta_{23}, \Delta m_{31}^{2}$, and $\Delta m_{21}^{2}$ are assumed to be $0.0219,0.304,0.5,2.4 \times 10^{-3} \mathrm{eV}^{2}$, and $7.65 \times 10^{-5} \mathrm{eV}^{2}$, respectively. Top (bottom) two panels correspond to the normal (inverted) mass hierarchy. Left (right) panels correspond to the neutrino (antineutrino) oscillation. In the normal (inverted) mass hierarchy, the neutrino (antineutrino) appearance are enhanced, and the antineutrino (neutrino) appearance are suppressed.

where $U_{\mu i}$ are the elements of the second row of the PMNS matrix and now the angle $\phi$ is $\sin \phi_{\mu \mu}=\frac{\left|U_{\mu 1}\right|^{2} \sin 2 \Delta_{21}}{\sqrt{\left|U_{\mu 1}\right|^{4}+\left|U_{\mu 2}\right|^{4}+2\left|U_{\mu 1}\right|^{2}\left|U_{\mu 2}\right|^{2} \cos 2 \Delta_{21}}}, \cos \phi_{\mu \mu}=\frac{\left|U_{\mu 1}\right|^{2} \cos 2 \Delta_{21}+\left|U_{\mu 2}\right|^{2}}{\sqrt{\left|U_{\mu 1}\right|^{4}+\left|U_{\mu 2}\right|^{4}+2\left|U_{\mu 1}\right|^{2}\left|U_{\mu 2}\right|^{2} \cos 2 \Delta_{21}}}$

By an accurate measurement of the survival probability and its $L / E_{\nu}$ dependence, combining with the accurate knowledge of at least some of the parameters involved, $\mathrm{MH}$ (the sign in front of $\phi$ ) can be determined. Detailed description of the issues involved will be given again in the next section.

Furthermore, there is a possibility of determining $\mathrm{MH}$ based on the observation of core-collapse supernovae. There are at best only a handful of the galactic core-collapse supernovae per century and their neutrino emission and detection is a complicated matter. On the other hand, neutrino sky coverage by the sufficiently large detectors has been on for about three decades already, and will continue in the foreseeable future. There is, therefore, a good chance that a high statistics SN neutrino sample will eventually become a reality and that the neutrino mass hierarchy can be deduced from its analysis. It is possible, perhaps even likely, that the SN neutrino spectra are not universal; they might depend on the progenitor mass, accretion or rotation. Nevertheless, some general features exist. Only recently it has 
been recognized that in the deep core region where the neutrino densities are very large the neutrino self-interaction could result in a hierarchy dependent collective flavor conversion. (For a review see Ref. [1:3]). Generally, this flavor conversion is not expected to affect the $\nu$ and $\bar{\nu}$ spectra in the case of $\mathrm{NH}$, but will alter significantly and differently the $\nu$ and $\bar{\nu}$ spectra in the case of IH. In addition, the neutrino spectra will be affected by the resonant matter effect in the outer lower mass density layers of the supernova. Resonance occurs for neutrinos in the case of $\mathrm{NH}$ and antineutrinos in the case of IH. Also, the detected signal could be possibly altered by the matter effect during the transitions through earth (for general references see the review in Ref.[14]). Given the fast development of the supernova explosion and neutrino production and propagation theory, we will not describe the possibilities of MH determination based on the future SN neutrino observation in further detail.
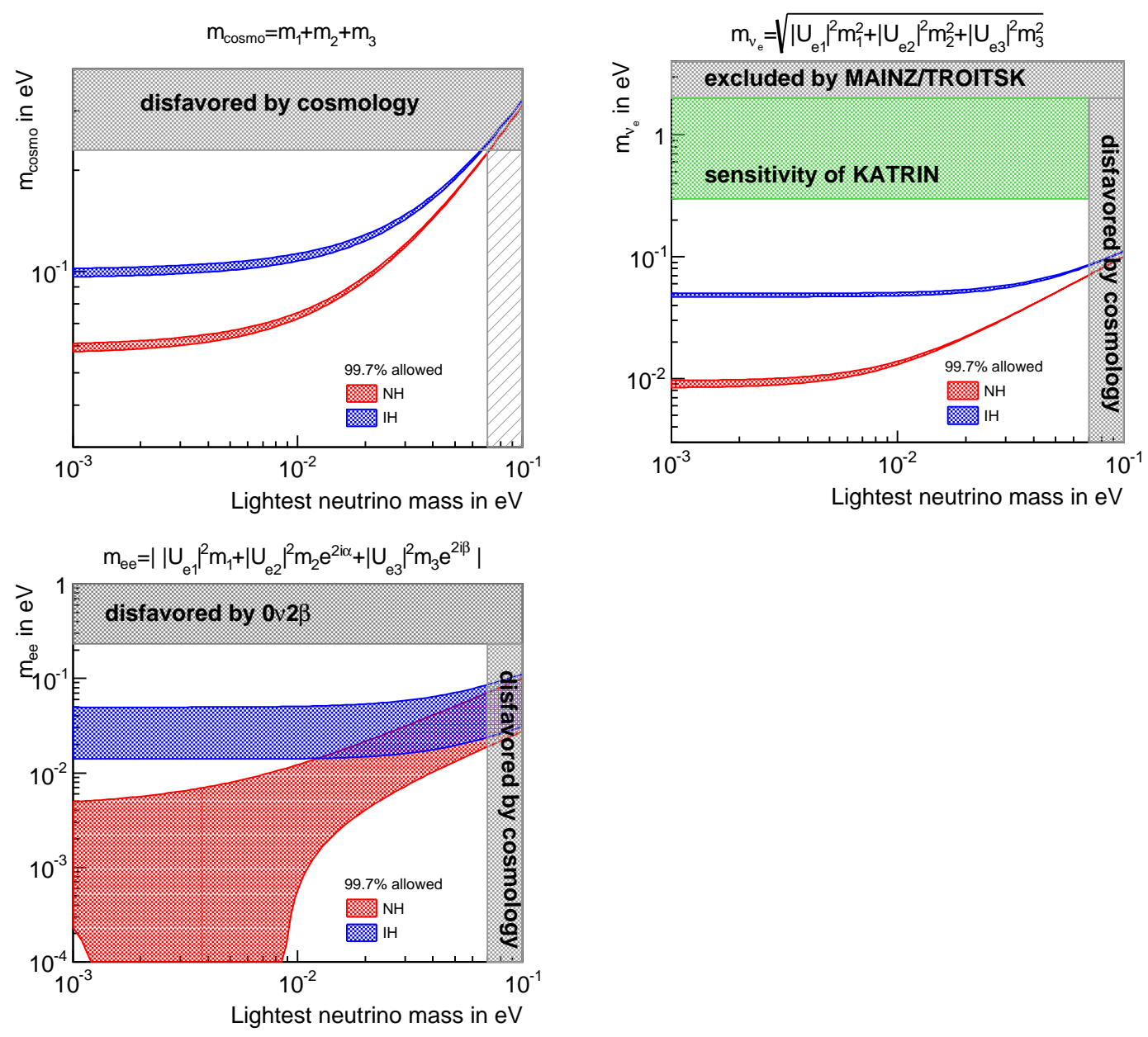

Figure 3: The top left, top right, and bottom left panels show the $m_{\text {cosmos }}$ (the summation of neutrino masses), $m_{\nu_{e}}$ (the effective neutrino mass probed in the beta decay experiments), and $m_{e e}$ (the effective neutrino mass probed in experiments searching for the neutrinoless double beta decay) vs. the lightest neutrino mass $m_{L}$ for both the normal and inverted mass hierarchy scenarios. The bands cover the allowed region given $3 \sigma$ neutrino mixing range in Table $\mathbb{W}$ and all possible values of Majorana phases.

Finally, some limited information on $\mathrm{MH}$ can come from cosmology, the study of the beta decay endpoint, and the study of the neutrinoless $\beta \beta$ decay. In cosmology, the sum of the neutrino masses $m_{\text {cosmos }}=\Sigma_{i} m_{i}$ (top left panel of Fig. 31)

$$
m_{\text {cosmos }}=\left\{\begin{array}{lr}
m_{L}+\sqrt{m_{L}^{2}+\Delta m_{21}^{2}}+\sqrt{m_{L}^{2}+\left|\Delta m_{31}^{2}\right|} \quad \text { (Normal Hierarchy) } \\
m_{L}+\sqrt{m_{L}^{2}+\left|\Delta m_{32}^{2}\right|}+\sqrt{m_{L}^{2}+\left|\Delta m_{31}^{2}\right|} \quad \text { (Inverted Hierarchy) }
\end{array}\right.
$$


could be determined. If that sum is below the minimum mass of $\sim 0.1 \mathrm{eV}$ corresponding to the inverted hierarchy, the existence of the normal hierarchy is indicated. Similarly, in the study of the beta decay spectrum near the endpoint, if the effective neutrino mass $m_{\nu_{e}}=\sqrt{\left|U_{e 1}\right|^{2} m_{1}^{2}+\left|U_{e 2}\right|^{2} m_{2}^{2}+\left|U_{e 3}\right|^{2} m_{3}^{2}}$ (top

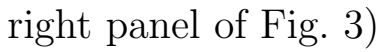

$$
m_{\nu_{e}}=\left\{\begin{array}{lr}
\sqrt{m_{L}^{2}+c_{13}^{2} s_{12}^{2} \Delta m_{21}^{2}+s_{13}^{2}\left|\Delta m_{31}^{2}\right|} & \text { (Normal Hierarchy) } \\
\sqrt{m_{L}^{2}+c_{13}^{2} c_{12}^{2}\left|\Delta m_{31}^{2}\right|+c_{13}^{2} s_{12}^{2}\left|\Delta m_{32}^{2}\right|} & \text { (Inverted Hierarchy) }
\end{array}\right.
$$

is measured to be smaller than $\sim 0.05 \mathrm{eV}$ corresponding to the minimum value of the inverted hierarchy, the existence of the normal hierarchy is suggested. In analogy, if the neutrinoless $\beta \beta$ decay is reliably observed and the deduced neutrino Majorana mass $m_{e e}=\left.|| U_{e 1}\right|^{2} m_{1}+\left|U_{e 2}\right|^{2} m_{2} e^{2 \alpha i}+\left|U_{e 3}\right|^{2} m_{3} e^{2 \beta i} \mid$

$m_{e e}^{2}= \begin{cases}\left(c_{12}^{2} c_{13}^{2} m_{L}+c_{13}^{2} s_{12}^{2} \sqrt{m_{L}^{2}+\Delta m_{21}^{2}} \cos 2 \alpha+s_{13}^{2} \sqrt{m_{L}^{2}+\left|\Delta m_{31}^{2}\right|} \cos 2 \beta\right)^{2}+ & \\ \left(c_{13}^{2} s_{12}^{2} \sqrt{m_{L}^{2}+\Delta m_{21}^{2}} \sin 2 \alpha+s_{13}^{2} \sqrt{m_{L}^{2}+\left|\Delta m_{31}^{2}\right|} \sin 2 \beta\right)^{2} & \text { (Normal Hierarchy) } \\ \left(c_{12}^{2} c_{13}^{2} \sqrt{m_{L}^{2}+\left|\Delta m_{31}^{2}\right|}+c_{13}^{2} s_{12}^{2} \sqrt{m_{L}^{2}+\left|\Delta m_{31}^{2}\right|} \cos 2 \alpha+s_{13}^{2} m_{L} \cos 2 \beta\right)^{2}+ & \\ \left(c_{13}^{2} s_{12}^{2} \sqrt{m_{L}^{2}+\left|\Delta m_{31}^{2}\right|} \sin 2 \alpha+s_{13}^{2} m_{L} \sin 2 \beta\right)^{2} & \text { (Inverted Hierarchy) }\end{cases}$

with $\alpha$ and $\beta$ being the Majorana phases (bottom left panel of Fig. B) is again below its minimal value corresponding to the inverted hierarchy, the normal hierarchy is implied. Note, however, that the proportionality between the double beta decay rate and $m_{e e}^{2}$ is only one, albeit the simplest, of the possible ways that the decay could occur.

The rest of this review is organized as follows. In the next section, the various experimental methods with sensitivity to the neutrino mass hierarchy are described in more detail. In Sec. B, the proposed experiments are described and discussed. Finally, in the last section the entire subject is briefly summarized.

\section{Various Methods to Determine the Mass Hierarchy}

Two known ways to determine the neutrino mass hierarchy (MH) were introduced in the previous section. The first one depends on the charge-current forward coherent scattering between $\nu_{e}$ (or $\bar{\nu}_{e}$ ) and the electrons in Earth (matter effect). The second one explores the small difference between $\Delta m_{31}^{2}$ and $\Delta m_{32}^{2}$. Both methods are now of practical significance due to the recently discovered non-zero, and relatively large, third neutrino mixing angle $\theta_{13}$. In this section, we describe the practical approaches to resolve $\mathrm{MH}$ that use available neutrino sources, namely i) the accelerator neutrinos, ii) the atmospheric neutrinos, and iii) the reactor antineutrinos. We also review the statistical interpretation of the mass hierarchy sensitivity, which is crucial for understanding the physics reach of various experiments to be discussed in the next section.

\subsection{The "Large" Third Neutrino Mixing Angle $\theta_{13}$}

In this section we briefly review the experimental discovery of the non-zero value of $\theta_{13} \approx 8.4^{\circ}$, which opens doors to $\mathrm{MH}$ determination.

Historically, the first attempt to determine the value of $\theta_{13}$ was done by the CHOOZ [15, 16] and Palo Verde [17] experiments in late 1990s and early 2000s. Both experiments were reactor antineutrino experiments searching for oscillations of $\bar{\nu}_{e}$ at baselines of $\sim 1 \mathrm{~km}$ with a single-detector configuration. No oscillations were observed in either experiment and an upper limit of $\sin ^{2} 2 \theta_{13}<0.12$ was set at $90 \%$ confidence level (C.L.) by CHOOZ. Almost 10 years later, in 2011, several hints suggested a non-zero $\theta_{13}$. The first one was based on a tension [18] between the KamLAND $\bar{\nu}_{e}$ disappearance measurement (a reactor antineutrino experiment at an average baseline of $\sim 180 \mathrm{~km}$ ) and the solar 
neutrino measurements (e.g. ratio of $\nu_{e}$ charge-current to $\nu_{e, \mu, \tau}$ neutral-current interactions from SNO). Subsequently, accelerator neutrino experiments MINOS [1.9] and T2K [20] reported on their searches of $\nu_{\mu}$ to $\nu_{e}$ appearance oscillation that is sensitive to $\theta_{13}$. In particular, T2K disfavored the $\theta_{13}=0$ hypothesis at $2.5 \sigma[20]$. In early 2012, the Double CHOOZ reactor antineutrino experiment reported that the $\theta_{13}=0$ hypothesis was disfavored at $1.6 \sigma$ with one detector [ [21] .

In March 2012, the Daya Bay reactor antineutrino experiment reported the discovery of non-zero $\theta_{13}$ with a $>5 \sigma$ significance [ [22]. About one month later, RENO confirmed the Daya Bay discovery with a $4.9 \sigma$ significance [2:3]. And later in that year, Daya Bay increased the significance to $7.7 \sigma$ with a larger data set [24]. By then, non-zero $\theta_{13}$ was firmly established. The left panel of Fig. 6 shows the current global status of $\sin ^{2} 2 \theta_{13}$ measurements compiled with the latest results from each experiment. The precision of this important parameter is still being improved by the current-generation experiments. In particular, the right panel of Fig. W shows the expected uncertainty of $\sin ^{2} 2 \theta_{13}$ from the Daya Bay experiment; by the end of the experiment it is expected to reach a better than $3 \%$ measurement precision of $\sin ^{2} 2 \theta_{13}$.
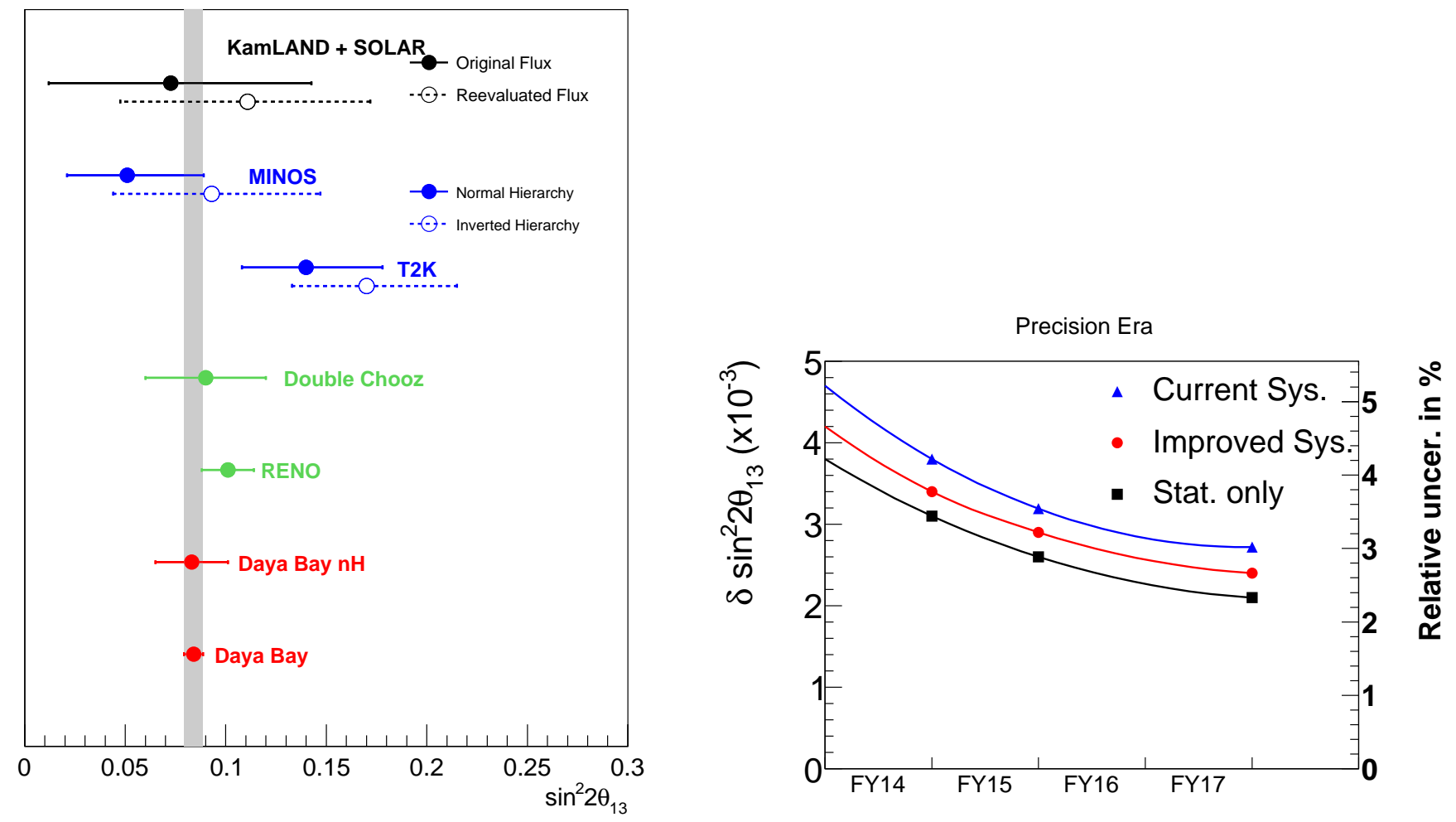

Figure 4: (Left) The current global status of the $\theta_{13}$ measurements. Results are taken from KamLAND+SOLAR [18], MINOS [25], T2K [26], Double CHOOZ [27], RENO [28], Daya Bay (nH) [29], and Daya Bay [30]. Among them, T2K and MINOS have assumed $\delta_{C P}=0$. (Right) Uncertainty on the Daya Bay measurement of $\sin ^{2} 2 \theta_{13}$ in the precision era (FY14-FY17, or 2015-2018) under different assumptions from Ref. [3]].

\subsection{Mass Hierarchy from Accelerator Neutrinos Appearance}

The accelerator neutrino experiments provided and continue to provide crucial inputs to the development of the 3-flavor PMNS model. Lederman, Schwartz, and Steinberger already in 1960's discovered the 
muon flavor neutrinos [32] at the Brookhaven National Laboratory (BNL) and showed that the lepton flavor appears to be conserved in the weak interaction. The tau flavor neutrinos $\nu_{\tau}$, the second to last elementary particle in the Standard Model experimentally confirmed, was discovered by the DONUT collaboration at the Fermi National Accelerator Laboratory (Fermilab) [33] in 2001. The K2K longbaseline experiment [34] confirmed the observation of the neutrino oscillation at energies comparable to those produced in the atmosphere. The OPERA experiment provided the evidence for $\nu_{\mu}$ to $\nu_{\tau}$ appearance [35]. The MINOS long-baseline experiment [36] provided the most precise measurement of the atmospheric mass-squared splitting $\Delta m_{32}^{2}$.

Very recently, the T2K experiment [26] discovered for the first time ever the neutrino appearance oscillation $\left(\nu_{e}\right.$ appearance from $\left.\nu_{\mu}\right)$, at more than $5 \sigma$ statistical significance, and confirmed the "large" non-zero $\theta_{13}$ discovered in the Daya Bay experiment [37]. The T2K experiment [26] together with the reactor neutrino experiments in Refs. [38, 39, 40] provide an initial interesting hint of the leptonic CP violation. The most precise measurement of the atmospheric mixing angle $\theta_{23}$ also comes from the T2K experiment. Besides the possible determination of $\mathrm{MH}$, the accelerator neutrino experiments are currently the only known reliable way to measure CP phase $\delta_{C P}$, as well as determining the $\theta_{23}$ octant: whether the $\nu_{3}$ contains more muon neutrino $\nu_{\mu}$ or tau neutrino $\nu_{\tau}$. Furthermore, the measurement of the third neutrino mixing angle $\theta_{13}$ from accelerator experiments together with the measurement of $\theta_{13}$ from reactor antineutrino experiments provides a nice unitarity test of the PMNS matrix [4T].

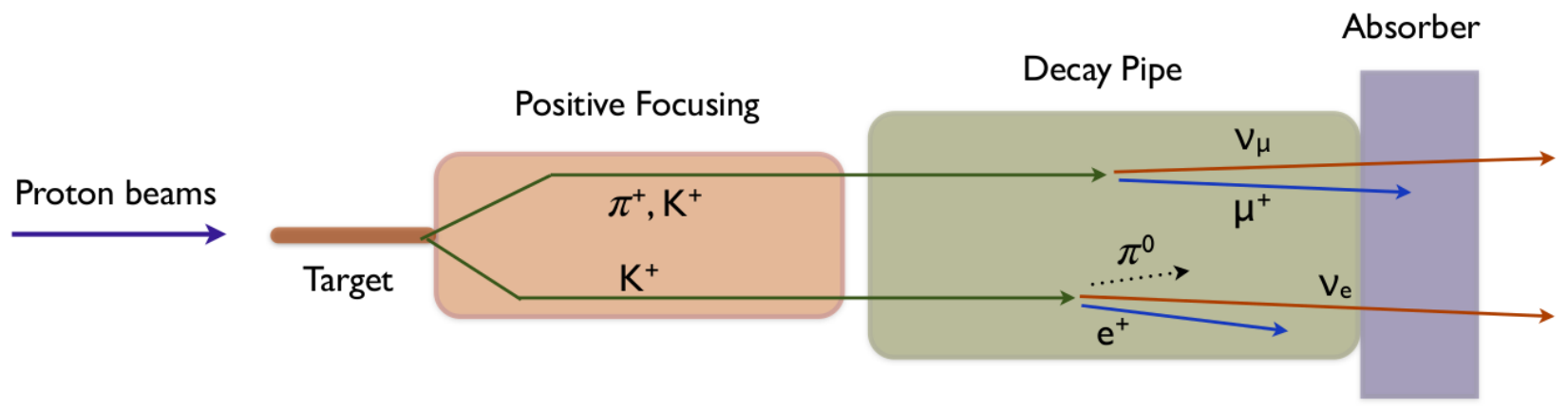

Figure 5: A schematic view of the conventional neutrino beamline. The magnetic field is toroidal and assumed to focus positive charged particle. The muon neutrinos and antineutrinos are mainly generated through the two-body decay of pions and kaons. The electron neutrinos and antineutrinos, one of the main backgrounds for the electron neutrino/antineutrino appearance measurement, are mainly generated through the three-body decay of kaons and muons. The dashed line illustrates the direction of very short-lived $\pi^{o}$.

Accelerator neutrinos are generated by a high-energy proton beam striking a nuclear target to produce pions and kaons, which in turn decay into neutrinos. A schematic view of a conventional neutrino beamline is shown in Fig. 15. First, energetic secondary pions and kaons are produced when high-energy proton beams interact with the nuclear target. Second, some of the charged pions and kaons within certain momentum range are focused by magnets (also known as horns), so that they are approximately traveling in parallel with the incident proton beam direction. The polarity of the magnet can be selected by changing the current in order to focus either the positive or negative charged particles. The charged pions and kaons then travel through a long decay pipe to provide enough time for them to decay. Finally, a thick absorber is placed at the end of decay pipe to absorb the muons (decay products of pions and kaons) and other remaining charged particles. The "on-axis" neutrino 
Booster Flux

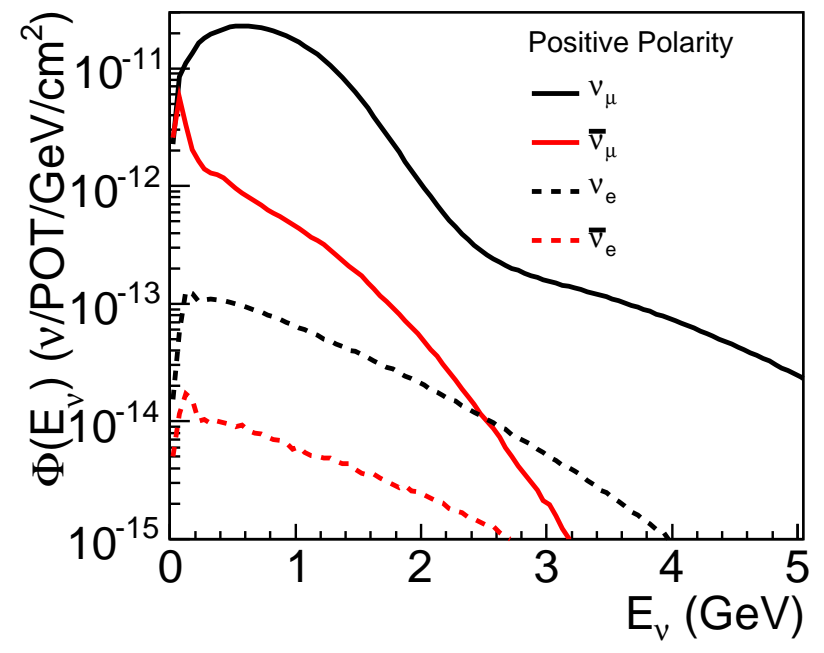

Booster Flux

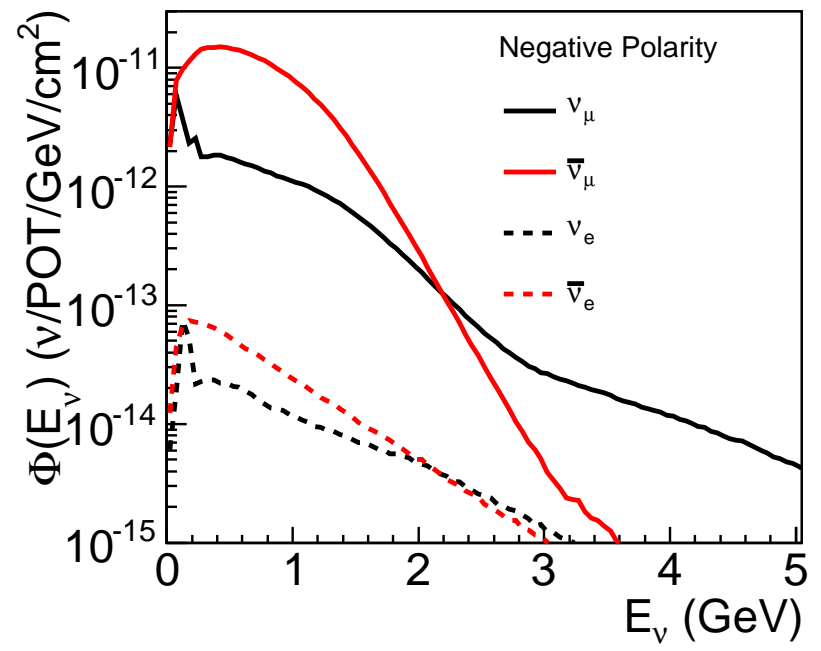

Figure 6: Neutrino fluxes for different species are shown for the Fermilab Booster neutrino beamline [43]. Left (right) panel represents the positive (negative) polarity which focuses positive (negative) charged particles in the magnetic horn. Besides the intrinsic $\nu_{e}$ and $\bar{\nu}_{e}$ fluxes, there are also sizable wrong-sign neutrinos $\left(\bar{\nu}_{\mu}\right.$ in the positive polarity mode and $\nu_{\mu}$ in the negative polarity mode), which are produced by the wrong-sign charged pions and kaons. Reproduced with the permission from Ref. [4:3].

beam refers to the case where the neutrino beam direction to detector is in parallel with the incident proton beam direction. In comparison, the "off-axis" neutrino beam refers to the case when there is a angle difference between these two directions. The "off-axis" beams pioneered by the E889 proposal at BNL [42] result in a narrower neutrino energy distribution.

The primary neutrino species are $\nu_{\mu}\left(\right.$ or $\left.\bar{\nu}_{\mu}\right)$ through positive (negative) charged pion and kaon 2-body decay:

$$
\begin{array}{rll}
\pi^{+} \rightarrow \mu^{+}+\nu_{\mu} & \left(\pi^{-} \rightarrow \mu^{-}+\bar{\nu}_{\mu}\right) & \text { B.R. } \sim 100 \% \\
K^{+} \rightarrow \mu^{+}+\nu_{\mu} & \left(K^{-} \rightarrow \mu^{-}+\bar{\nu}_{\mu}\right) & \text { B.R. } \sim 63 \%
\end{array}
$$

where B.R. stands for the branching ratio. Values are taken from Ref. [7]. There are also small amounts of irreducible $\nu_{e}$ and $\bar{\nu}_{e}$, mainly produced in the 3-body muon and kaon decays:

$$
\begin{array}{cll}
\mu^{+} \rightarrow e^{+}+\nu_{e}+\bar{\nu}_{\mu} & \mu^{-} \rightarrow e^{-}+\bar{\nu}_{e}+\nu_{\mu} & \text { B.R. } \sim 100 \% \\
K^{+} \rightarrow \pi^{o}+e^{+}+\nu_{e} & K^{-} \rightarrow \pi^{o}+e^{-}+\bar{\nu}_{e} & \text { B.R. } \sim 5 \% \\
K_{L}^{o} \rightarrow \pi^{+}+e^{-}+\bar{\nu}_{e} & K_{L}^{o} \rightarrow \pi^{-}+e^{+}+\nu_{e} & \text { B.R. } \sim 41 \% .
\end{array}
$$

These intrinsic $\nu_{e}$ and $\bar{\nu}_{e}$ in the beam are one of the main backgrounds for the $\nu_{\mu} \rightarrow \nu_{e}$ and $\bar{\nu}_{\mu} \rightarrow \bar{\nu}_{e}$ appearance measurements. As an example, Fig. [6 shows the neutrino fluxes at the Fermilab Booster neutrino beamline, where the incident beam proton kinetic energy is about $8 \mathrm{GeV}$.

The accelerator-produced neutrinos are generally detected through the charged-current interaction with nucleon/nuclei in the detector. In an oscillation experiment, there is typically a detector placed near the neutrino production region which is intended to measure the essentially unoscillated neutrino flux and a second detector placed far from the source to be sensitive to oscillations. The commonly used detector technologies for far detectors include water Cherenkov, liquid scintillator, magnetized iron plus scintillator, and liquid argon time projection chamber. The involved target nucleon/nuclei are typically ${ }^{1} \mathrm{H},{ }^{12} \mathrm{C},{ }^{16} \mathrm{O},{ }^{40} \mathrm{Ar}$, and ${ }^{56} \mathrm{Fe}$. 
Fig. $[$ shows the neutrino-nucleon and antineutrino-nucleon interaction cross sections. There are three main components based on the assumption that the neutrino interacts with one of the bound nucleons ("N") in the target.

In the quasi-elastic scattering (QE)

$$
\nu+" N " \rightarrow l^{-}+N^{\prime} \quad \bar{\nu}+" N " \rightarrow l^{+}+N^{\prime}
$$

the nucleon is converted into its isospin partner. In the resonance scattering (RES)

$$
\nu+" N " \rightarrow l^{-}+N^{*} \quad \bar{\nu}+" N^{\prime} \rightarrow l^{+}+N^{*}
$$

the nucleon is excited to a resonance state. And in the deep inelastic scattering (DIS)

$$
\nu+" q " \rightarrow l^{-}+X \quad \bar{\nu}+" q " \rightarrow l^{+}+X
$$

the neutrino is assumed to interact with valence or sea quarks ( " $q$ ") in the nucleon.

Besides these reaction mechanisms, neutrinos can also interact with more than one of the boundstate nucleons (i.e. meson-exchange currents and short-range correlations). In addition, neutrinos can also interact with the entire nucleus by the elastic scattering

$$
\nu+A \rightarrow l^{-}+A^{\prime} \quad \bar{\nu}+A \rightarrow l^{+}+A^{\prime}
$$

where the $\mathrm{A}^{\prime}$ is the daughter nucleus or by the coherent meson production

$$
\nu+A \rightarrow l^{-}+A+m^{+} \quad \bar{\nu}+A \rightarrow l^{+}+A+m^{-},
$$

where $m$ represents a meson (such as $\pi$ or $\rho$ ). The cross sections of the elastic and the coherent scattering in general are much smaller than the QE/RES/DIS cross sections.
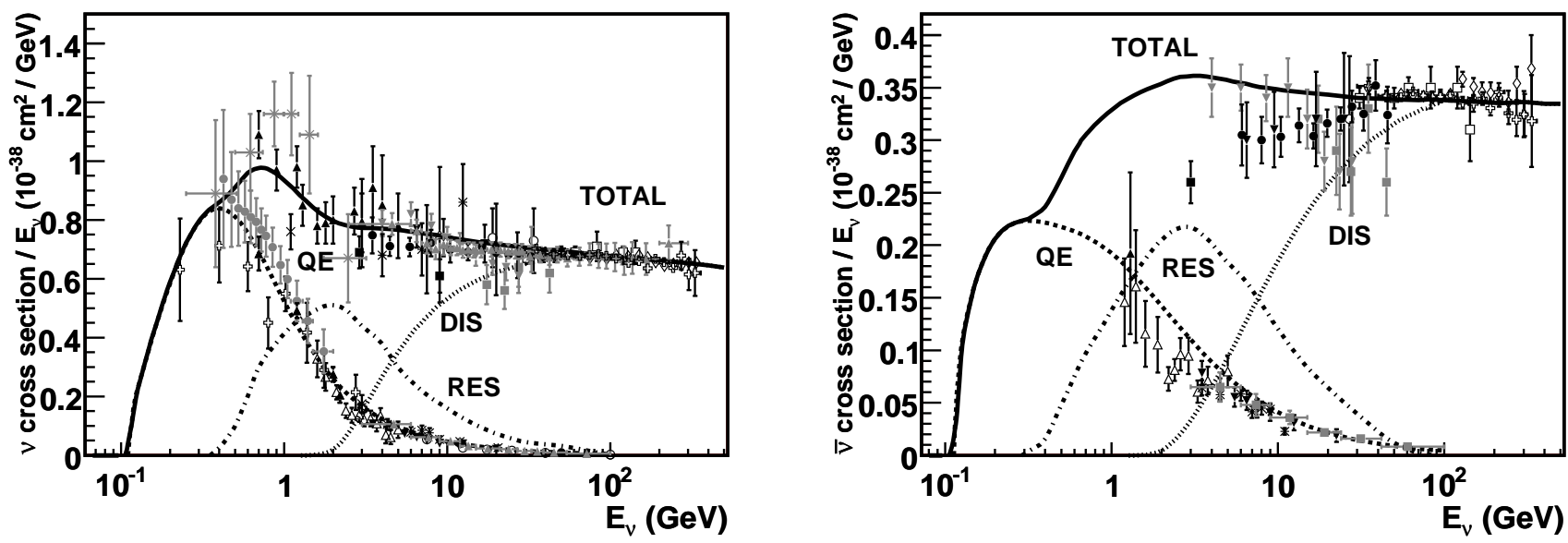

Figure 7: Summary of neutrino-nucleon interaction cross sections is reproduced with the permission from from Ref. [44]. Left (right) panel shows the ratio of neutrino (anti-neutrino) cross section with respect to the neutrino energy. Three main reaction mechanisms based on the assumption that the neutrino interacts with one of the bound nucleon ("N") in the target: quasi-elastic (QE), resonance (RES), and deep-inelastic scattering (DIS) are shown in addition to the total interaction cross section.

For neutrino oscillation measurements, the knowledge of the neutrino energy $E_{\nu}$ is crucial, as the neutrino oscillation probability is expressed as a function of $E_{\nu}$ and the neutrino traveling distance $L$. Depending on the reaction mechanism, there are different strategies to reconstruct the neutrino energy 
(see e.g. Ref. [44] and the references therein). For example, the quasi-elastic scattering is assumed to be a two body to two body scattering. For nucleons at rest in the lab frame, the neutrino energy can be reconstructed from the charged lepton kinematics (momentum and direction) together with the incident neutrino momentum direction. For the target nucleons bound in the nucleus, the neutrino energy reconstruction at QE kinematics $\nu_{l}+{ }^{\prime \prime} n^{\prime \prime} \rightarrow l+p$ can be written in this crude but simple and often used approximation as:

$$
E_{\nu}^{Q E}=\frac{2\left(M_{n}-E_{B}\right) E_{l}-\left[\left(M_{n}-E_{B}\right)^{2}+m_{l}^{2}-M_{p}^{2}\right]}{2\left[M_{n}-E_{B}-E_{l}+p_{l} \cos \left(\theta_{l}\right)\right]},
$$

with $M_{n}, M_{p}$, and $M_{l}$ are the mass of neutron, proton, and charged lepton, respectively. $E_{l}, p_{l}$, and $\theta_{l}$ are the energy, momentum, and polar angle of the scattered charged lepton. $E_{B}$ is the separation energy. The above method has a systematic bias, as the reaction mechanism should include the Fermi motion, involvement of more than one nucleon in the initial state (such as the short-range correlation), and final state interactions. The correct modeling of the reaction mechanism in the neutrino event generator is thus crucial. Another example is the deep inelastic scattering. The neutrino energy can be reconstructed by the calorimetry method, which sums the charged lepton energy with the energy of the hadronic system. In this method, the correct modeling of the hadronic energy response in the simulation and the correct estimation of the missing energy due to neutral particles (neutron and neutrino) becomes essential.

Fig. 8 shows the calculated $\nu_{\mu}$ to $\nu_{e}$ appearance oscillation probability vs. the ratio $L / E_{\nu}$. Due to the matter effect, the different $\mathrm{MH}$ leads to different behavior for the neutrino and antineutrino oscillations. In particular, at the first oscillation maximum $L / E_{\nu} \sim 510 \mathrm{~km} / \mathrm{GeV}$, the normal (inverted) MH would enhance the neutrino (antineutrino) oscillations, but suppress the antineutrino (neutrino) oscillations. Therefore, by precision measurements of the $\nu_{\mu}$ to $\nu_{e}$ and the $\bar{\nu}_{\mu}$ to $\bar{\nu}_{e}$ oscillations, MH can be determined. Comparing to Fig. [2, which is evaluated at fixed baseline $L=1300 \mathrm{~km}$, Fig. $\mathbb{Z}$ is made by scanning $L$ at fixed neutrino energy $E_{\nu}=2 \mathrm{GeV}$. Due to this choice, the matter effect is significant in the second and higher oscillation maxima in Fig. 8 . On the other hand, it is not as important for the second and higher oscillation maxima in Fig. 2 as the neutrino energy is much lower.

For neutrino oscillation experiments, the neutrino flavor at detection can be tagged by the charged lepton flavor. For the appearance experiments, the background determination is obviously essential. Besides the intrinsic $\nu_{e}$ and $\bar{\nu}_{e}$ beam backgrounds, there are two additional backgrounds. The first one is the neutral-current $\pi^{o}$ production

$$
\nu+" N " \rightarrow \nu+\pi^{o}+X
$$

Due to its very short lifetime $\left(\sim 8.5 \times 10^{-17} \mathrm{~s}\right.$ in its at-rest frame $), \pi^{o}$ would immediately decay after production. About $99 \%$ of $\pi^{o}$ would decay into two gammas. If one of the gammas is missed in the reconstruction (if it has low energy or because it is too close to the other gamma), the $\pi^{o}$ could be misidentified as an $e^{ \pm}$event. The second one is the charged-current $\nu_{\tau}$ scattering, when the neutrino energy is high enough to produce a $\tau$ lepton,

$$
\nu_{\tau}+" N " \rightarrow \tau^{-}+X \quad \bar{\nu}_{\tau}+" N " \rightarrow \tau^{+}+X
$$

About $18 \%$ of $\tau^{ \pm}$would decay through

$$
\tau^{-} \rightarrow e^{-}+\bar{\nu}_{e}+\nu_{\tau} \quad \tau^{+} \rightarrow e^{+}+\nu_{e}+\bar{\nu}_{\tau}
$$

in which the $\mathrm{e}^{ \pm}$could mimic the $\nu_{e} / \bar{\nu}_{e}$ charged-current interaction. 

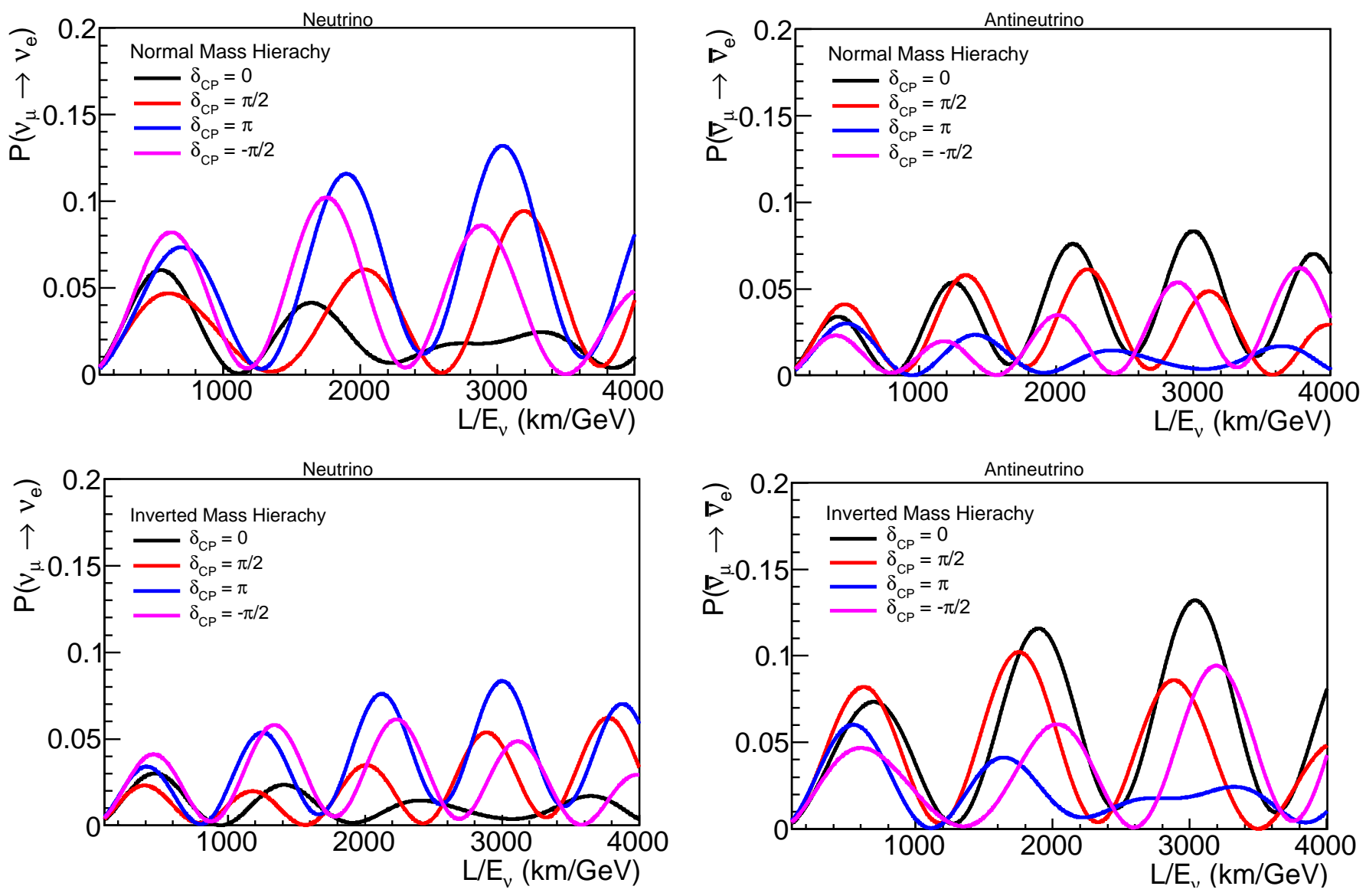

Figure 8: The $\nu_{\mu}$ to $\nu_{e}$ appearance oscillation probabilities are shown vs. the ratio $L / E_{\nu}$. The matter density $\rho$ and the electron fraction $Y_{e}$ are assumed to be $2.7 \mathrm{~g} / \mathrm{cm}^{3}$ and 0.5 , respectively. $\sin ^{2} \theta_{13}$, $\sin ^{2} \theta_{12}, \sin ^{2} \theta_{23}, \Delta m_{31}^{2}$, and $\Delta m_{21}^{2}$ are assumed to be $0.0219,0.304,0.5,2.4 \times 10^{-3} \mathrm{eV}^{2}$, and $7.65 \times 10^{-5}$ $\mathrm{eV}^{2}$, respectively. Top (bottom) panels correspond to the normal (inverted) mass hierarchy. Left (right) panels correspond to the neutrino (antineutrino) oscillation. The plot is made by scanning distance $L$ at a fixed neutrino energy $E_{\nu}=2 \mathrm{GeV}$. Different curves represent different values of the CP phase $\delta_{C P}$.

\subsection{Mass Hierarchy from Atmospheric neutrinos}

In 1998, the Super Kamiokande collaboration reported a zenith angle dependent deficit of atmospheric muon neutrinos, inconsistent with the expected atmospheric neutrino flux [45]. This seminal result pioneered the entire field of the neutrino flavor oscillations. Now, with the next-generation gigantic detectors, atmospheric neutrinos provide a new opportunity to determine $\mathrm{MH}$.

Atmospheric neutrinos are generated by high-energy cosmic rays colliding with nuclei in the upper atmosphere. The collision produces a shower of hadrons dominated by pions with a small amount of kaons. Similarly to the accelerator neutrinos, atmospheric neutrinos are generated through the decay of pions, muons, and kaons. The positively charged pions decay to a positively charged muon and a muon neutrino. The positively charged muons further decay to a positron, an electron neutrino, and a muon antineutrino. The decay chain of negatively charged pions is analogous. Therefore, one naively expects a two-to-one ratio of muon neutrinos to electron neutrinos, when the momentum distribution

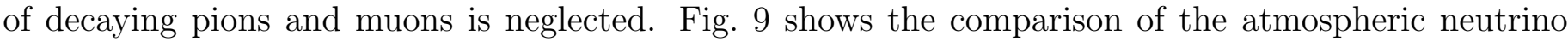
flux calculations for the Kamioka site for different neutrino flavors, where above $2 \mathrm{GeV}$ neutrino energy the ratio of muon neutrino to the electron neutrino begins to increase. 

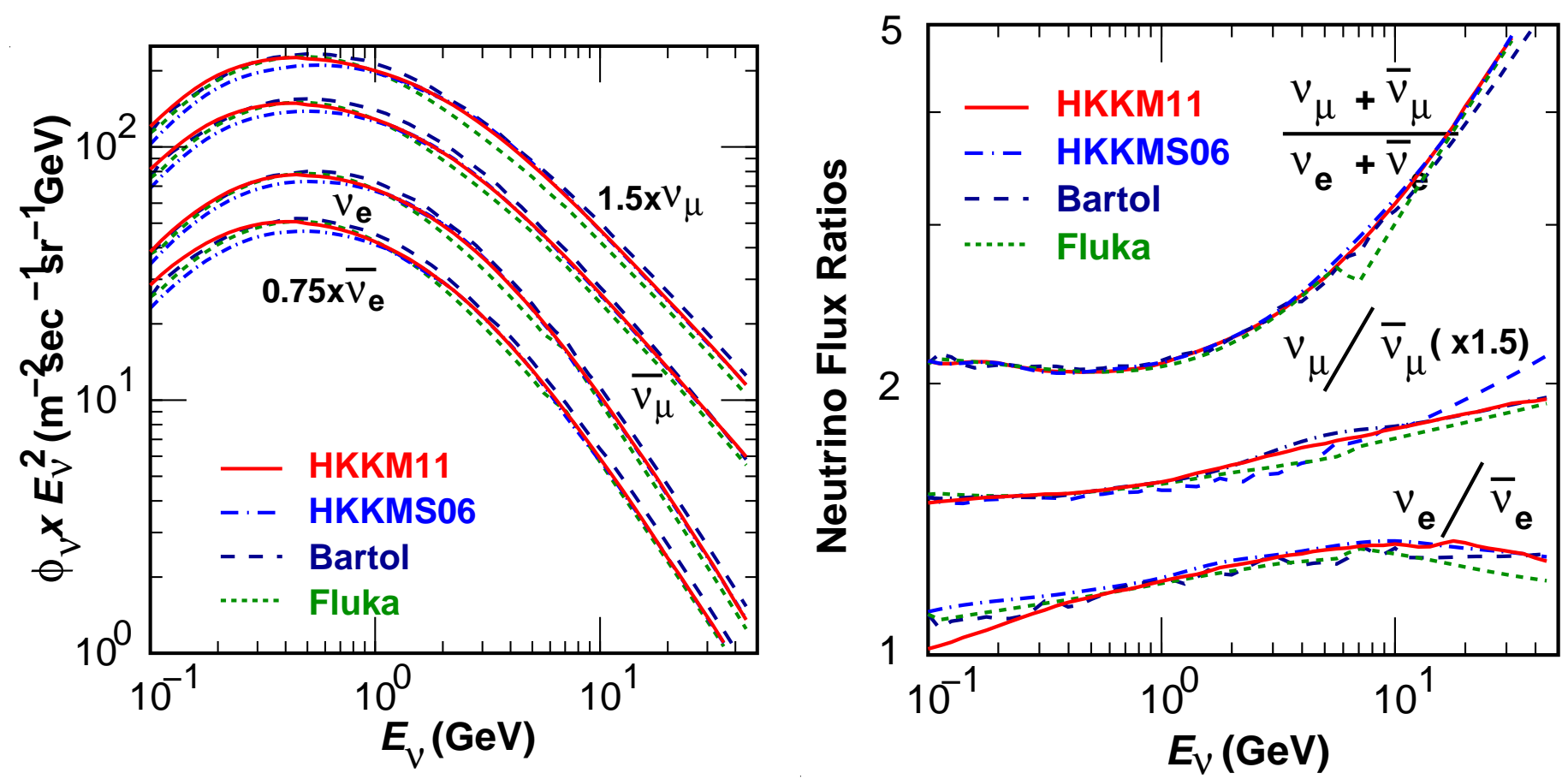

Figure 9: Atmospheric neutrino flux prediction is reproduced with the permission from Ref. [46]. The left panel shows the atmospheric neutrino fluxes calculated for the Kamioka site averaged over all directions. The right panel shows the corresponding flux ratios. Four models, HKKM11 [46], HKKMS06 [47], Bartol [48], and Fluka [4.9] are compared.

In addition, the ratios of neutrinos to antineutrinos are larger than unity. This is because the positively charged pions, containing the $u$ and $\bar{d}$ quarks, are expected to have a higher production yield than the the negatively charged pions containing the $d$ and $\bar{u}$ quarks. This, in turn, is caused by the fact that the primary cosmic rays are typically the high-energy protons, containing two $u$ and one $d$ valence quarks.

The zenith angle of the atmospheric neutrinos is directly correlated with the neutrino traveling distance for a detector near the earth surface as shown in the left panel of Fig. ए0. The neutrino detection relies on the charged-current neutrino-nuclei interaction as discussed in the previous section. In particular, as shown in Fig. 7 , the neutrino interaction cross section is much larger (about factor of 2 at high energies) than the antineutrino interaction cross section.

Similarly to the accelerator neutrino case, $\mathrm{MH}$ can be determined through the matter effect as elaborated in Ref. [50, 5], 52, 5:3, 54] among others. It is necessary, however, to take into account that the earth matter density is not constant, as shown in the right panel of Fig. $\mathbf{0}$.

Figs. 10 and 12 show the calculated oscillation probability for the atmospheric muon neutrinos and electron neutrinos, respectively. In NH scenario, we have the following features

- Muon neutrino disappearance $\nu_{\mu} \rightarrow \nu_{\mu}$ and muon antineutrino disappearance $\bar{\nu}_{\mu} \rightarrow \bar{\nu}_{\mu}$ : there is a resonance for the neutrinos at energy $E_{\nu} \sim 5 \mathrm{GeV}$ and zenith angle $\cos \theta \sim-0.95$ (neutrinos traveling from the other side of the earth) compared to the antineutrinos.

- Muon neutrino to electron neutrino appearance $\nu_{\mu} \rightarrow \nu_{e}$ and muon antineutrino to electron antineutrino appearance $\bar{\nu}_{\mu} \rightarrow \bar{\nu}_{e}$ : there is a much larger oscillation in the neutrinos than in the antineutrinos.

- Electron neutrino disappearance $\nu_{e} \rightarrow \nu_{e}$ and electron antineutrino disappearance $\bar{\nu}_{e} \rightarrow \bar{\nu}_{e}$ : there is a much larger oscillation in the neutrinos than in the antineutrinos. 
- Electron neutrino to muon neutrino appearance $\nu_{e} \rightarrow \nu_{\mu}$ and electron antineutrno to muon antineutrino appearance $\bar{\nu}_{e} \rightarrow \bar{\nu}_{\mu}$ : there is a much larger oscillation in the neutrinos than in the antineutrinos.

In IH scenario, the behavior of neutrino oscillations are exchanged with those of antineutrino oscillations.

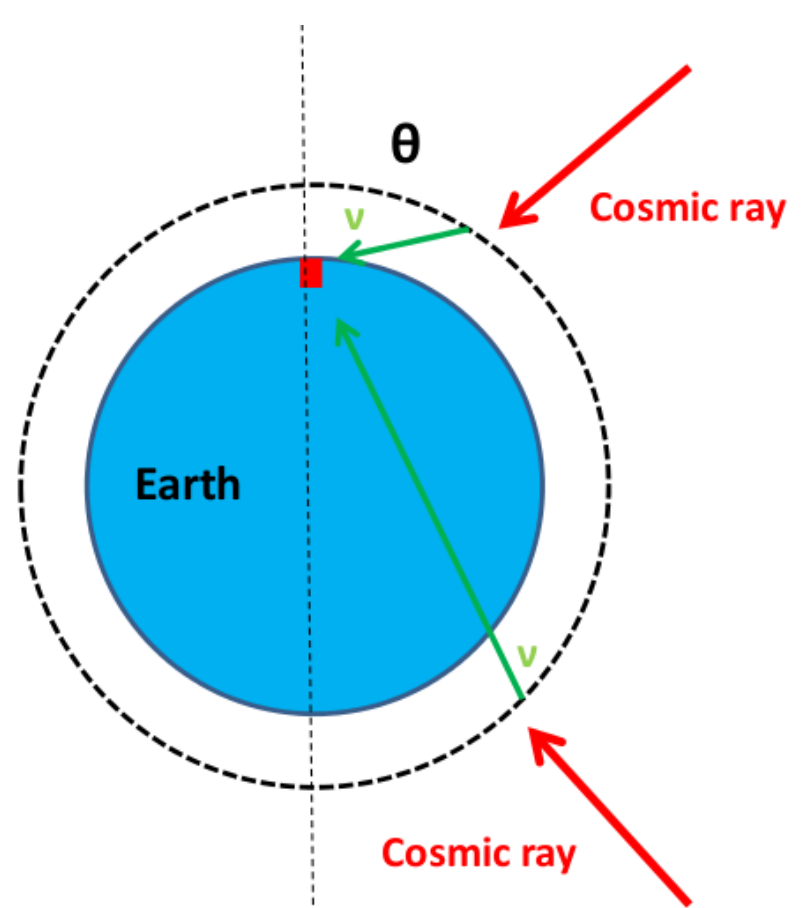

(a)

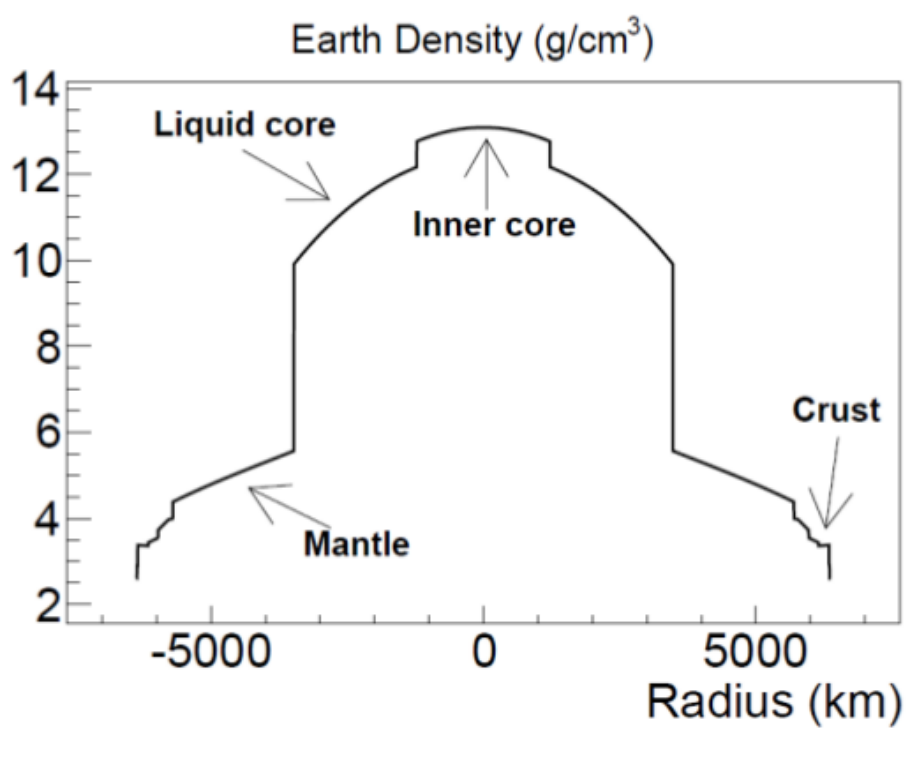

(b)

Figure 10: Left panel: a schematic view of the atmospheric neutrinos generation and detection. The zenith angle $\theta$ of the neutrinos are indicated. Right panel: The matter density of the Earth is shown as a function of the radius. The data is based on the Preliminary Reference Earth Model (PREM) [55].

The atmospheric neutrino flux contains four neutrino species $\left(\nu_{\mu}, \bar{\nu}_{\mu}, \nu_{e}, \bar{\nu}_{e}\right)$ and the detectors are usually only sensitive to the neutrino flavor. Therefore, one cannot disentangle the muon neutrino disappearance from the electron neutrino to muon neutrino appearance. Similarly, one cannot disentangle the electron neutrino disappearance from the muon neutrino to electron neutrino appearance. Nevertheless, they can still be sensitive to the matter effect, as the modification of neutrino oscillations due to the matter effect is large. In addition, as the neutrino flux is higher than the antineutrino flux and the neutrino-nuclei cross section is larger than the antineutrino-nuclei cross section, one could determine $\mathrm{MH}$ with a detector that is not sensitive to the lepton (in practice muon) charge. The sensitivity to $\mathrm{MH}$ can be further enhanced with a magnetized detector which is sensitive to the muon charge. 

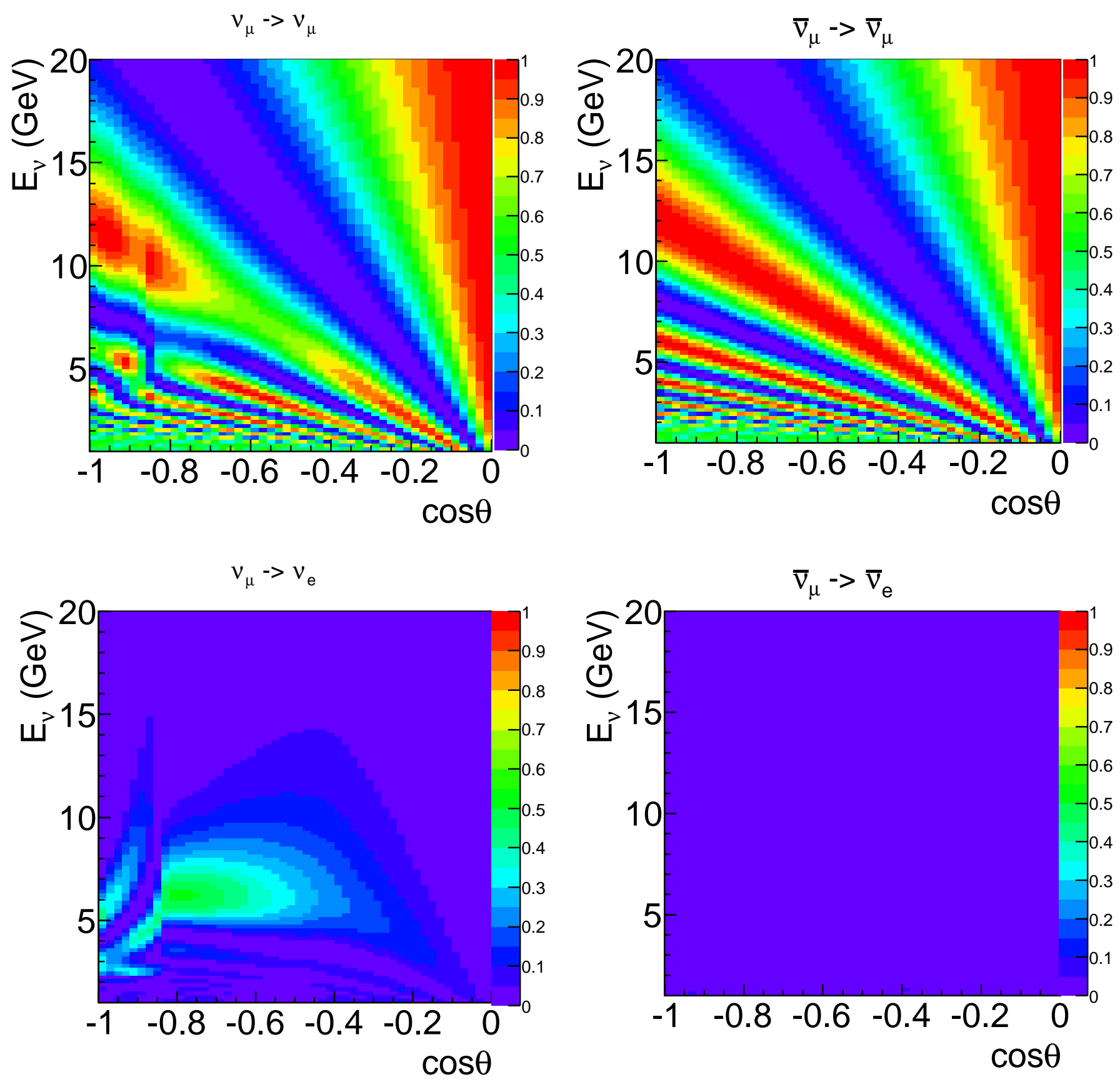

Figure 11: Atmospheric muon neutrino oscillations calculated with the nuosc package [56] are shown as a function of the zenith angle $\cos \theta$ and neutrino energy $E_{\nu}$. Top (bottom) panels show the muon neutrino disappearance (electron neutrino appearance). Left (right) panels show the neutrino (antineutrino) oscillations. The normal mass hierarchy and zero CP phase $\delta_{C P}=0$ is assumed. For the inverted mass hierarchy, the neutrino and antineutrino oscillation patterns are exchanged. 

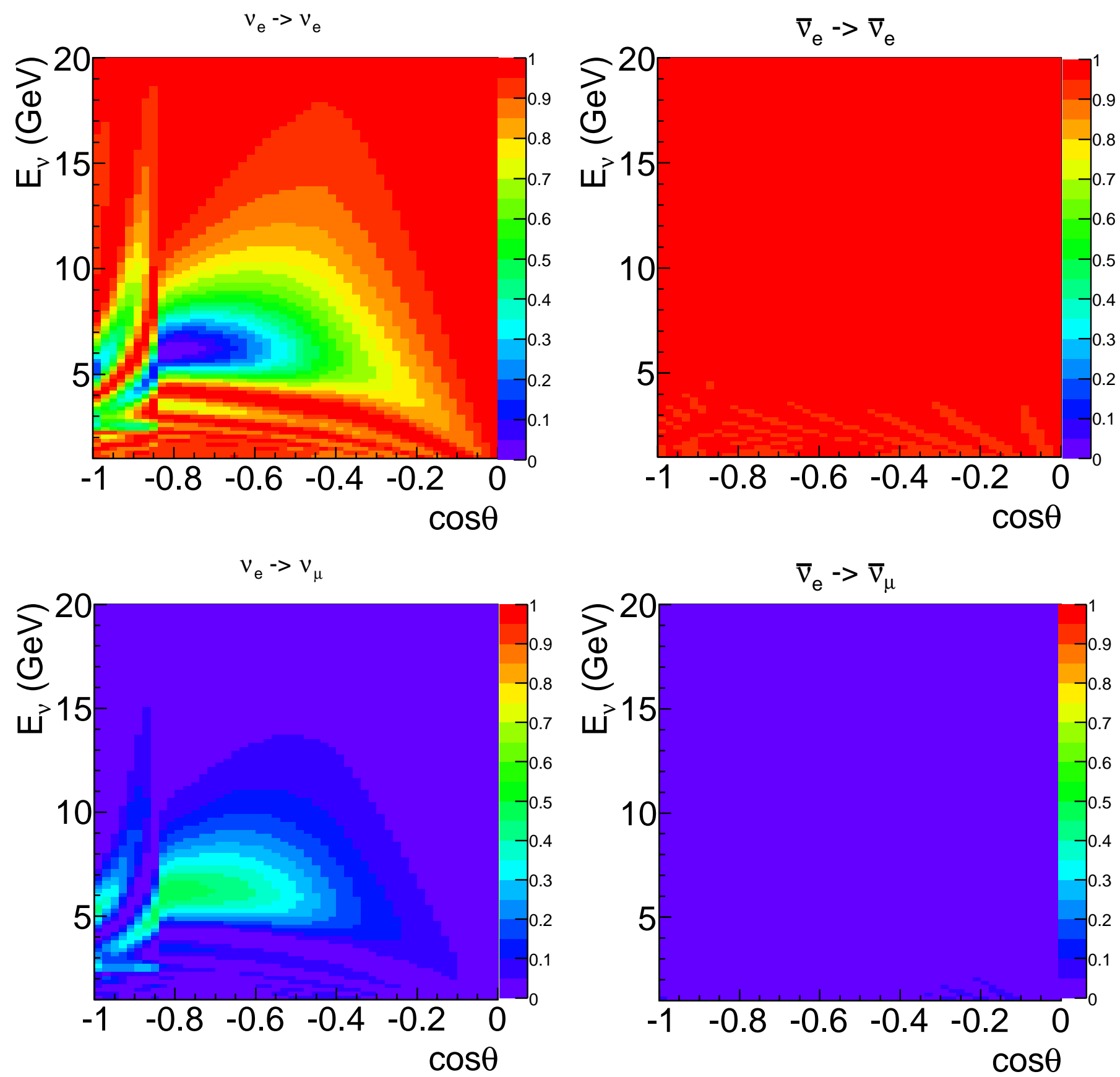

Figure 12: Atmospheric electron neutrino oscillations calculated with the nuosc package [56] are shown as a function of the zenith angle $\cos \theta$ and neutrino energy $E_{\nu}$. Top (bottom) panels show the electron neutrino disappearance (muon neutrino appearance). Left (right) panels show the neutrino (antineutrino) oscillations. The normal mass hierarchy and zero CP phase $\delta_{C P}=0$ is assumed. For the inverted mass hierarchy, the neutrino and antineutrino oscillation patterns are exchanged.

\subsection{Mass Hierarchy from Reactor Antineutrinos}

Reactor antineutrino experiments have a glorious history. In 1956, Cowan and Reines first experimentally discovered neutrinos at the Savannah River reactor power plant in the U.S. [57]. In 2005, the KamLAND experiment in Japan observed the neutrino oscillations in the solar sector [58]. Recently, the Daya Bay experiment in China, the RENO experiment in Korea, and the Double-Chooz experiment 
in France reported the discovery of non-zero $\theta_{13}$, the third neutrino mixing angle in 2012 [22, 2:3, 21]]. In the future, besides the determination of the mass hierarchy, the reactor neutrino experiments will also provide precision measurements of neutrino mixing parameters including the mixing angles $\theta_{13}$ and $\theta_{12}$ and the mass-squared splittings $\Delta m_{32}^{2}$ and $\Delta m_{21}^{2}$.

Nuclear power reactors produce electricity by the sustained nuclear chain reaction, and are essentially pure electron antineutrino $\bar{\nu}_{e}$ sources. In every nuclear fission $\sim 200 \mathrm{MeV}$ energy is released together with on average six $\bar{\nu}_{e}$ emitted by the $\beta$-decay of the fission fragments. ${ }^{\mathbb{1}}$ For each 1 gigawatt (GW) of the reactor thermal power, about $2 \times 10^{20} \bar{\nu}_{e}$ are emitted isotropically every second, making nuclear reactors one of the most powerful man-made neutrino sources. ${ }^{235} \mathrm{U}$ and ${ }^{238} \mathrm{U}$, together with the ${ }^{239} \mathrm{Pu}$ and ${ }^{242} \mathrm{Pu}$ produced by breeding in the reactor, are the main sources of the reactor $\bar{\nu}_{e}$. The corresponding $\bar{\nu}_{e}$ energy spectra are shown in Fig. 3].

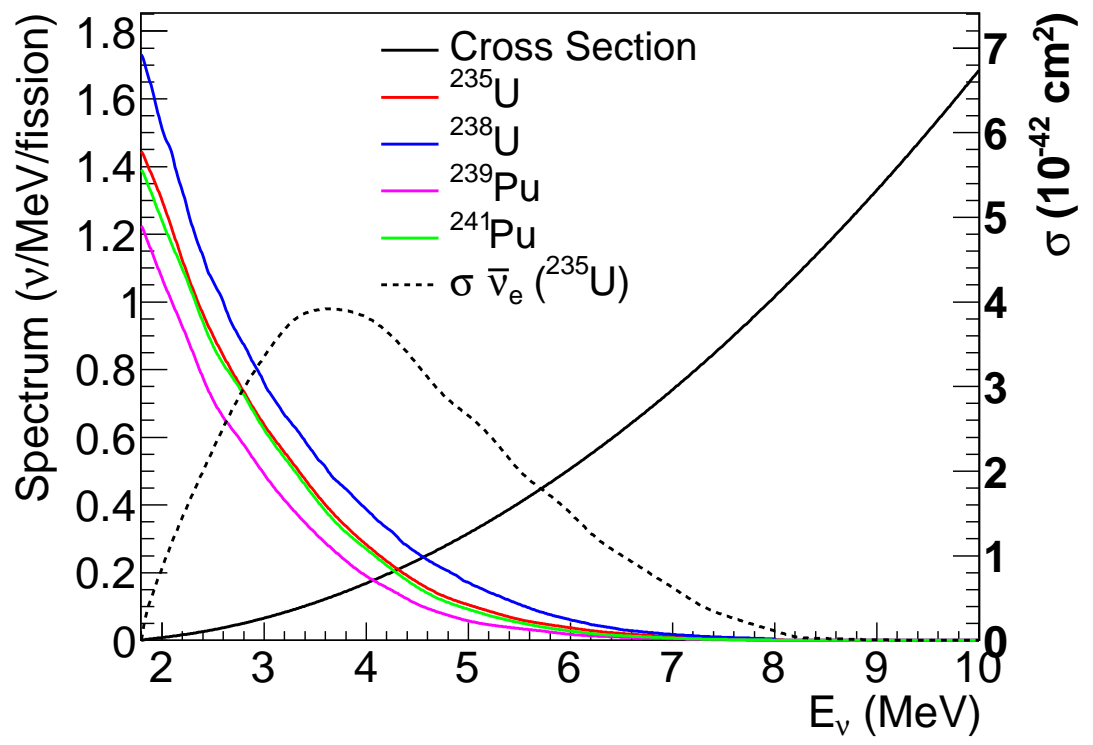

Figure 13: $\quad \bar{\nu}_{e}$ energy spectra (four curves with negative slopes) for ${ }^{235} \mathrm{U}\left[[5.9],{ }^{238} \mathrm{U}[6 \mathrm{60}],{ }^{239} \mathrm{Pu}[\mathrm{61}]\right.$, and ${ }^{241} \mathrm{Pu}[6 \mathrm{I}]$ are shown. The curve with the positive slope represents the cross section of the inverse beta decay (IBD) process. The convoluted IBD spectrum, measured in experiments, is shown as the dotted line.

The primary method to detect reactor $\bar{\nu}_{e}$ is using the inverse beta decay (IBD) reaction $\bar{\nu}_{e}+p \rightarrow e^{+}+n$ in which electron antineutrino interacts with a free proton. The final state particles are a positron and a neutron. An IBD event is represented by a coincident signal consisting of i) a prompt signal induced by the positron ionization and annihilation inside the detector such as a liquid scintillator (LS) detector and ii) a delayed signal produced by the neutron capture on a proton or a nucleus (such as Gd) with a large neutron capture cross section. In particular, the neutron capture on Gd releases multiple gammas with a total energy $\sim 8 \mathrm{MeV}$. With $0.1 \% \mathrm{Gd}$-doped LS, the average time between the prompt and the delayed signal is about $30 \mu \mathrm{s}$. In comparison, for neutron capture on hydrogen a single gamma with energy of $\sim 2.2 \mathrm{MeV}$ is emitted. For liquid scintillator $C_{n} H_{\sim 2 n}$, the average time between the prompt and the delayed signal is about $200 \mu \mathrm{s}$.

Fig. 14 illustrates the reaction and detection principle of the IBD process. The cross section of the IBD process is shown as the solid black line in Fig. 13 with units showing on the right axis. The convoluted energy spectrum, i.e. the product of the reactor antineutrino flux and the IBD cross

\footnotetext{
${ }^{1}$ There is also a small component of the electron neutrinos $\nu_{e}$ with energy $\sim 0.1 \mathrm{MeV}$ from the neutron activation of the shielding materials.
} 
section, is plotted as the dashed line. It begins at the threshold of $\sim 1.8 \mathrm{MeV}$ neutrino energy $E_{\nu}$, and peaks around $4 \mathrm{MeV}$. The tail of the spectrum extends beyond $8 \mathrm{MeV}$. Due to the time and space correlations, IBD can be easily distinguished from radioactive backgrounds which mostly consist of only a single signal. Furthermore, the energy of the prompt signal (also known as the visible energy $E_{v i s}$ ) is directly related to the neutrino energy:

$$
E_{\nu}=E_{e^{+}}+m_{n}+T_{n}-m_{p} \approx E_{\text {prompt }}+0.78 \mathrm{MeV}
$$

where $m_{e}, m_{n}$, and $m_{p}$ are the mass of electron, neutron, and proton, respectively. $T_{n}$ is the relatively small $\left(\sim\right.$ a few keV) kinetic energy of the recoiled neutron. Besides the energy of the positron, $E_{\text {prompt }}$ also contains the mass of electron that annihilates with the positron. The straight-forward method of reconstructing the neutrino energy is a very attractive feature for the study of neutrino oscillations that require good knowledge of the neutrino energy.

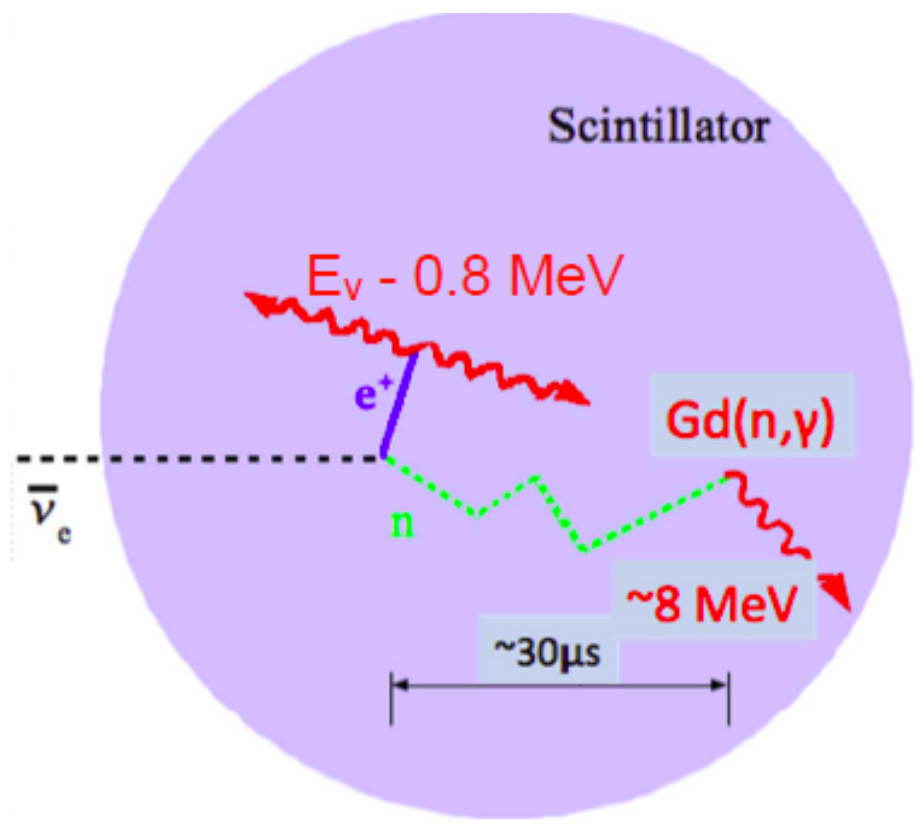

Figure 14: The detecting principle of IBD is shown. This figure is reproduced with the permission from Ref. [62].

The basis for the $\mathrm{MH}$ determination using the $\bar{\nu}_{e}$ disappearance has been extensively discussed in Refs. [63, 64, 65, 66, 67, 68, 69, 70, 77, 72, 73], and is illustrated in Eq. ([2) in Sec. (1. In order to further elaborate on this point, we define the effective mass-squared splitting as:

$$
\Delta m_{\text {eff }}^{2}=\frac{4 E_{\nu}}{L} \cdot\left(2 \Delta_{32} \pm \phi_{e e}\right)= \begin{cases}2 \Delta m_{32}^{2}+\Delta m_{\phi}^{2} & \text { (Normal Hierarchy) } \\ 2 \Delta m_{32}^{2}-\Delta m_{\phi}^{2} & \text { (Inverted Hierarchy) }\end{cases}
$$

which corresponds to the fourth term in Eq. (ए2). Here, we define $\Delta m_{\phi}^{2}:=4 \cdot \phi_{e e} \cdot E_{\nu} / L$.

In the left panel of Fig. [5, the $\Delta m_{\phi}^{2}$ is plotted as a function of the visible energy $E_{\text {vis }} \equiv E_{\text {prompt }}$ as well as the baseline $L$. At a baseline $L \sim 60 \mathrm{~km}, \Delta m_{\phi}^{2}$ has a clear energy dependence. In particular, the $\Delta m_{\phi}^{2}$ at low energy (2-4 MeV) is larger than that at high energy (4-8 MeV), providing an opportunity to determine MH. For the normal (inverted) MH, the $\Delta m_{\text {eff }}^{2}$ measured at low energy (2-4 MeV) would be higher (lower) than that measured at high energy (4-8 MeV). However, in order to perform such a measurement successfully, the energy resolution of the detector must be very good (better than $1.9 \%$ for $\delta E / E$ at $2.5 \mathrm{MeV}$ ), since the oscillations corresponding to $\Delta m_{\text {eff }}^{2}$ are very fast at low energy and a cruder energy resolution would make the oscillation pattern to disappear. On the other hand, at short 
baseline $(L<20 \mathrm{~km}), \Delta m_{\phi}^{2}$ is essentially a constant, thus it would be impossible to determine $\mathrm{MH}$ through comparing $\Delta m_{e f f}^{2}$ values at low and high energy regions. The purple line in the left panel of Fig. 15 represents the approximate boundary of degenerate mass-squared difference. The right side of the purple line alone will yield negligible contributions to $\mathrm{MH}$.

Alternatively, if one could measure $\Delta m_{\text {eff }}^{2}$ very precisely in both the electron antineutrino disappearance channel and in the muon neutrino and antineutrino disappearance channels, one can be sensitive to $\mathrm{MH}[\mathbf{7 4}, 65]$. This is because the $\Delta m_{\phi}^{2}$ values in these two channels are different. More specifically, as shown in the right panel of Fig. 15, $\Delta m_{\phi}^{2}$ ee is larger than $\Delta m_{\phi \mu \mu}^{2}$. Therefore, we have

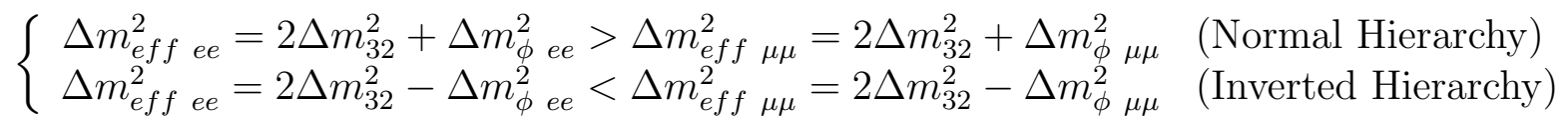

Since the difference between the $\Delta m_{\phi}^{2}$ in these two channels is quite small $\left(\sim 5 \times 10^{-5} \mathrm{eV}^{2}\right)$, the required precision of $\Delta m_{\text {eff }}^{2}$ in both channels would be about $\sim 1 \times 10^{-5} \mathrm{eV}^{2}$, which is very challenging. Therefore, this method is likely to serve as a validation once the $\mathrm{MH}$ is already determined by other means.
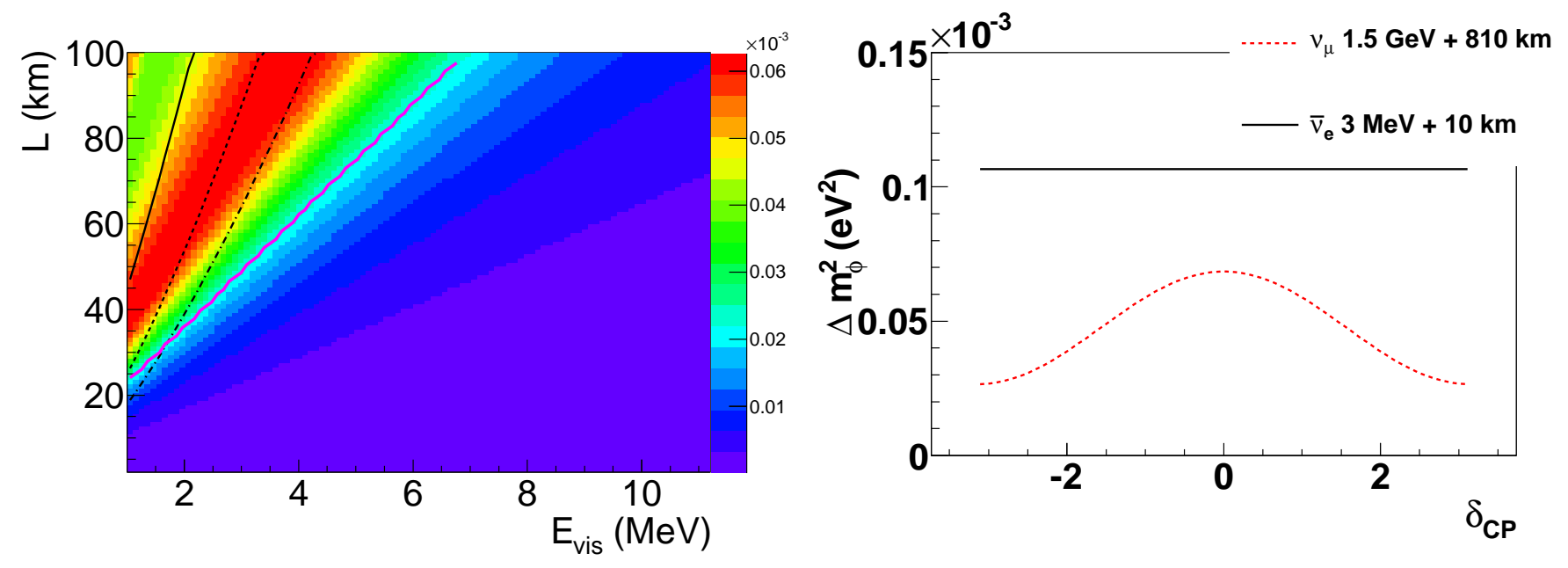

Figure 15: (Left) The effective mass-squared difference $\Delta m_{\phi}^{2}$, as a function of baseline (y-axis) and visible energy $E_{\text {vis }} \simeq E_{\nu}-0.8 \mathrm{MeV}$ (x-axis). The color represents the size of $\Delta m_{\phi}^{2}$ in $\mathrm{eV}^{2}$. The solid, dashed, and dotted lines are three choices of detector energy resolution with $2.8 \%, 5.0 \%$, and $7.0 \%$ at 1 $\mathrm{MeV}$, respectively. The left side of these lines (low values of $E_{\mathrm{vis}}$ ) will yield negligible contributions to the differentiation of the $\mathrm{MH}$ due to large values of $L / E_{\nu}$ The purple solid line represents the approximate boundary of the degenerate mass-squared difference. The right side of the purple line alone will also yield negligible contributions to the differentiation of the MH. (Right) $\Delta m_{\phi}^{2}$ for both electron antineutrino $\left(\Delta m_{\phi e e}^{2}\right)$ and muon disappearance $\left(\Delta m_{\phi \mu \mu}^{2}\right)$ are shown as a function of the unknown CP phase $\delta_{C P}$. Reproduced with permission from Ref. [6.9].

\subsection{Interpretation of Mass Hierarchy Sensitivity}

The determination of the neutrino $\mathrm{MH}$ is equivalent to finding the sign of $\Delta m_{32}^{2}=m_{3}^{2}-m_{2}^{2}$. Thus, the determination of $\mathrm{MH}$ represents the test of two discrete hypotheses (normal hierarchy vs. inverted hierarchy or NH vs. IH in short). In this context, there are two different but related questions: i) given the data from an experiment, how to quantify the finding regarding MH? and ii) when designing an experiment, how to evaluate its sensitivity to $\mathrm{MH}$ ? 

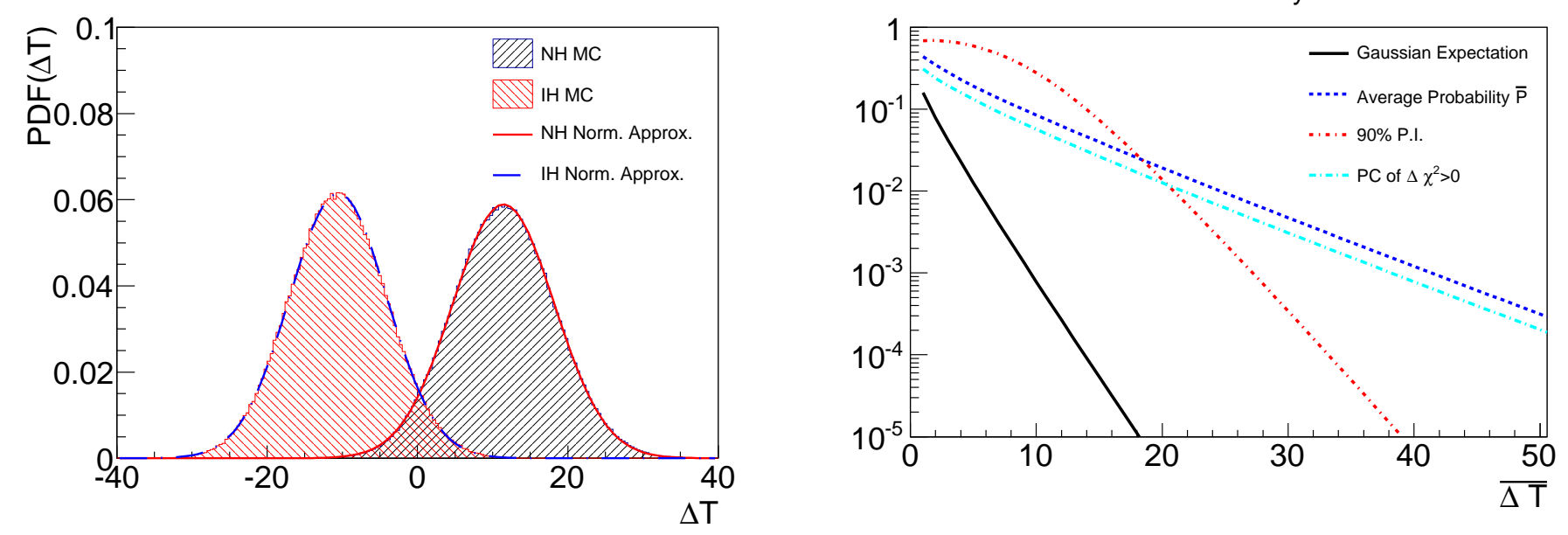

Figure 16: (Left panel) The probability distribution function of $\Delta T$ with $\overline{\Delta T} \approx 11$ is shown. The overlap is due to natural spread from statistical and systematic uncertainties. (Right panel) Several sensitivity metrics in the Bayesian statistics with respect to $\overline{\Delta T} \approx \Delta \chi_{\mathrm{MH}}^{2}$ are shown. See the text for details. Reproduced with the permission from Ref. [r6].

The usual approach to the first question is as follows. Given the result of a measurement, we compare the data (represented by " $\mathrm{x}$ ") with expectations from both the $\mathrm{NH}$ and $\mathrm{IH}$ hypotheses. In practice, one commonly defines a test statistic $T_{N H}:=-2 \log L_{N H}$, where $L_{N H}$ is the likelihood of NH for data "x". $T_{N H}$ is further minimized over all nuisance parameters, including unknown parameters and systematic uncertainties, to obtain $T_{N H}^{\min }$. Similarly, another test statistic $T_{I H}^{\min }$ is calculated from the likelihood of $\mathrm{IH}$ for data " $\mathrm{x}$ ". In order to evaluate whether the experimental data favors $\mathrm{NH}$ or $\mathrm{IH}$, a test statistics $\Delta T=T_{I H}^{\min }-T_{N H}^{\min }$ is defined. It is easy to see that a positive $\Delta T$ would favor the NH hypothesis, and a negative $\Delta T$ would favor the IH hypothesis. In addition, the absolute size of $\Delta T$ contains the information of how much data favor one hypothesis relative to the other one.

Regarding the second question, when designing an experiment, the community sometimes report the average expectation $\overline{\Delta T}$, which is calculated using the following procedure:

- Assuming that the true $\mathrm{MH}$ is $\mathrm{NH}$, a data set can be generated based on the best known experimental parameters. Such a data set does not include any statistical fluctuations and is usually called the typical data set or the Asimov data set [75].

- When this typical data set is compared with the NH hypothesis, we naturally have $T_{N H}^{\min }=0$. Therefore, $\overline{\Delta T_{M H=N H}}=T_{I H}^{\min }$. The subscript labels represent the assumption that the true $\mathrm{MH}$ is NH. Similarly, one can calculate $\overline{\Delta T_{M H=I H}}$ assuming that the true MH is IH. Here, $\overline{\Delta T}$ can be interpreted as either the most probable value of $\Delta T$ or the average value of $\Delta T$.

In reality, the data from any experiments always contain statistical fluctuations as well as variations due to systematics. Therefore, if the experiment would be repeated many times, a distribution of $\Delta T$ appears. Due to the nature of two discrete hypotheses of testing $\mathrm{MH}$, when the observed counts in the experiment are large enough, one can show that the distribution of $\Delta T$ approximately follows a Gaussian distribution with the mean and the standard deviation as $\overline{\Delta T}$, and $2 \sqrt{|\overline{\Delta T}|}$, respectively [76, 77, 78]. For example, the left panel of Fig. [6] shows the expected distribution of $\Delta T$ for experiments with $\left|\overline{\Delta T_{M H=N H}}\right| \approx\left|\overline{\Delta T_{M H=I H}}\right| \approx 11$. There will be about $4.8 \%$ of experiments which favor a wrong $\mathrm{MH}$

\footnotetext{
${ }^{2}$ The notation of $\overline{\Delta \chi^{2}}$ or sometimes $\Delta \chi^{2}$ is commonly used to represent $\overline{\Delta T}$.
} 


\begin{tabular}{|l|l|l|l|l|l|}
\hline$\Delta \tau \approx \Delta T$ & 1 & 4 & 9 & 16 & 25 \\
\hline $\mathrm{P}(\mathrm{IH} ; \mathrm{x})$ vs. $\mathrm{P}(\mathrm{NH} ; \mathrm{x})$ & $38 \%$ vs. & $12 \%$ vs. & $1.1 \%$ vs. & $0.034 \%$ vs. & $3.7 \times 10^{-6}$ vs. \\
& $62 \%$ & $88 \%$ & $98.9 \%$ & $99.966 \%$ & $100 \%$ \\
\hline $\mathrm{P}(\mathrm{IH} ; \mathrm{x}) / \mathrm{P}(\mathrm{NH} ; \mathrm{x})$ & 0.61 & 0.136 & 0.011 & $3.4 \times 10^{-4}$ & $3.7 \times 10^{-6}$ \\
\hline \hline
\end{tabular}

Table 2: Probability ratios are shown with respect to $\Delta \tau$ values.

preference. Therefore even if $\overline{\Delta T}$ is large, there remains a probability that a small $\Delta T$ is observed in an experiment. For example, in a situation where NH and IH are such that $|\overline{\Delta T}|=25$, the chance is about $1 \%$ that the experiment generates observed data favoring the wrong mass hierarchy. It should be noted that such a result naturally has a small $\Delta T$ value.

\subsubsection{Bayesian Approach to the Mass Hierarchy Determination}

As illustrated above given data from a measurement one can calculate $T_{N H}:-2 \log \left(L_{N H}\right)$ and $T_{I H}$ : $-2 \log \left(L_{I H}\right) . T_{N H}$ and $T_{I H}$ are further marginalized over all nuisance parameters including unknown parameters and systematic uncertainties of the experiment to obtain $T_{N H}^{\text {marginalized }}$ and $T_{I H}^{\text {marginalized }}$, respectively. ${ }^{\text {s }}$ Assuming that the prior information of $\mathrm{NH}$ vs. IH is $50 \%$ vs. $50 \%$, i.e. that both possibilities are equally expected, the probability ratio of the $\mathrm{IH}$ vs. $\mathrm{NH}$, which is $\mathrm{P}(\mathrm{IH} ; \mathrm{x})$ vs. $\mathrm{P}(\mathrm{NH} ; \mathrm{x})$, can be calculated as $e^{-\Delta \tau / 2}$ vs. 1 , with $\Delta \tau=T_{I H}^{\text {marginalized }}-T_{N H}^{\text {marginalized }}$ [[6]. Here, $e^{-\Delta \tau / 2}$ is commonly referred to as the Bayes factor. As illustrated in Ref. [[6] ], an approximation $\Delta \tau \approx \Delta T$ can be made in practice. More generally, $\Delta \tau$ can be explicitly calculated through MC simulations or other advanced integration techniques. Table $\nabla$ lists a few values of $\Delta \tau$ and their corresponding probability ratios. In the Bayesian approach, the final results are presented in terms of a probability ratio which is a natural and simple way to present results for the $\mathrm{MH}$ determination [76, 79].

Ref. [76] also provides a few sensitivity metrics based on the Bayesian approach that illustrate the capability of an experiment; these are useful in designing experiments. One of them is $\overline{P_{M H}^{N H}=N H}$ : the average probability to obtain the correct mass hierarchy. The second one is $P_{M H=N H}^{90 \%}$, which is based on probability intervals. The interpretation of $P_{M H=N H}^{90 \%}$ is the following: given that $\mathrm{NH}$ is the truth, $90 \%$ of the potential data would yield a probability of $\mathrm{NH}, \mathrm{P}(\mathrm{NH}$; data $\mathrm{x})$, higher than $P_{M H=N H}^{90 \%}$. Right panel of Fig. [6] shows the metrics $\overline{P_{M H=N H}^{N H}}$ (dotted blue line) and $P_{M H=N H}^{90 \%}$ (dotted red line) with respect to $\overline{\Delta T}$, assuming $\overline{\Delta T} \approx\left|\overline{\Delta T_{M H=N H} \mid} \approx\right| \overline{\Delta T_{M H=I H}} \mid$ and $\Delta \tau \approx \Delta T$.

\subsubsection{Frequentist Approach to the Mass Hierarchy Determination}

Ref. [7] (Particle Data Group) contains a very nice review regarding hypothesis tests. Given a null hypothesis $\mathrm{H}_{0}$ and the alternative hypothesis $\mathrm{H}_{1}$, type-I error rate $\alpha$ is defined as the probability of rejecting the null hypothesis $\mathrm{H}_{0}$, if $\mathrm{H}_{0}$ is true. On the other hand, type-II error rate $\beta$ is defined as the probability of accepting the null hypothesis $\mathrm{H}_{0}$, if $\mathrm{H}_{1}$ is true. Furthermore, according to NeymanPearson lemma, the likelihood ratio test is shown to be the most powerful test given that $\mathrm{H}_{0}$ and $\mathrm{H}_{1}$ are both simple hypotheses. ${ }^{⿴ 囗 十}$ This provides the basis to use $\Delta T$ defined above to perform the $\mathrm{MH}$ hypothesis test. Before data are obtained, we choose the type-I error rate $\alpha$, and this will decide the rule of the hypothesis test. The $\alpha$ value will determine the rejection region, and it will in turn determine the corresponding type-II error rate $\beta$. In our $\mathrm{MH}$ problem, one can choose the null hypothesis to be $\mathrm{NH}$,

\footnotetext{
${ }^{3}$ In the marginalization process, one integrates the likelihood function over the entire parameter space of the nuisance parameters.

${ }^{4} \mathrm{~A}$ simple hypothesis should not contain any undetermined parameters.
} 
and the alternative hypothesis would be IH. The result of this test will tell us whether the NH would be rejected or not, given the pre-defined rule. Similarly, one should also perform a second hypothesis test by choosing null hypothesis to be IH with the alternative hypothesis being NH. The result of the second test will tell us whether the IH would be rejected or not. Unlike the Bayesian approach, the Frequentist approach does not directly address the question how much one MH hypothesis is favored over the other MH hypothesis given the experimental data.

Furthermore, given experimental data and test statistics, one can obtain the p-value. The p-value represents the probability of obtaining potential data that are less compatible with the null hypothesis compared to the current experimental data. We emphasize that one should not confuse the p-value in Frequentist approach with the probability ratios in the Bayesian approach. For the MH determination, a nice feature exists for the p-value of the null hypothesis $\mathrm{H}_{0}$ being the disfavored $\mathrm{MH}$ hypothesis. In order to illustrate this point, we use the simple model shown in the left panel of Fig. ए6]. Given $\Delta T$ from data and $\overline{\Delta T}$ from the design of the experiment, the number of standard deviations which corresponds to the 1-sided p-value of the null hypothesis $\mathrm{H}_{0}$ being the disfavored $\mathrm{MH}$ hypothesis can be written as:

$$
(\text { number of } \sigma)=\frac{(|\Delta T|+\sqrt{|\overline{\Delta T}|})}{2 \sqrt{|\overline{\Delta T}|}} \geq \sqrt{|\Delta T|}
$$

The equal sign is reached when $|\Delta T|=|\overline{\Delta T}|$. In other words, given the observed data $\Delta T$, there is a maximum $\mathrm{p}$-value of the null hypothesis $\mathrm{H}_{0}$ being the disfavored $\mathrm{MH}$ hypothesis, which corresponds to $\sqrt{|\Delta T|}$ in terms of the number of standard deviations. ${ }^{\text {a }}$ We should further point out that this maximum p-value does not depend on $\overline{\Delta T}$.

The sensitivity of an experimental design can be represented using the type-I error rate $\alpha$ at certain type-II error rate $\beta$. Given a design of an experiment, the type-I error rate $\alpha$ and type-II error rate $\beta$ are directly connected. In other word, $\alpha$ can be expressed as a function of $\beta$. A smaller type-I error rate $\alpha$ would lead to a larger type-II error rate $\beta$ and vice versa. The community commonly uses $\sqrt{\overline{\Delta \chi^{2}}}$ to represent the MH sensitivity of an experimental design. A recent discussion can be found in Ref. [80]]. In a simplified model shown in the left panel of Fig. [6], the choice of $\sqrt{\overline{\Delta \chi^{2}}}$ corresponds to choosing the value of type-I error rate $\alpha$ as the sensitivity metric, while type-II error rate $\beta$ is fixed at $0.5(\beta=0.5)$.

Through investigating the statistical problem regarding Spin discrimination of the Higgs boson, whose nature is very similar to the MH discrimination, authors of Ref. [ㅍ] suggested to consider the value of $\alpha(\beta=\alpha)$, which is the value of $\alpha$ when $\alpha=\beta$, as the sensitivity metric. This can be compared to the conventional sensitivity metric $\alpha(\beta=0.5)$, which is the value of $\alpha$ at $\beta=0.5$. In the simplified model as shown in the left panel of Fig. [6], the latter sensitivity coincides with the sensitivity metric $F_{M H=N H}$ in Ref. [[6] , which is the probability of obtaining a $\Delta T$ favoring the correct MH. Right panel of Fig. [6] shows $F_{M H=N H}$ with respect to $\overline{\Delta T}$ (cyan dash-dotted line).

In general, the high energy physics community is more familiar with the Frequentist approach than the Bayesian approach. In particular, there exist traditional criteria regarding the evidence and discovery of new physics: the simple $3 \sigma$ (evidence) and $5 \sigma$ (discovery) rule. If the 1-sided p-value of data when compared to the null hypothesis is smaller than $0.13 \%$, one can claim that the evidence of new physics is observed. Similarly, if the 1 -sided p-value is smaller than $3 \times 10^{-7}$, one can claim the discovery of new physics. For example, in the case of the non-zero $\theta_{13}$ discovery [37], the null hypothesis is $\theta_{13}=0$. In the case of the discovery of Higgs boson [ $[\mathbf{8 2}, \mathbf{8 3}]$, the null hypothesis is the non-existence of Higgs boson.

Recently, there have been some inspiring discussions [84, 85] on whether the $5 \sigma$ rule should be universally applied as the criteria for discoveries. The $5 \sigma$ rule was invented to take into account i) look-

\footnotetext{
${ }^{5}$ When transformed into number of standard deviations, a smaller p-value would give a bigger number.
} 
elsewhere effect and ii) hidden unknown systematic uncertainties. For the $\mathrm{MH}$ determination, there is clearly no look-elsewhere effect. In this case, the discovery criteria should be adjusted accordingly. We further note that in practice it is strongly preferable that the designed experiment is not dominated by systematic uncertainties.

\section{$3 \quad$ Experiments}

In this section, we briefly describe the existing and planned experiments that have sensitivities to the neutrino mass hierarchy. In the first part, the current status is discussed. While the analysis of the atmospheric neutrino data by the Super-Kamiokande experiment slightly favors the normal hierarchy, the comparison of the experimentally determined $\Delta m_{32}^{2}$ mass splitting using the muon and electron neutrino disappearance favors the inverted hierarchy. These two hints, that so far lack statistical significance, are obviously incompatible with each other. The running NO$\nu \mathrm{A}$ experiment will be able to shed some light on this issue, but needs to collect more data. Also, there is a significant degeneracy between $\mathrm{MH}$ and the value of the $\mathrm{CP}$ phase $\delta_{C P}$ due to the baseline of $\mathrm{NO} \nu \mathrm{A}$.

In the second part of the section we describe the proposed and planned experiments that promise to resolve MH. They are based on the study of the accelerator neutrinos (DUNE/LBNF), atmospheric neutrinos (PINGU, ORCA, INO, and Hyper-Kamiokande), and reactor neutrinos (JUNO, RENO-50). These next-generation experiments are in various stages of planning and funding.
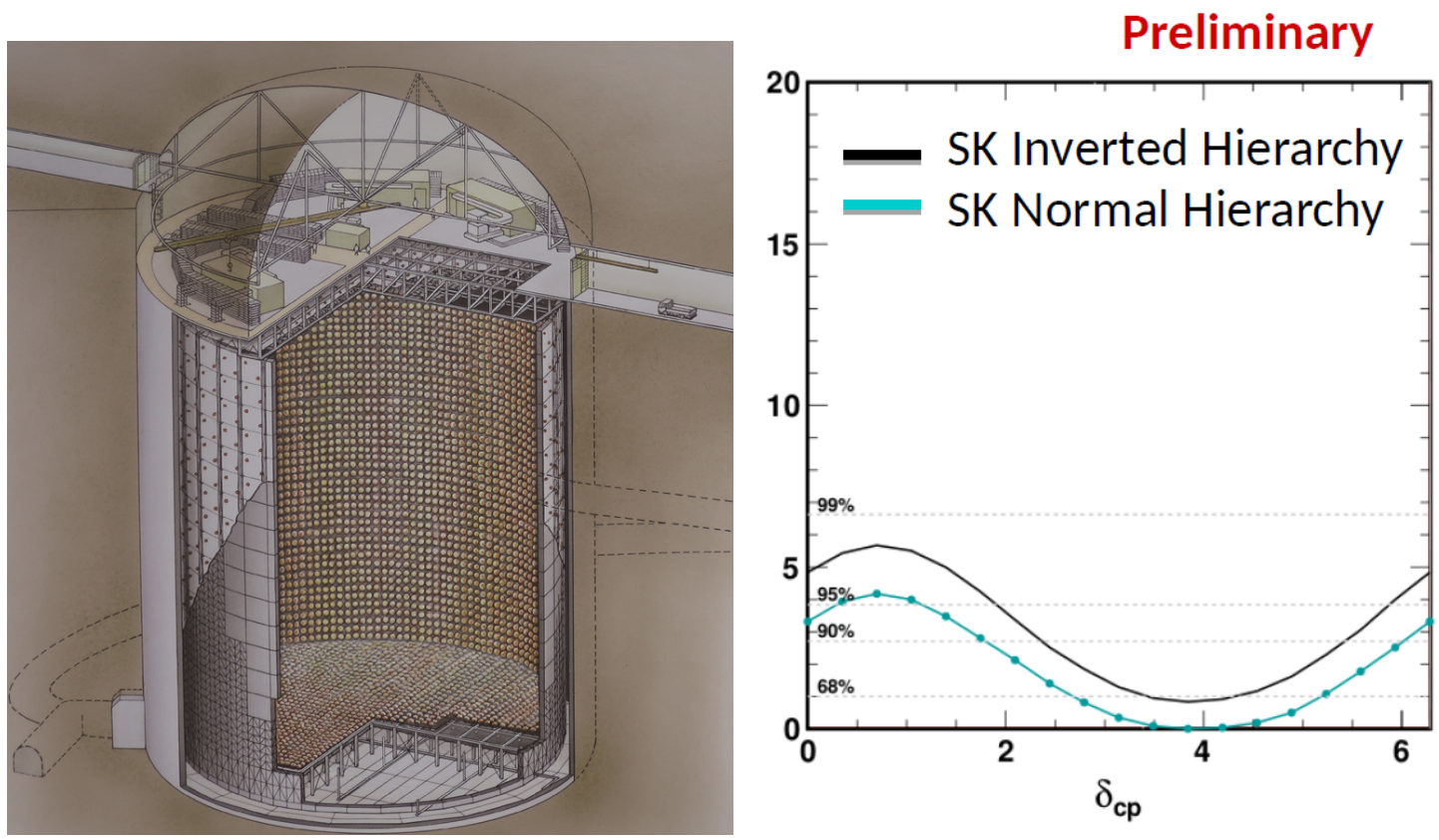

Figure 17: (Left) A schematic view of the Super-Kamiokande detector. Reproduced with the permission of Kamioka Observatory, ICRR (Institute for Cosmic Ray Research), the university of Tokyo. (Right) Preliminary result of Super-Kamiokande mass hierarchy measurement in terms of the negative-two-loglikelihood ratio ( or $\Delta \chi^{2}=\chi^{2}-\chi_{\text {min }}^{2}$ ) are plotted as a function of the unknown CP phase $\delta_{C P}$. The normal mass hierarchy is slightly favored by $\Delta \chi^{2} \sim 0.9$, corresponding to about $60 \%$ vs. $40 \%$ probability with the Bayesian interpretation. This plot is reproduced with the permission from Ref. [ 86$]$ ]. 


\subsection{Current Status}

\subsubsection{Atmospheric neutrino results from Super-Kamiokande}

The analysis of the atmospheric neutrino data from the Super-Kamiokande experiment currently provides a hint of the mass hierarchy. The Super-Kamiokande detector (left of Fig. 미) is a cylindrical 50-kton (22.5-kton fiducial volume) water Cherenkov detector located $1000 \mathrm{~m}$ underground in western Japan. The signal readout uses an array of regularly spaced large photo-multiplier tubes sensitive to single photo-electrons [87]. From the pattern of the detected Cherenkov light, one can reconstruct the energy, the direction, as well as the particle type of the charged leptons produced by the charged-current neutrino-nuclear interaction. Using the principle illustrated in Sec. 2.3, Super-Kamiokande's atmospheric neutrino data are sensitive to MH. Preliminary result was presented at Neutrino 2014 [ 86, , 88] and shown in the right panel of Fig. ㅁ․ The normal mass hierarchy is slightly favored by $\Delta \chi^{2} \sim 0.9$. When interpreted in the Bayesian framework, this preliminary result corresponds to $60 \%$ (normal) vs. $40 \%$ (inverted) probability in favor of the normal hierarchy.

\subsubsection{Global Analysis of the $\left|\Delta m_{32}^{2}\right|$ Mass-squared Splitting}
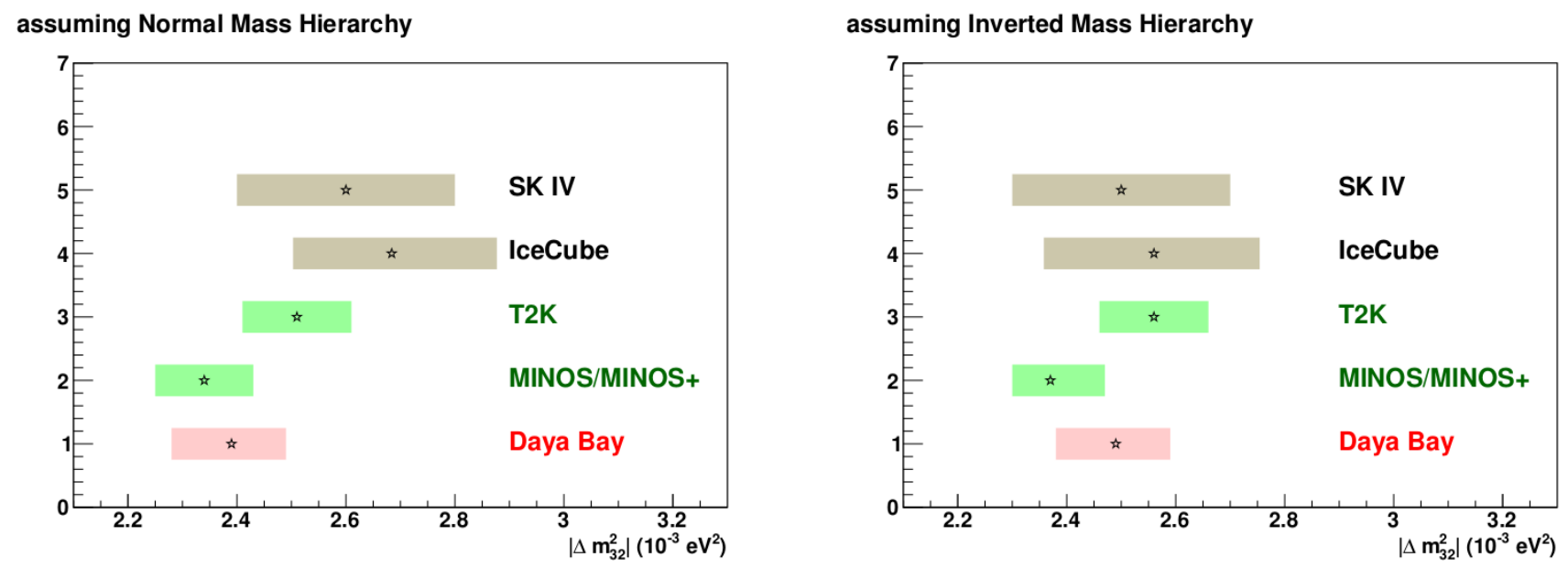

Figure 18: $\left|\Delta m_{32}^{2}\right|$ reported at the Neutrino 2014 conference are summarized assuming normal (left) and inverted (right) mass hierarchy scenarios. While Daya Bay measures the $\bar{\nu}_{e}$ disappearance using the reactors, the rest of experiments measure the $\nu_{\mu}$ and/or $\bar{\nu}_{\mu}$ disappearance using accelerator neutrinos (T2K and MINOS/MINOS+) and atmospheric neutrinos (Super-Kamiokande and IceCube).

As illustrated in Sec. 2.4, MH information can be also extracted by comparing the measurement of the effective atmospheric mass-squared splitting, i.e. the value of $\Delta m_{32}^{2}$, between the electron and muon neutrino disappearance channels. For the normal (inverted) mass hierarchy, the effective masssquared splitting in electron neutrino disappearance channel is larger (smaller) than that in the muon neutrino disappearance channel. In other word, the $\Delta m_{32}^{2}$ value extracted from these two channels would be more consistent with each other when testing the true $\mathrm{MH}$ hypothesis than the incorrect $\mathrm{MH}$ hypothesis. Figure 18 shows the extracted $\left|\Delta m_{32}^{2}\right|$ from Super Kamiokande [86] in Japan, IceCube [89] at the South Pole, T2K [90] in Japan, MINOS/MINOS+ [91] in the U.S., and Daya Bay [30, 92] in China at the Neutrino 2014 conference under both normal and inverted MH scenarios. While Daya Bay measures the $\bar{\nu}_{e}$ disappearance from reactors, the rest of experiments measure the $\nu_{\mu}$ and/or $\bar{\nu}_{\mu}$ disappearance. Figure $\mathbb{\nabla}$ shows that IH are slightly favored compared to NH. Assuming all results are uncorrelated, the fit of $\mathrm{NH}$ results yields a $\chi^{2} / N D F$ value of 4.4/4. On the other hand, the fit of IH 
results yield a $\chi^{2} / N D F$ value of $1.85 / 4$. Therefore, the IH is favored by $\Delta \chi^{2} \sim 2.55$, which corresponds to a probability ratio of $78 \%$ vs. $22 \%$ in the Bayesian framework.

\subsection{3 $\quad \mathrm{NO} \nu \mathrm{A}$}

The NO $\nu \mathrm{A}$ (NuMI Off-axis $\nu_{e}$ Appearance) experiment [9,3] is an accelerator neutrino experiment in the United States. It is currently taking data. NO $\nu$ A measures the $\nu_{e}\left(\bar{\nu}_{e}\right)$ appearance as well as the $\nu_{\mu}\left(\bar{\nu}_{\mu}\right)$ disappearance in a $\nu_{\mu}\left(\bar{\nu}_{\mu}\right)$ beam. The neutrino beam is generated by the $120 \mathrm{GeV}$ proton beam from the Fermilab's Main Injector. The system consists of two detectors, with the detector near the production point characterizing the neutrino beam and the far detector at the baseline of $810 \mathrm{~km}$ studying neutrino oscillations. Left panel of Fig. [प shows the overall configuration of the NO $\nu \mathrm{A}$ experiment. The far detector is located at $14 \mathrm{mrad}$ off-axis of the incident proton beam to obtain a narrow band neutrino beam around $2 \mathrm{GeV}$ energy. The $810 \mathrm{~km}$ baseline corresponds to the maximal oscillation at $2 \mathrm{GeV}$. In addition, the long baseline offers the experiment a chance to determine the mass hierarchy through the matter effect as discussed in Sec. [2.2].
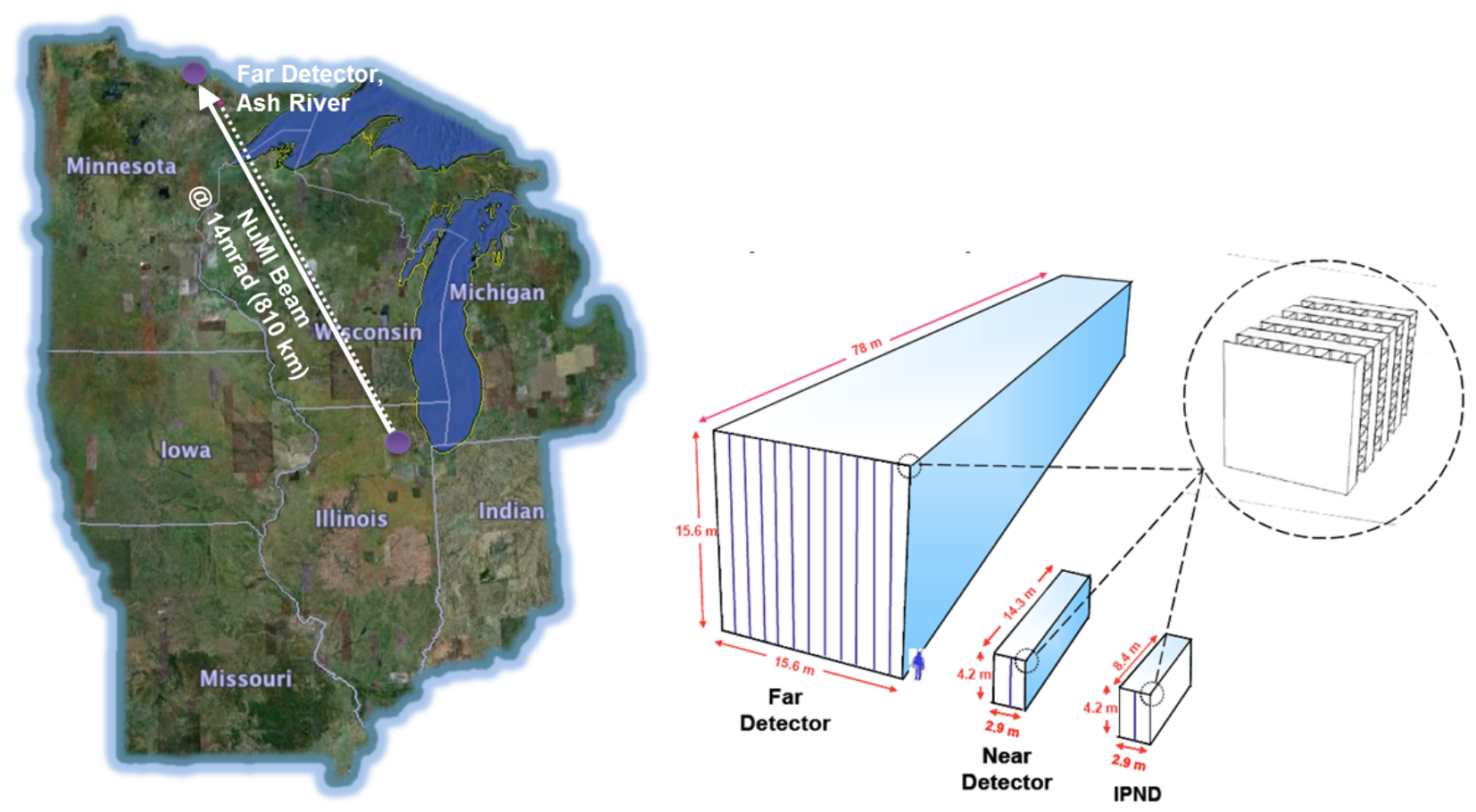

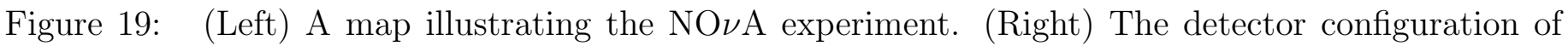
$\mathrm{NO} \nu \mathrm{A}$ from Ref. [94]. Reproduced with the permission from Ref. [95].

Right panel of Fig. [1. shows the detector configuration of $\mathrm{NO} \nu \mathrm{A}$ [94]. The near (0.3 kt) and far (14 kt) detectors are structurally and functionally identical, despite the difference in their absolute sizes. Such a configuration allows to minimize the systematic uncertainties in the neutrino beam, the neutrino interaction cross section, and the detecting efficiency. The near detector consists of 18 thousand PVC cells filled with liquid scintillator and arranged in alternating X/Y layers with an overall dimension of $14.3 \times 4.2 \times 4.2 \mathrm{~m}^{3}$. The light is transported through a wavelength shifting fiber and then read out with avalanche photodiode. The far detector is approximately 60 times larger with the overall dimension of $60 \times 15.6 \times 15.6 \mathrm{~m}^{3}$. Since it is located on the surface, the far detector is exposed to a large flux of cosmic muons. Nevertheless, a factor of $4 \times 10^{7}$ suppression has been achieved to reject the cosmic ray background, taking into account the timing and the direction of the $\nu_{\mu}$ and $\nu_{e}$ beam. Figure 201 shows the expected $\mathrm{MH}$ sensitivity from $\mathrm{NO} \nu \mathrm{A}$ after collecting a total of $36 \times 10^{20}$ protons on target (POT), 
which corresponds to about six years (three years neutrino and three years antineutrino running) of data taking at $750 \mathrm{~kW}$ beam power. The mass hierarchy resolution has a large dependence on the value of the unknown $\mathrm{CP}$ phase $\delta_{C P}$. This is so because both the matter effect and the CP phase $\delta_{C P}$ can induce differences between neutrino and antineutrino appearance oscillations, which leads to a degeneracy between the $\mathrm{MH}$ determination and the determination of $\mathrm{CP}$ phase $\delta_{C P}$ for certain values of $\delta_{C P}$. At the most favorable $\mathrm{CP}$ values, the sensitivity of $\mathrm{MH}$ determination can reach about $3 \sigma$. At this sensitivity, there is a $50 \%$ chance that $\mathrm{NO} \nu \mathrm{A}$ will be able to reject the unfavored $\mathrm{MH}$ hypothesis by $3 \sigma$. Meanwhile, $6.7 \%$ of measurements will yield a result favoring the wrong $\mathrm{MH}$ hypothesis.

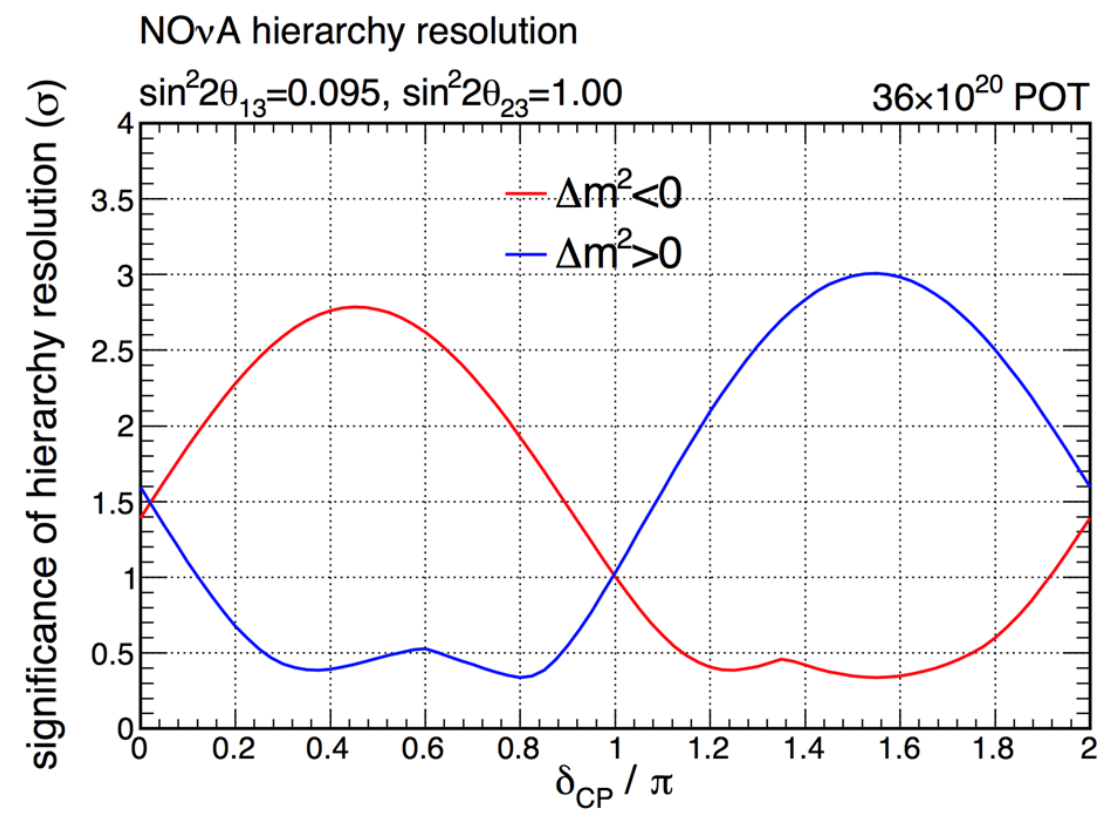

Figure 20: Mass hierarchy sensitivity from $\mathrm{NO} \nu \mathrm{A}$ (taken from Ref. [95]) is shown. The "significance of hierarchy resolution" is defined as $\sqrt{\Delta \chi^{2}}:=\sqrt{\bar{T}}$ as introduced in Sec. ए.5.

\subsection{Proposed and Planned Experiments}

\subsubsection{DUNE/LBNF}

In this subsection the long baseline experiments that aim at the determination of the neutrino mass hierarchy, among other things, are described. There were two proposals, the Long-Baseline Neutrino Experiment (LBNE) [96] in the US and the Large Apparatus for Grand Unification and Neutrino Astrophysics and Long Baseline Neutrino Oscillations (Laguna-LBNO or LBNO) [97] in Europe, designed to separate the signal of the two hierarchies with matter effect. While the fundamental goal of the two proposals is similar, they differ in important details, in particular in the length of the baseline. Due to the limited available resource and expected costs of these projects, LBNE and LBNO collaborations combined efforts and merged into a new experiment located in the US: the Deep Underground Neutrino Experiment at the Long-Baseline Neutrino Facility (DUNE/LBNF). We concentrate on the DUNE experiment and will describe it in details. There is no firm schedule for DUNE at the present time. Tentatively, the construction of DUNE/LBNF might begin in 2017 and the neutrino beam might be ready by $\sim 2023$.

DUNE is an accelerator neutrino experiment under development in the United States. It consists of a horn-produced broad band beam with $60-120 \mathrm{GeV}$ protons at $\sim 1 \mathrm{MW}$ from Fermilab, $>35$ kton fiducial volume Liquid Argon Time Projection Chambers (LArTPC) 4850-feet underground at Sanford Laboratory in Lead, South Dakota, as the far detector, and high-resolution near detector(s) at Fermilab. 
Figure 2$]$ shows the overall configuration of DUNE. In order to optimize the physics sensitivity to the CP phase $\delta_{C P}$, the baseline of DUNE is chosen to be $1300 \mathrm{~km}$ [98], which is longer than any existing accelerator neutrino experiments. The large underground LAr detector also represents an excellent opportunity for the underground science including proton decay searches, detection of atmospheric neutrinos and supernova neutrino burst. In particular, the detection of atmospheric neutrinos will provide additional information regarding $\mathrm{MH}$.

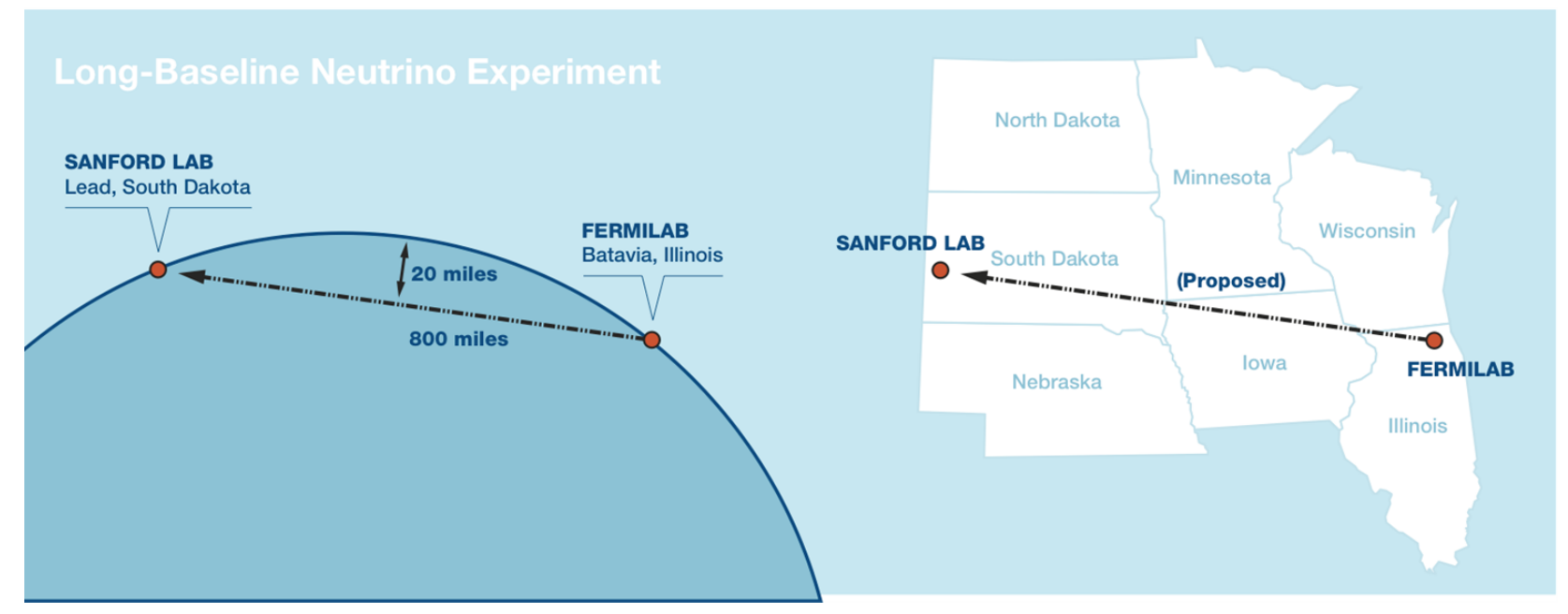

Figure 21: Illustration of the DUNE/LBNF (formerly known as the LBNE) experiment [99].
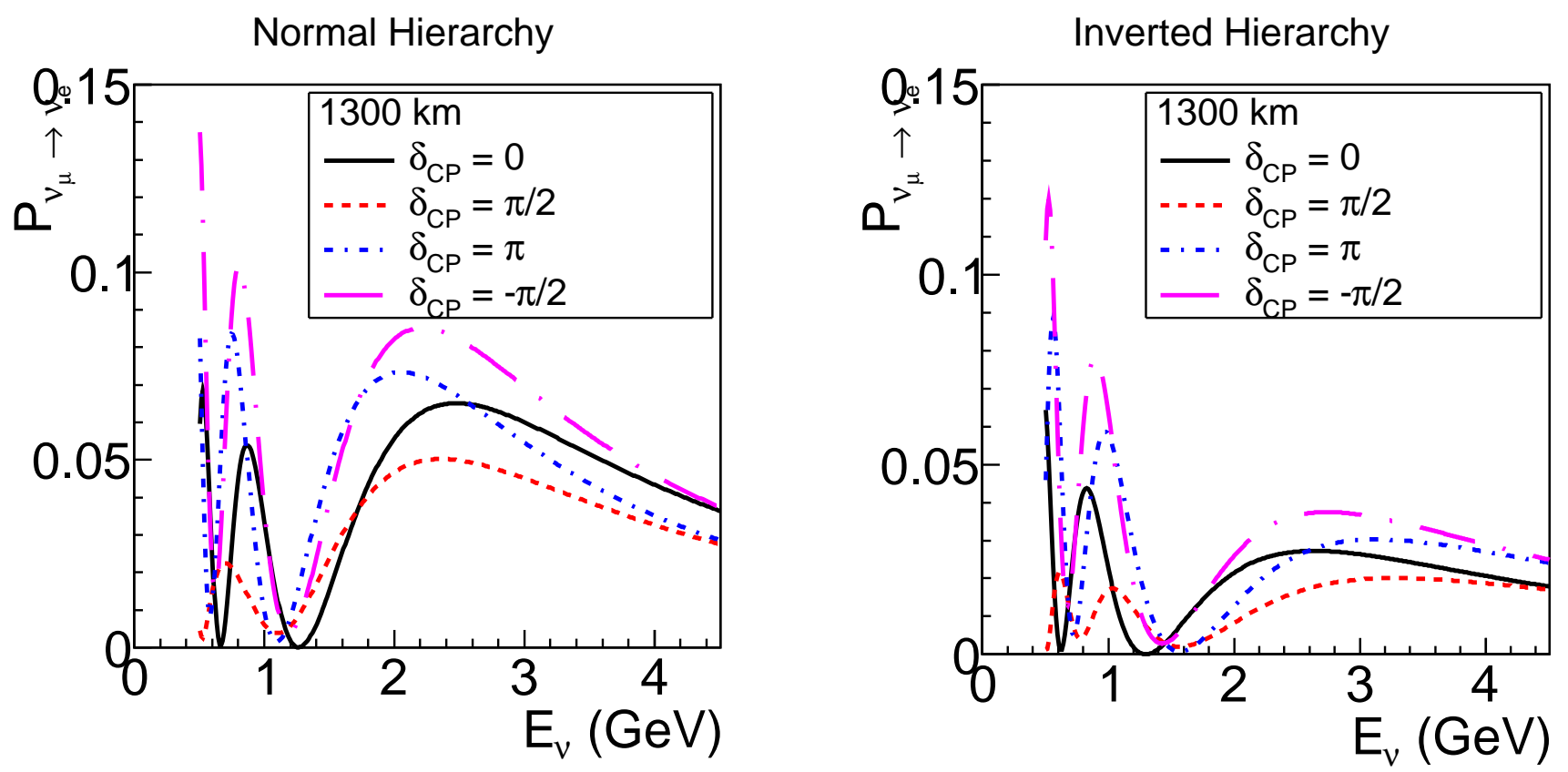

Figure 22: The $\nu_{\mu} \rightarrow \nu_{e}$ oscillation probabilities are shown for the normal (left) and the inverted (right) mass hierarchies. Different curves represent different values of currently unknown CP phase $\delta_{C P}$. The first and second oscillation maxima locate at about 2.5 and $0.8 \mathrm{GeV}$, respectively. The matter density $\rho$ and the electron fraction $Y_{e}$ are assumed to be $2.7 \mathrm{~g} / \mathrm{cm}^{3}$ and 0.5 , respectively.

DUNE is designed to precisely measure parameters that govern $\nu_{\mu} \rightarrow \nu_{e}, \bar{\nu}_{\mu} \rightarrow \bar{\nu}_{e}, \nu_{\mu} \rightarrow \nu_{\mu}$, and $\bar{\nu}_{\mu} \rightarrow \bar{\nu}_{\mu}$ oscillations, including the second mixing angle $\theta_{23}$, the third mixing angle $\theta_{13}$, the CP phase 
$\delta_{C P}$, and MH. Figure [2] shows the $\nu_{\mu} \rightarrow \nu_{e}$ oscillation probabilities for different values of $\delta_{C P}$ for both normal and inverted mass hierarchies. The principle of the MH determination in DUNE relies on the matter effect, introduced in Sec. 22. For $\mathrm{NH}$, the $\nu_{e}\left(\bar{\nu}_{e}\right)$ appearance probability at first oscillation maximum $(\sim 2.5 \mathrm{GeV})$ is enhanced (suppressed), while for $\mathrm{IH}$, the opposite is true, i.e., the $\nu_{e}\left(\bar{\nu}_{e}\right)$ appearance probability at first oscillation maximum is suppressed (enhanced). At $1300 \mathrm{~km}$, the matter effect in DUNE is large enough so that there is no fundamental degeneracy between the matter effect and the $\delta_{C P}$-induced $\mathrm{CP}$ effect. In other word, given enough statistics, these two effects can be totally disentangled. Furthermore, the precision measurements of the oscillation probability will also enable a measurement of the currently unknown $\mathrm{CP}$ phase $\delta_{C P}$ that may hold the key to explain the large matter over anti-matter asymmetry in our universe [u(0)].
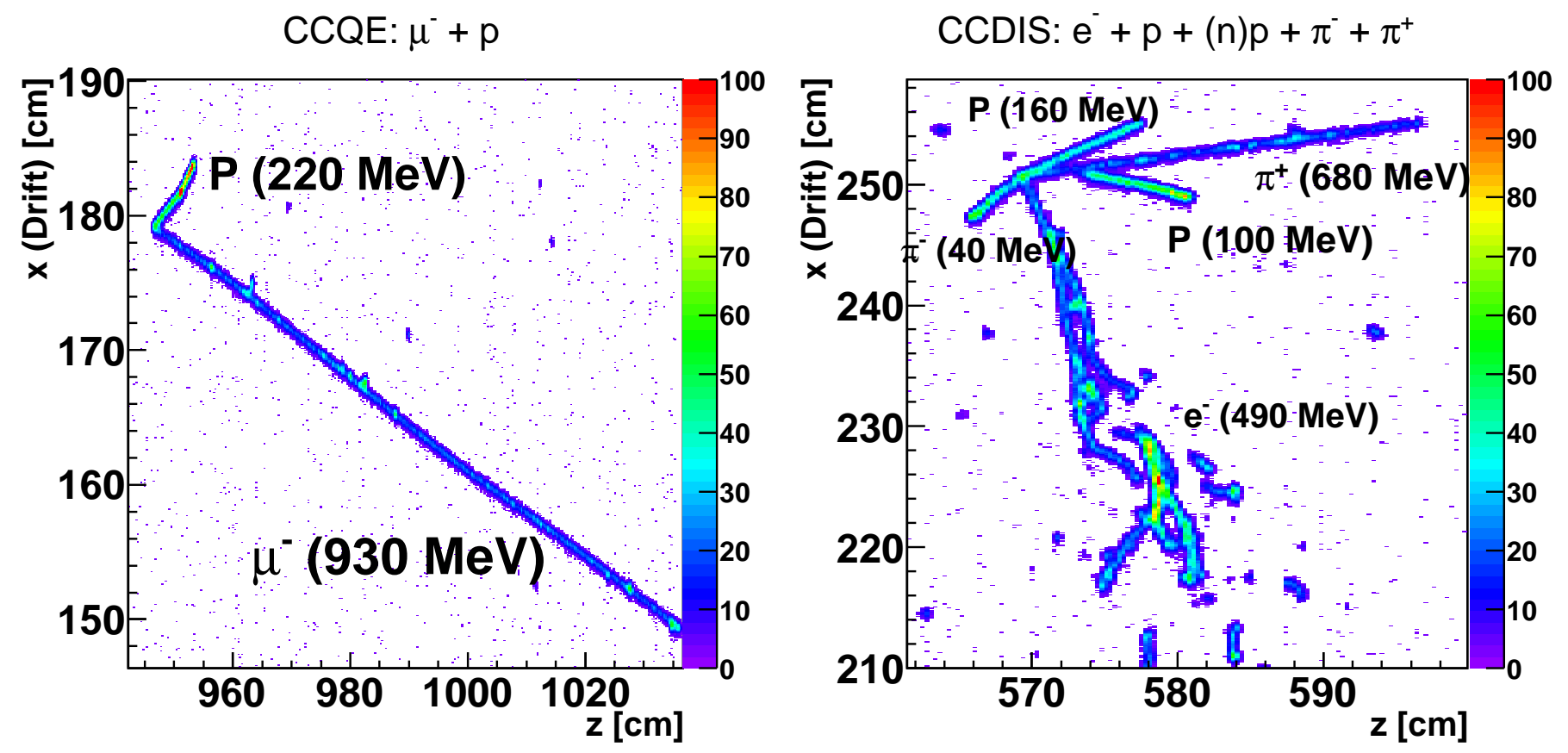

Figure 23: Event display of two neutrino events from Monte-Carlo. (Left) Charged-Current Quasi Elastic (CCQE) interaction with final states containing a muon and a proton. (Right) Charged-Current Deep-Inelastic-Scattering (CCDIS) with final states containing an electron shower, two protons (one being generated by neutron final state interaction), one positive pion, and one negative pion.

The detector technology in DUNE is chosen to be the liquid argon time projection chamber (LArTPC) [III]]. This technology was firstly proposed by C. Rubbia for the neutrino physics [102] in 1977. As the most abundant noble gas in the atmosphere $(\sim 1.3 \%$ by weight), argon can be extracted economically, and is an ideal material for the TPC. Electrons have very high mobility in LAr [103]. At $500 \mathrm{~V} / \mathrm{cm}$ electric field and $87 \mathrm{~K}$ temperature, the electron drift velocity is about $1.6 \mathrm{~km} / \mathrm{s}$ [104, 105, 106, 107], which is equivalent to about $0.64 \mathrm{~ms}$ for electrons to travel $1 \mathrm{~m}$. Electrons have low diffusions in LAr [108]; the longitudinal and transverse diffusion coefficients are about 5 and $13 \mathrm{~cm}^{2} / \mathrm{s}$ [ए0., Ш0, ШШ, Ш2], respectively at $500 \mathrm{~V} / \mathrm{cm}$ electric field. For a $1.6 \mathrm{~ms}$ drift time, these numbers correspond to a position resolution (one standard deviation) of about 1.3 and $2.0 \mathrm{~mm}$ for longitudinal and transverse diffusions, respectively. LAr also has relatively high density $(\sim 1.4 \mathrm{~g} / \mathrm{mL}$ at $87 \mathrm{~K})$, advantageous for neutrino experiments.

LAr is also a good scintillation material. The scintillation light (emission peaks at 128 nm [Ш3]) can provide the absolute event time to determine the drift distance along the electric field direction. The coordinate information perpendicular to the electron drift direction can be determined with a high resolution detector (e.g. wire planes). The spatial resolution could reach a few mm. Finally, the 
ionization energy required to produce an electron-ion pair is about $23.6 \mathrm{eV}$ [103, 114]. Some of the pairs will recombine in LAr, and the remaining electrons will be collected at wire planes. The charge collected per wire will be closely related to the energy deposition per unit distance (dE/dx), which can be used for the particle identification (PID). In addition, the event topology can be used for PID as well. LArTPCs are excellent detectors of neutrino interactions. Fig. $2: 3$ shows event displays for two neutrino events from Monte Carlo as an example. The high-resolution nature of LArTPC is self-evident.
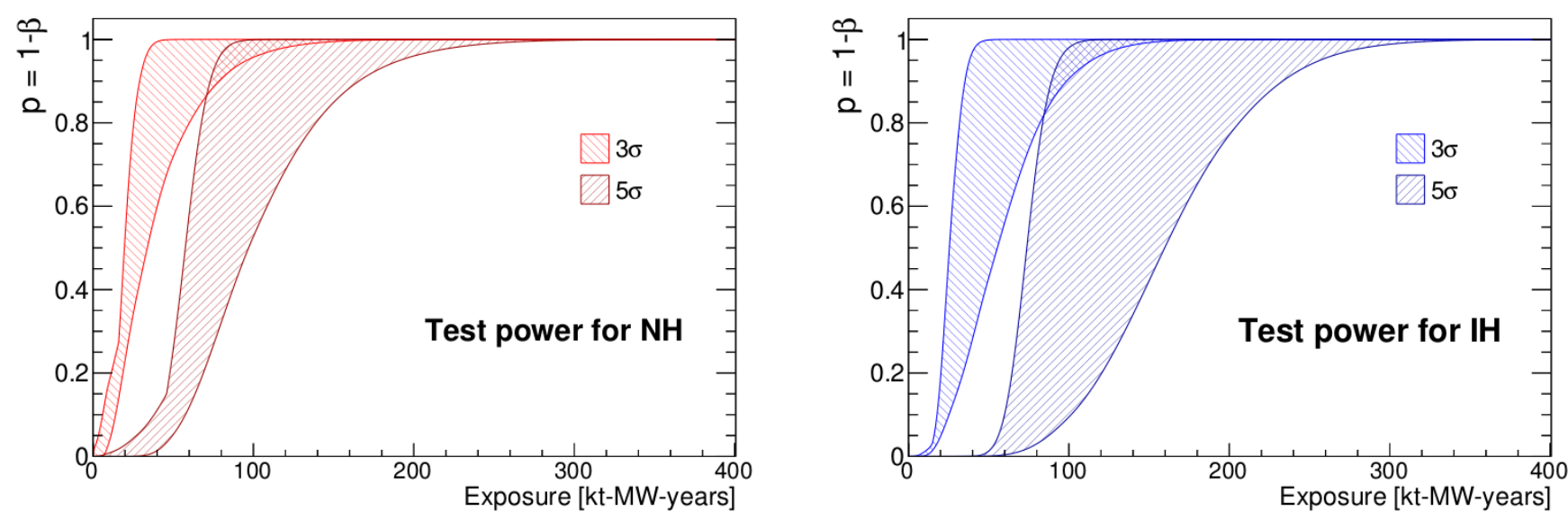

Figure 24: The test power $(p=1-\beta$ as introduced in Sec. 2.5$)$, which represents the probability of accepting the correct $(\mathrm{NH})$ hypothesis while excluding the incorrect $(\mathrm{IH})$ hypothesis at the 3 and $5 \sigma$ level, shown as a function of exposure in kt-MW-years [115]. The width of the bands represent the range of CP values. Sensitivities are for true NH (left) and true IH (right).
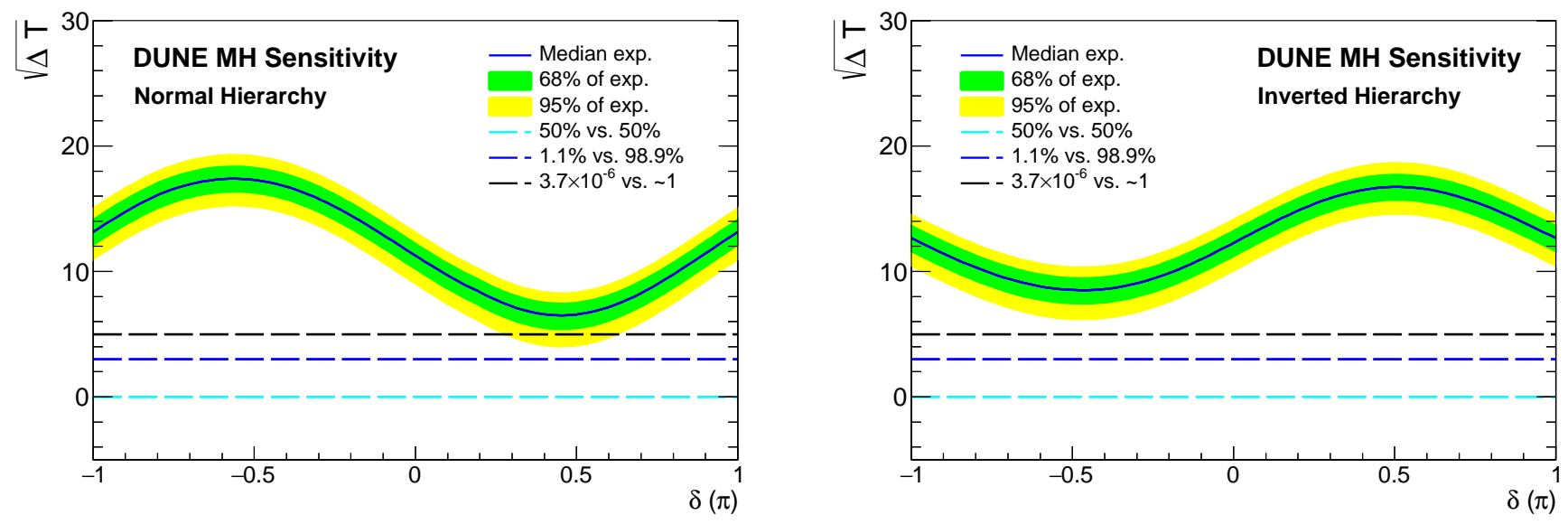

Figure 25: The sensitivity for a typical experiment (blue line) is compared to the bands within which $68 \%$ (green) and 95\% (yellow) of experiments are expected to fall [115]. The dashed lines represent the value of the $\sqrt{\Delta T}$ metric for which the probability of determining the correct $\mathrm{MH}$ is $50 \%$ (cyan), $98.9 \%$ (blue), or $3.7 \times 10^{-6}$ (black) in the Bayesian framework. The normal and inverted hierarchy are assumed to be true in the left and right plots, respectively.

In order to suppress the uncertainties in neutrino interaction cross sections and the neutrino flux, a fine-grained neutrino detector will be installed at FNAL near the beam source. The near detector will measure the absolute neutrino flux via i) neutral-current $\nu_{\mu}+e^{-} \rightarrow \nu_{\mu}+e^{-}$elastic scattering, ii) inverse-muon decay $\nu_{\mu}+e^{-} \rightarrow \mu^{-}+\nu_{e}$ for the high energy neutrino component, and iii) charged-current 
quasi-elastic scattering $\nu_{\mu}+n \rightarrow \mu^{-}+p$ and $\bar{\nu}_{\mu}+p \rightarrow \mu^{+}+n$. In addition, it will also measure the spectra of all four neutrino species, $\nu_{\mu}, \bar{\nu}_{\mu}, \nu_{e}$, and $\bar{\nu}_{e}$ through the low- $\nu_{0}$ method [116, 117] to enable an accurate prediction for each species of the far/near flux ratio as a function of energy. The fine grained detector will be able to precisely measure the four-momenta of secondary hadrons, such as the charged and neutral mesons produced, and to constrain neutrino interaction cross sections, which are crucial inputs to the oscillation analysis. Furthermore, the precise measurement of these hadrons produced in the neutral- and charged-current interactions will significantly improve the background estimation in the oscillation signal.

Fig. 24 shows the expected sensitivity of the $\mathrm{MH}$ determination as a function of the accumulated exposure in units of kt-MW-years. As introduced in Sec. [2.5.2, the test power $1-\beta$ is plotted at values of type-I error $\alpha$ corresponding to 3 and $5 \sigma$ significance. Fig. 25.5 show the sensitivity in terms of test statistic $\Delta T$ as introduced in Sec. 2.5.J. At $300 \mathrm{kt}-\mathrm{MW}$-years exposure, most of experiments will yield a $\mathrm{MH}$ determination better than $3.7 \times 10^{-6}$ vs. 1 in the Bayesian framework.

LBNO, before merging into DUNE/LBNF with LBNE, was a proposed experiment in Europe. The initial neutrino beam is expected to be produced from CERN with a $400 \mathrm{GeV}$ proton beam with a power of $750 \mathrm{~kW}$. The primary choice of the far detector location is the Pyhäsalmi mine in Finland. Various other sites are also under consideration. A multi-tens of kilotons double-phase liquid argon time projection chamber is expected to reside deep underground. The baseline from CERN to Pyhäsalmi is $2300 \mathrm{~km}$, much longer than that of LBNE $(1300 \mathrm{~km})$. The $\nu_{\mu} \rightarrow \nu_{e}$ oscillation patterns are shown in Fig. 26]. Comparing to those of $1300 \mathrm{~km}$ (Fig. [2]), the matter effect (leading to the MH determination) at $2300 \mathrm{~km}$ is better due to longer baseline and corresponding higher neutrino energies. Therefore, the MH sensitivity is expected to be much larger than that of $1300 \mathrm{~km}$. Given a similar overall exposure in kt-MW-years, it has a factor of $\sim 4$ increase in the test statistics $\overline{\Delta T}$.
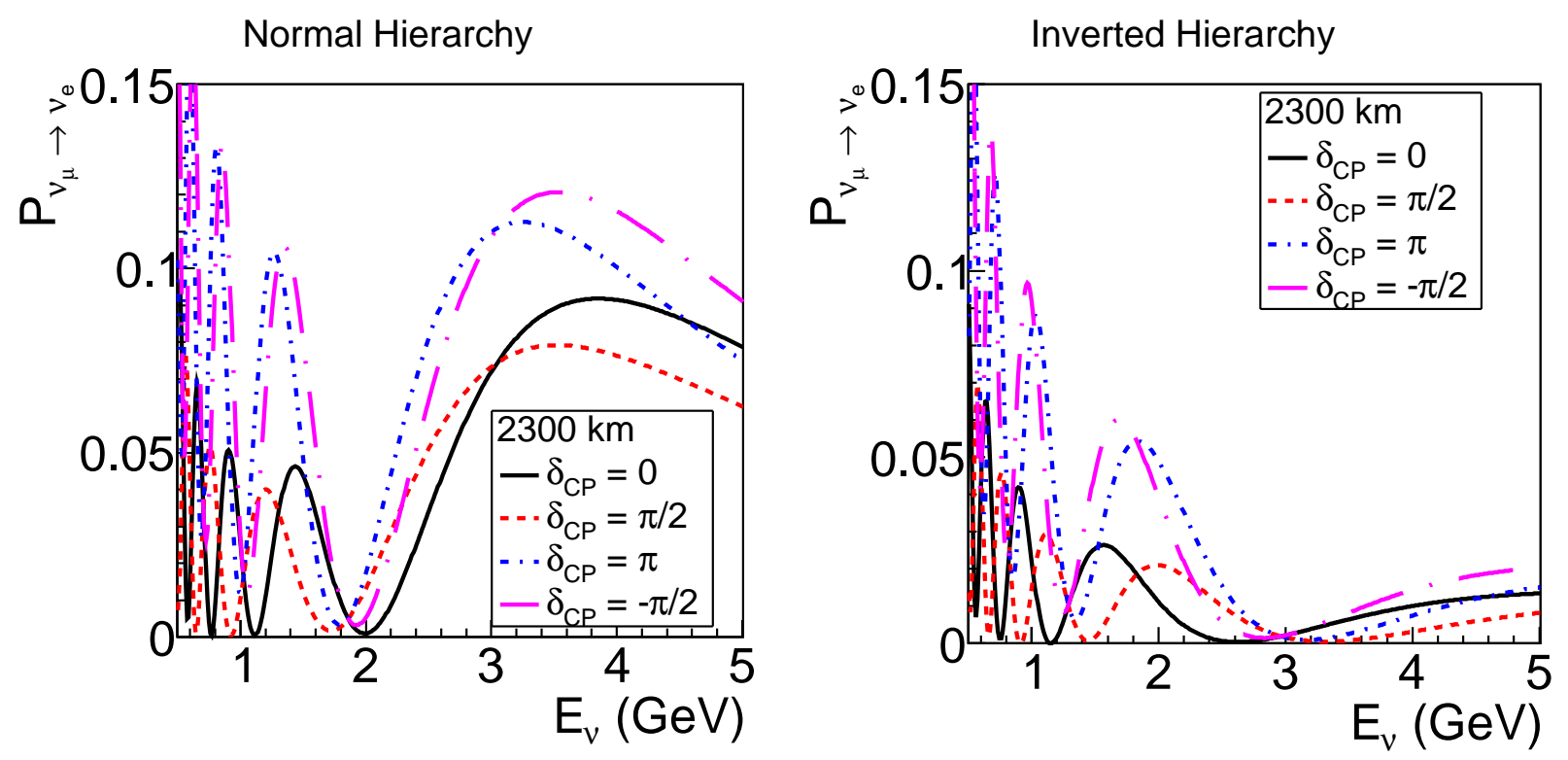

Figure 26: The expected $\nu_{\mu} \rightarrow \nu_{e}$ oscillation patterns in LBNO at a baseline of $2300 \mathrm{~km}$. The matter density $\rho$ and the electron fraction $Y_{e}$ are assumed to be $3.2 \mathrm{~g} / \mathrm{cm}^{3}$ and 0.5 , respectively.

\subsubsection{PINGU, ORCA, INO and Hyper Kamiokande}

As explained in Sec. [2.3, the study of atmospheric neutrinos with very large detectors may be used to determine the neutrino mass hierarchy. The long baseline of the upward going atmospheric neutrinos and the corresponding large matter effect, make this program possible. We briefly describe three proposed experiments that are aiming, among other things, to resolve $\mathrm{MH}$. 
The Precision IceCube Next Generation Upgrade (PINGU) is a proposed new multi megaton array for IceCube upgrade at the South Pole Station. Its primary purpose is the determination of neutrino mass hierarchy using the detection of atmospheric neutrinos. The initial design will have a dense array of 40 strings ( $20 \mathrm{~m}$ of average string spacing) with 60 optical modules each $(5 \mathrm{~m}$ module spacing on the string), located in the Deep Core region of the IceCube detector. This will allow reconstruction of the Cherenkov tracks of events, i.e. of the energy and the zenith angle, for a relatively low energy region, $5-15 \mathrm{GeV}$, where the effect of $\mathrm{MH}$ is most evident. Note that the zenith angle determination is equivalent to the neutrino path length determination. The initial design of the array, and description of the corresponding simulation can be found in Ref. [118]. The PINGU detector will also improve other measurements of neutrino oscillation parameters, in particular of the mixing angle $\theta_{23}$. It will be the world's largest detector of the possible supernova neutrino burst. It will also be able to search for the indirect signal from the WIMP dark matter annihilation or decay in the Sun or Galactic Center. In a favorable scenario, PINGU installation might be completed in 2021 or 2022.

Although the atmospheric flux contains both neutrinos and antineutrinos that are difficult to separate experimentally, as well as both $\nu_{\mu}$ and $\nu_{e}$, the large size of the PINGU detector, and the correspondingly large event statistics, makes it possible to overcome these challenges. At the relatively low energies, most of the secondary particles will be contained in the detector volume. Simulation indicates that $\sim 90 \%$ of the charged-current $\nu_{e}$ and $\nu_{\mu}$ induced processes, and of the neutral current events, can be identified.

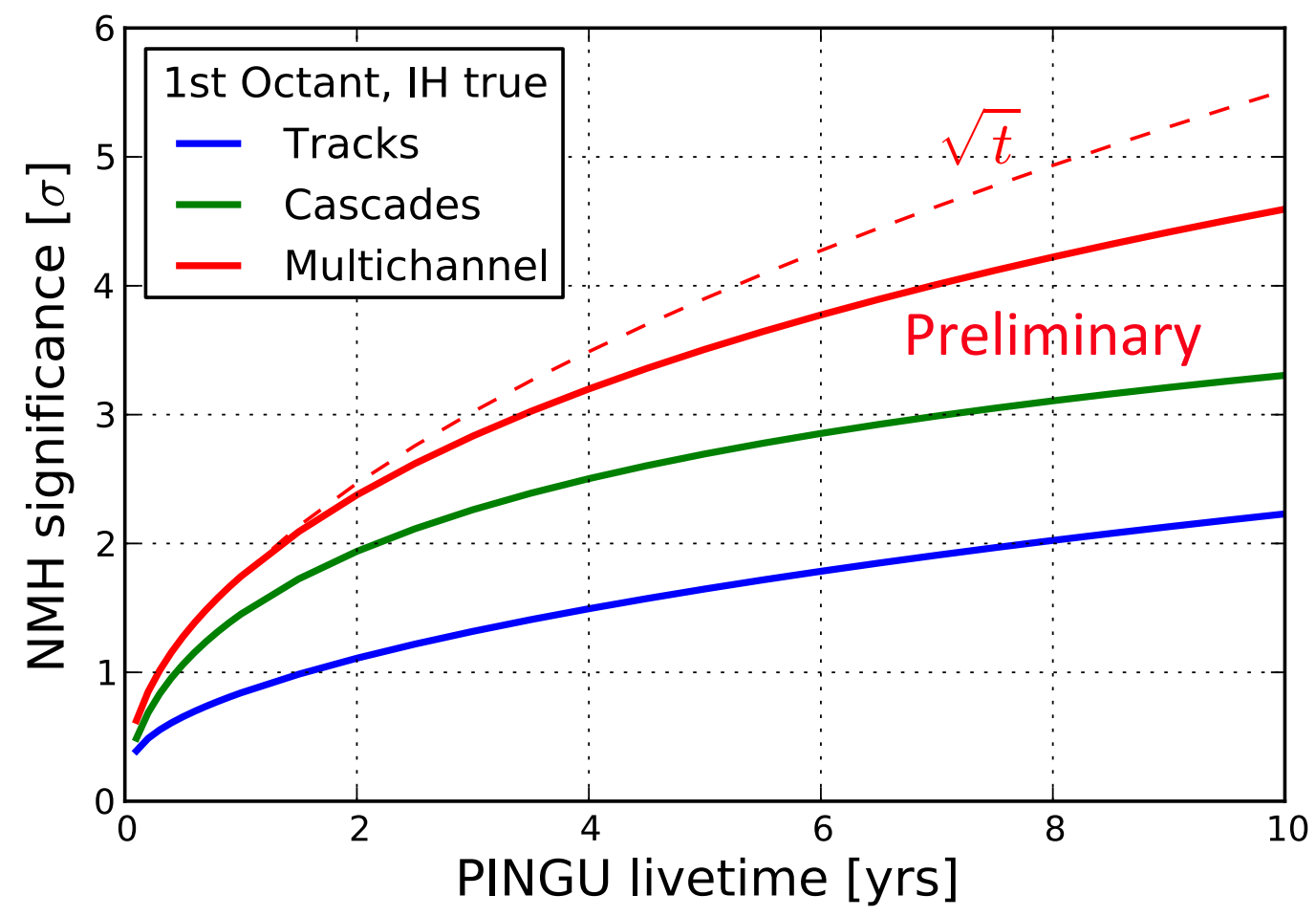

Figure 27: MH sensitivity of PINGU vs. the running time is shown. The blue line (tracks) is based on the identification of the muon track events and the green line is based on the hadronic and electron events. The red line represents the total sensitivity. For comparison the dashed red line show the ideal scaling without systematics. Reproduced with permission from Ref. [118]. 
As shown in Sec. 2.3, it turns out that the survival probabilities for a given $E_{\nu}$ and $\cos \theta_{\text {zenith }}$ for the $\nu_{\mu}$ in the case of $\mathrm{NH}$ is essentially the same as the probability for $\bar{\nu}_{\mu}$ with IH, and vice versa. This appears to be an insurmountable problem. However, the differences in the cross section and kinematics of the $\nu$ and $\bar{\nu}$ interaction with nuclei, as well as the differences in the atmospheric $\nu$ and $\bar{\nu}$ fluxes, makes it possible to separate the two hierarchies. As described in Ref. [11.9], it is possible to identify the "distinguishability" region in the space of $E_{\nu}$ and $\cos \theta_{\text {zenith }}$ where the effect of MH is most pronounced. It is these regions where the most useful information of the MH can be extracted.

Simulation indicates that the PINGU sensitivity to the MH will reach a $3 \sigma$ significance with about 3.5 years of data collection. In Fig. [27, the preliminary plot of the MH significance versus the PINGU detector running time is shown. Note that only when all available information is combined a significant determination of $\mathrm{MH}$ is possible.

ORCA (Oscillation Research with Cosmics in the Abyss) [120] is a proposed option aiming to determine the MH using the deep-sea neutrino telescope technology developed for the KM3NeT project (A multi-km³ sized Neutrino Telescope) [121]. The experimental principle of ORCA is similar to that of PINGU. Instead of deep ice in the south pole, ORCA is expected to deploy large 3-dimensional arrays of photo-sensors to detect Cherenkov lights in the deep Mediterranean Sea. A 3-5 $\sigma$ MH sensitivity is targeted for a $\sim 20$ Mton·year overall exposure given the neutrino detecting threshold around $5 \mathrm{GeV}$.

The India-based Neutrino Observatory (INO) [122] is a planned underground experiment at Pottipuram, Theni (India) to study the atmospheric neutrinos. The project has been approved by the Indian government with the tentative start of data taking by 2020. The proposed detector is a magnetized iron calorimeter with 52 kiloton mass and 1.5 Tesla magnetic field, divided into three detector modules. Each module contains 151 layers of $5.6 \mathrm{~cm}$ iron plates with the resistive plate chambers (RPC) as active detector elements in the gaps between the plates. INO will have excellent muon energy and direction resolutions as well as a charge reconstruction capability. Besides $\mathrm{MH}$, INO will be also sensitive to $\theta_{23}$ and $\Delta m_{32}^{2}$. Fig. [28 shows the projected MH sensitivity of INO vs. data taking time [123]. Note that the $\mathrm{MH}$ sensitivity strongly depends on the true values of $\theta_{23}$ and $\theta_{13}$ which, however, might be precisely known by the time the detector begins data taking. The MH sensitivity is significantly larger if the mixing angle $\theta_{23}$ is in the second octant, i.e. if $\sin ^{2} \theta_{23}>0.5$. The same also applies to MH sensitivity in PINGU. 

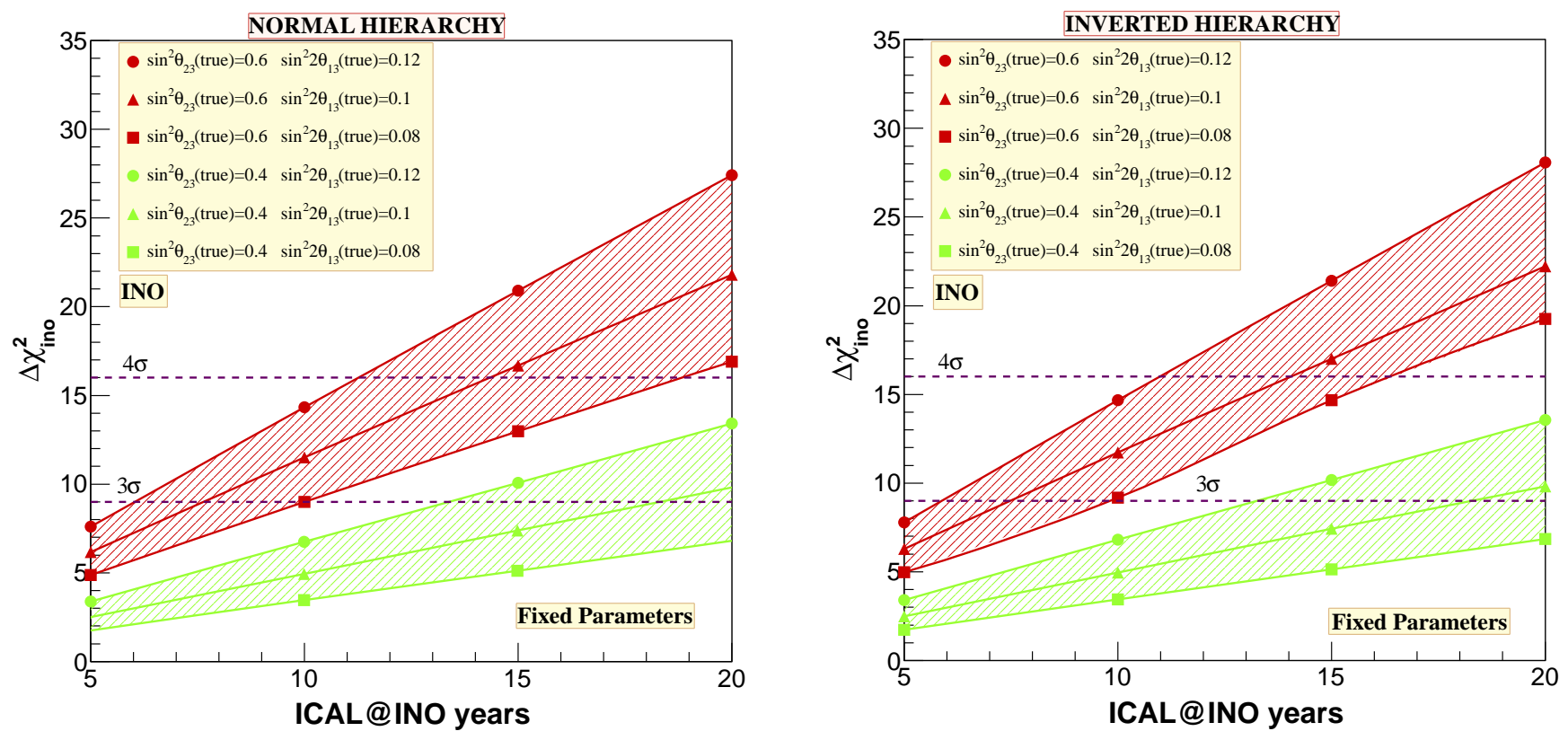

Figure 28: MH sensitivities of INO with respect to the running time are shown as $\Delta \chi_{i n o}^{2}:=\overline{\Delta T}$. Left and right panels assume the true $\mathrm{MH}$ is normal and inverted, respectively. Different lines represent different values of $\sin ^{2} \theta_{23}$ and $\sin ^{2} 2 \theta_{13}$. During the analysis, all the oscillation parameters are fixed at their input true values. Reproduced with permission from Ref. [12:3].

Hyper-Kamiokande [124] is a proposed experiment near the site of the previous Kamiokande and the current Super-Kamiokande detectors in Japan. The master plan at present suggests that the construction and the operation might begin in 2018 and 2025, respectively. The detector technology will be water Cherenkov similar as in Super-Kamiokande, only many times larger, with a total mass of 1 Megatons (560 kiloton fiducial mass). While the main experimental program at Hyper-Kamiokande is the precision measurement of $\nu_{\mu} \rightarrow \nu_{e}$ and $\bar{\nu}_{\mu} \rightarrow \bar{\nu}_{e}$ oscillations with accelerator neutrinos at a relative short baseline $(295 \mathrm{~km})$ to search for $\mathrm{CP}$ violation in the lepton sector, Hyper Kamiokande is also sensitive to $\mathrm{MH}$ with atmospheric neutrinos. Fig. $2 . \mathrm{g}$ shows the atmospheric neutrino MH sensitivity with ten years of data taking. Again, the MH sensitivity is significantly larger if the mixing angle $\theta_{23}$ happens to be in the second octant. 


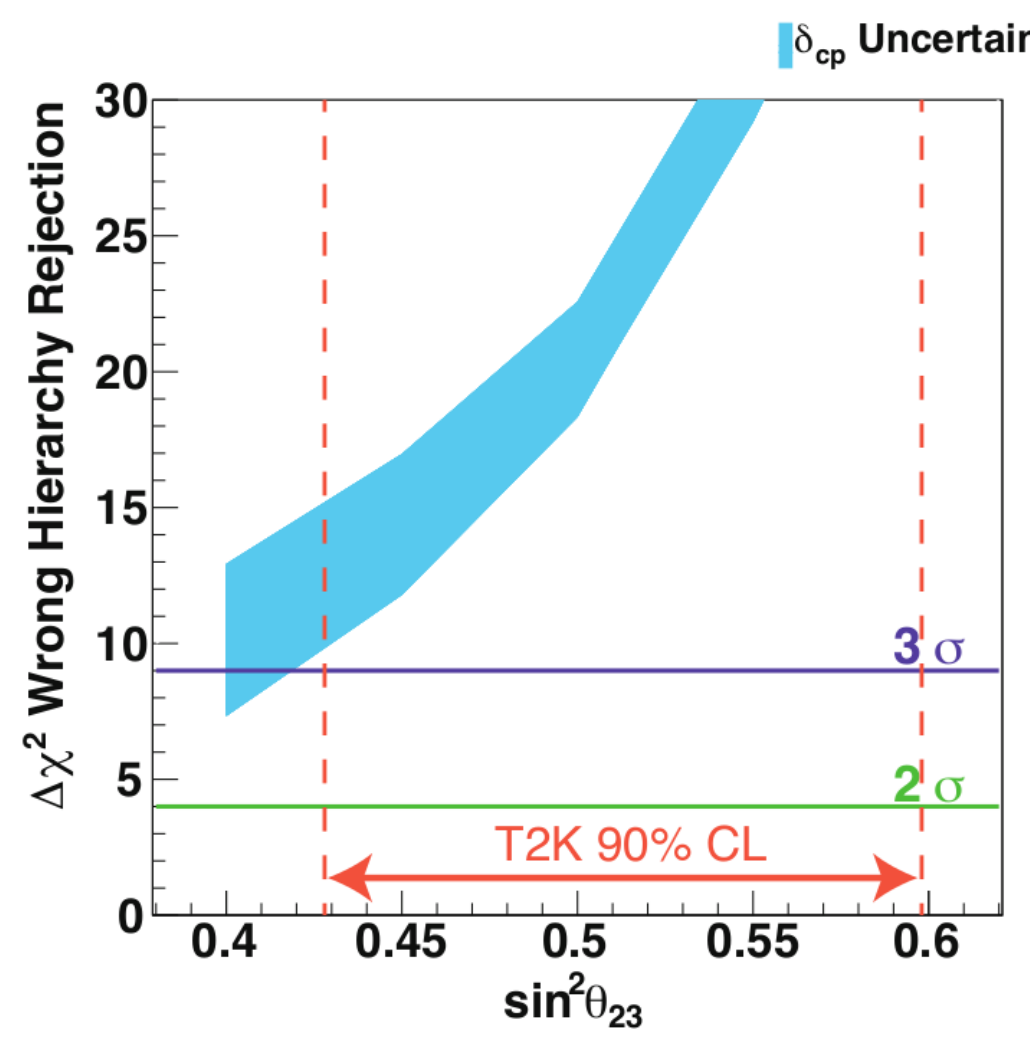

Figure 29: The MH sensitivity of Hyper-Kamiokande with respect to the true value of $\sin ^{2} \theta_{23}$ is shown as $\Delta \chi^{2}:=\overline{\Delta T}$. The blue band represents the uncertainty of the currently unknown CP phase $\delta_{C P}$. A ten-years exposure is assumed. Reproduced with permission from Ref. [124].

\subsubsection{Medium baseline reactor experiments: JUNO and RENO-50}

As described in the Sec. 2.4, reactor experiments at baselines $\approx 60 \mathrm{~km}$ are well suited for the MH determination. Two such experiments have been proposed and are currently at various stages of preparation and construction. Here we describe them in more details, using the JUNO experiment as the main example.

Jiangmen Underground Neutrino Observatory (JUNO) is a reactor neutrino experiment under construction in Jiangmen City, Guangdong Province, China [125, 126]. The groundbreaking for JUNO started in January 2015. The plan calls for the construction to last until 2017, assembly and installation in 2018-2019 and start of the data taking in 2020. Fig. 30 shows the JUNO site. The JUNO detector will mostly receive $\bar{\nu}_{e}$ from two reactor complexes at Taishan and Yangjiang. The Taishan reactor complex contains six reactor cores with a total thermal power of $17.4 \mathrm{GW}$. The Yangjiang reactor complex has two reactor cores with a total thermal power of $9.2 \mathrm{GW}$. There are two additional reactor cores $(9.2 \mathrm{GW})$ planned at the Yangjiang site. The average baseline of JUNO is $\sim 52.5 \mathrm{~km}$ with a RMS (root mean square) of $0.25 \mathrm{~km}$.

The central detector of JUNO is a 20 kton liquid scintillator (LS) detector with a total overburden of 1850 meter water equivalent. Figure 10 shows a conceptual design of the spherical LS central detector. The photo-cathode coverage is expected to reach 75\%. Together with high performance LS (high photon yield with $>14,000$ photons per $\mathrm{MeV}$, optical attenuation length of $30 \mathrm{~m}$ or better) and high quantum efficiency PMTs (peak quantum efficiency reaching 35\%), JUNO aims to achieve an energy resolution of better than $1.9 \%$ at $2.5 \mathrm{MeV}$, which is essential for the $\mathrm{MH}$ determination. This level of energy resolution requires a comprehensive calibration system to minimize position dependent corrections to the reconstructed energy. The spherical central detector will be placed inside a water pool instrumented 
with PMTs to identify and veto cosmic muons and provide shielding from radioactive backgrounds in the underground environment. A muon tracker on top of the detector will further enhance the muon identification.

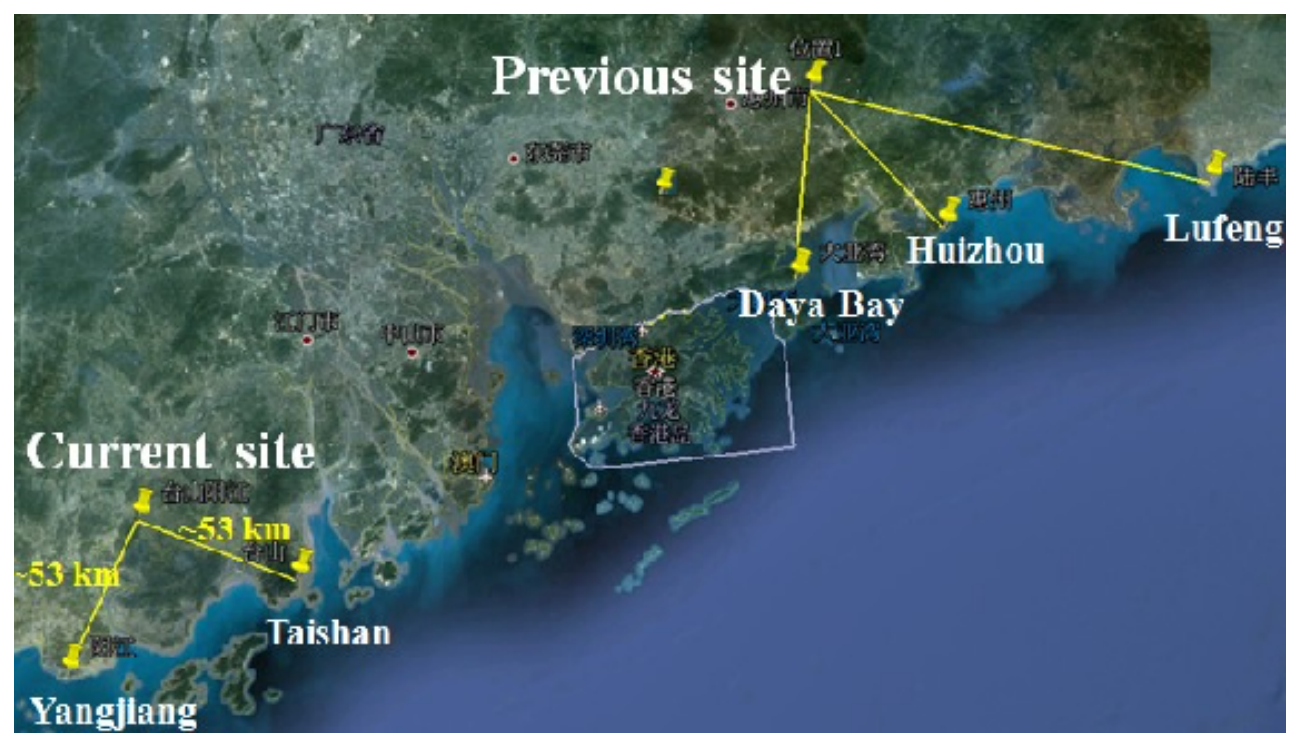

Figure 30: Experimental site of JUNO. Hongkong and Daya Bay reactor complex are labeled. Reproduced with the permission from Ref. [125].

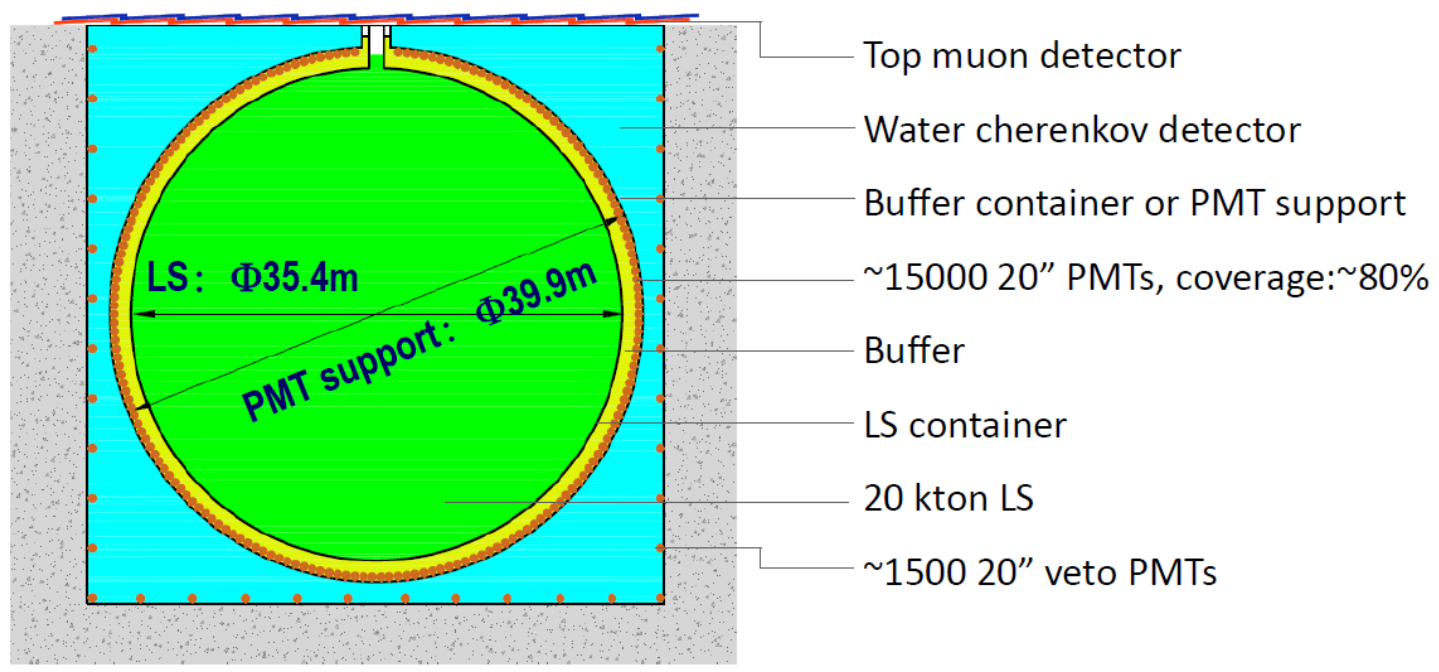

Figure 31: One conceptual design of JUNO detector. Reproduced with the permission from Ref. [125].

RENO-50 is a proposed large (18 kton) liquid scintillator detector in Korea [127]. At the present time, the schedule for the facility and detector construction aims at years 2015-2020 with the start of operation in 2021. The central detector is expected to be equipped with $15 \mathrm{k}$ high quantum efficiency 20inch PMTs. Fig. 32 shows the proposed site of RENO-50, roughly $50 \mathrm{~km}$ away from the Hanbit nuclear power plant. The physics goal of RENO-50 is similar to those of JUNO, including the determination of $\mathrm{MH}$ and the precision measurements of neutrino mixings. In order to reach the desired energy resolution for $\mathrm{MH}$ determination, RENO-50 is carrying out R\&D to i) enhance the light yield of liquid scintillator from $\sim 500$ to $>1000$ photoelectrons/MeV, ii) increase the attenuation length of the liquid scinillator from $\sim 15$ to $\sim 25 \mathrm{~m}$, and iii) enhance the quantum efficiency of PMT from $20 \%$ to $35 \%$. Additional $\mathrm{R} \& \mathrm{D}$ include the reduction of liquid scintillator radioactivity to $10^{-16} \mathrm{~g} / \mathrm{g}$ for $\mathrm{U}$ and $\mathrm{Th}$. 


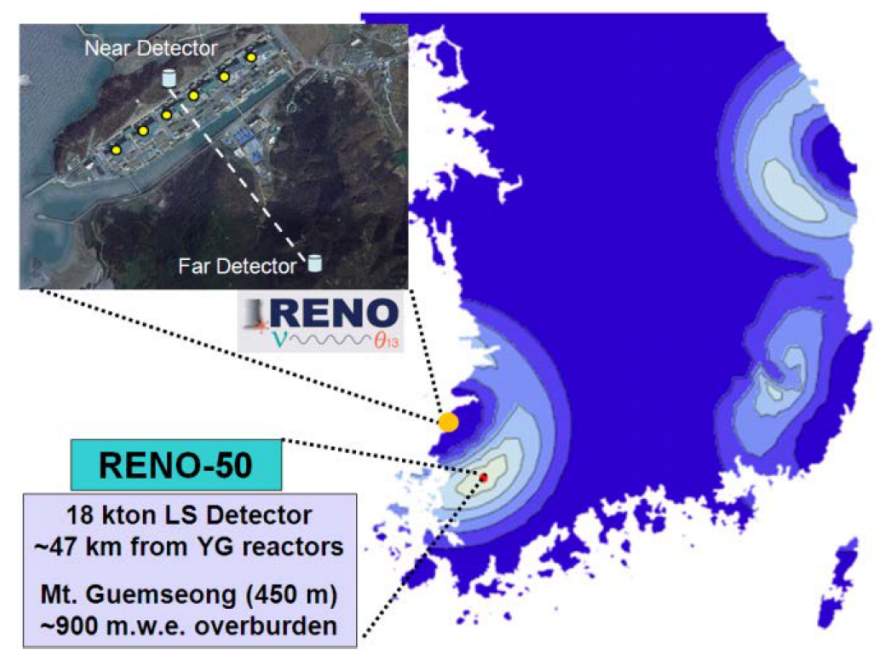

Figure 32: The RENO-50 detector will be located at underground of Mt. Guemseong in a city of Naju, $47 \mathrm{~km}$ from the Hanbit nuclear power plant. Reproduced with the permission from Ref. [127].

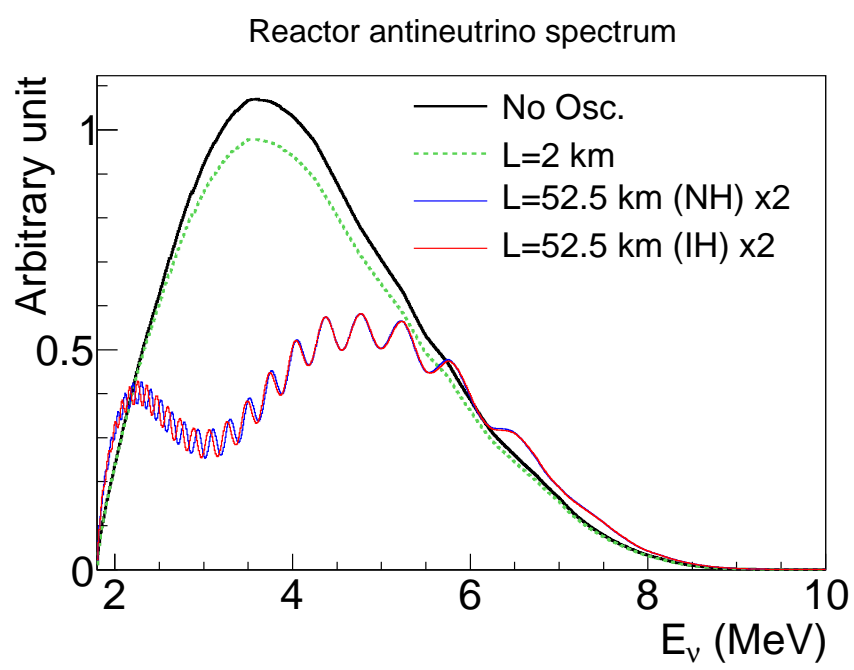

Figure 33: The spectral distortion at JUNO (arbitrary scale in the vertical axis) for both normal and inverted hierarchies with a perfect energy resolution. $\Delta m_{32}^{2}$ is assumed to be $2.41 \times 10^{-3} \mathrm{eV}^{2}$ and $-2.53 \times 10^{-3} \mathrm{eV}^{2}$ for $\mathrm{NH}$ and $\mathrm{IH}$, respectively. The reactor spectrum without oscillation and the oscillated spectrum at $2 \mathrm{~km}$ are shown for comparison.

The principle of the $\mathrm{MH}$ determination in a medium baseline reactor neutrino experiment has been illustrated in Sec. W. Figure B3.3 shows the distortions of the ideal spectrum at JUNO for both normal and inverted $\mathrm{MH}$ with the perfect energy resolution. The effective mass-squared splitting $\Delta m_{e e}^{2}$ (fast oscillation frequency) at low energy (2-4 MeV) is higher than that at high energy (4-8 MeV) for $\mathrm{NH}$, and vice versa for $\mathrm{IH}$. As the oscillation at the low energy region (2-4 MeV) is very rapid, a good energy resolution is required to extract the oscillation signal. In general, a better than $3 \% / \sqrt{E(\mathrm{MeV})}$ energy resolution has been discussed in the literature as the requirement. In reality, the energy resolution is generally parameterized as:

$$
\frac{\Delta E}{E}=\sqrt{a+\frac{b}{E}+\frac{c}{E^{2}}} .
$$

Here, the $1 / E$ term represents the contribution from photon statistics; the $1 / E^{2}$ term represents the contribution from PMT dark noise; the constant term represents the contribution from residual non- 
uniformity after calibration. As the $\mathrm{MH}$ signal is around $2.5 \mathrm{MeV}$, the requirement on the energy resolution is naturally converted to better than $1.9 \%$ at $2.5 \mathrm{MeV}$ prompt energy. Beside the energy resolution, a $\ll 1 \%$ absolute energy scale uncertainty [69, [28] is also required in order to unambiguously extract the MH signal. Such a requirement can be addressed with a dedicated calibration system with a small positron accelerator (such as a Pelletron).
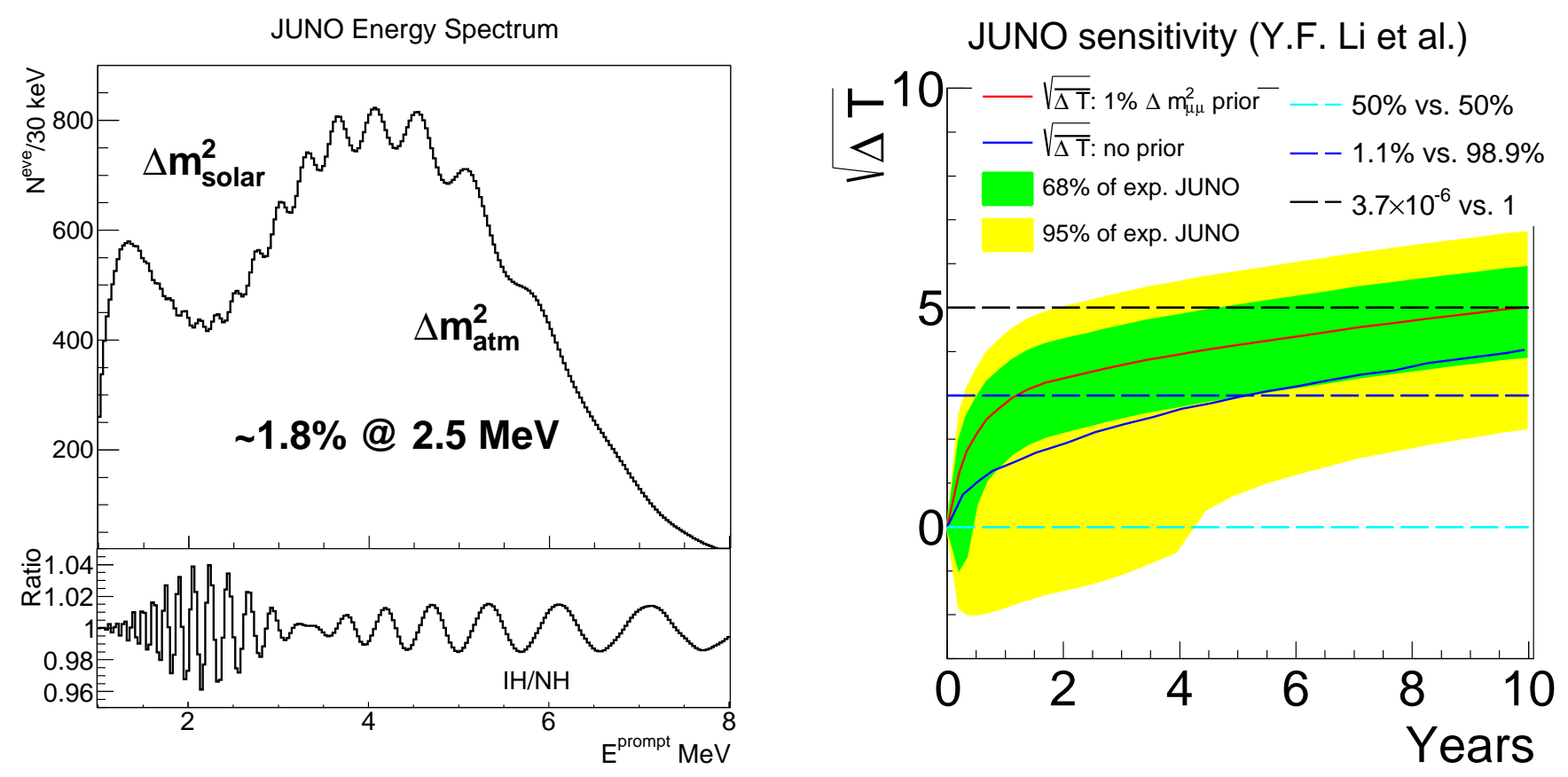

Figure 34: (Left) The expected prompt energy spectrum of JUNO for six years of data taking with a $20 \mathrm{kt}$ detector and $36 \mathrm{GW}_{t h}$ reactor power (a total of 100k IBD events). The energy resolution is assumed to be about $1.8 \%$ at $2.5 \mathrm{MeV}$. The bottom panel shows the $\mathrm{MH}$ signal as the ratio of spectrum of inverted hierarchy over the normal hierarchy. (Right) JUNO's sensitivity evolution with respect to calendar years. [ [ [2] ]. The $\Delta T$ is a test statistics consisting likelihoods of normal and inverted $\mathrm{MH}$ for data $x$. The green and yellow bands represent the $68 \%$ and $95 \%$ expectations, respectively, taking into account the fluctuations in statistics and variations in systematic. The dashed lines correspond to the probability ratios of the normal vs. inverted $\mathrm{MH}$ in the Bayesian framework.

In addition to MH, JUNO will perform precision measurements of the neutrino mixing, which is a powerful tool to test the standard 3-flavor neutrino framework (or $\nu \mathrm{SM}$ ). The precision measurement of $\sin ^{2} 2 \theta_{12}$ will i) lay the foundation for a future sub-1\% direct unitarity test of the PMNS matrix [129, 41], ii) constrain the allowed region of the effective neutrino mass associated with the decay rate of the neutrinoless double beta decay, and iii) test various phenomenological models of neutrino masses and mixing [130], such as $\theta_{12}=35^{\circ}+\theta_{13} \cos \delta_{C P}, \theta_{12}=32^{\circ}+\theta_{13} \cos \delta_{C P}$, and $\theta_{12}=45+\theta_{13} \cos \delta_{C P}$, with $\delta_{C P}$ being the CP phase in PMNS matrix. The precision measurement of $\Delta m_{e e}^{2}$ (or $\left.\Delta m_{32}^{2}\right)$ will i) test an important sum rule $\Delta m_{13}^{2}+\Delta m_{21}^{2}+\Delta m_{32}^{2}=0$ and ii) reveal additional information regarding $\mathrm{MH}$, when combined with the precision $\Delta m_{\mu \mu}^{2}$ measurements from muon neutrino and antineutrino disappearance in accelerator experiments. JUNO's expected precision of $\Delta m_{21}^{2}, \Delta m_{32}^{2}$, and $\sin ^{2} 2 \theta_{21}$ will be about $\sim 0.6 \%$ [128]. These precision measurements are complementary to the atmospheric and long-baseline accelerator neutrino programs. Together, the next-generation reactor neutrino and accelerator neutrino experiments will access all seven neutrino mixing parameters governing the oscillation phenomenon within the three-neutrino framework.

To summarize, the major physics goals of the next generation medium baseline reactor experiments such as JUNO and RENO-50 are: 
- to be the first experiment to simultaneously observe neutrino oscillations from both the atmospheric and the solar neutrino mass-squared splittings (see Fig. B4),

- to be the first experiment to observe more than two oscillation cycles of the atmospheric masssquared splitting (see Fig. 34),

- determination of the neutrino mass hierarchy, i.e. whether $\Delta m_{32}^{2}$ is positive or negative, through the precision measurement of the spectral distortion. Right panel of Fig. B4 shows the expected sensitivity of JUNO [[?2] vs. the running time.

- precision measurements of $\sin ^{2} 2 \theta_{21}, \Delta m_{32}^{2}$, and $\Delta m_{21}^{2}$ to better than $1 \%$. We should note that the precision measurement of $\Delta m_{32}^{2}$ requires the knowledge of the neutrino mass hierarchy.

Besides these, the $20 \mathrm{kt}$ underground liquid scintillator detector offers a rich physics program including the search for proton decay, geoneutrinos, supernova neutrinos, and many exotic neutrino physics topics.

\section{Summary and Outlook}

In this work we review the theoretical and experimental issues one has to resolve in order to determine the neutrino mass hierarchy. The motivations and the theoretical framework are depicted in Sec. 四. Then, in Sec. $\nabla$ the practical methods that can be used in the MH determination are described. Finally, in Sec. B] the various existing and proposed or planned experiments that have the capacity to shed light on the $\mathrm{MH}$ are briefly reviewed.

Determining the neutrino mass hierarchy is a challenging problem. Even though one could naively expect that the difference between the normal and inverted hierarchies, the two possible mass ordering shown in Fig. $\mathbb{1}$, is going to be significant, in fact the difference is rather subtle in the real data. That is so because the majority of experimental findings, at least for the most common case of neutrinos propagating in vacuum, can be described in a reasonable approximation as stemming from mixing of just two neutrino states; in that case the oscillation pattern depends only on the absolute values of the mass-squared differences, not on their sign. Thus, in order to determine the MH, i.e. the sign of $\Delta m_{3 x}^{2}$, it is necessary to go beyond that approximation and consider the effects associated with mixing of the three neutrino states. The corresponding effects are, however, suppressed by the two small parameters present in the oscillation formalism, the mixing angle $\theta_{13} \sim 8.5^{\circ}$ and the mass square difference ratio $\Delta m_{21}^{2} / \Delta m_{32}^{2} \sim 1 / 30$. The fact that it is possible to approach the problem in this way at all is the result of successful experiments that have recently shown that the mixing angle $\theta_{13}$ is not as small as many people believed. That finding opens the way to the MH determination. At the same time, that finding also shows that the seemingly simple and attractive possibility of the so-called tribimaximal mixing matrix, originally suggested in Ref. [1:31] and discussed in many papers since, is not realized in nature. Even though the PMNS matrix, as determined from the oscillation experiments, looks similar to the simple form suggested, with $\left|U_{i j}\right|^{2}$ expressed in rational numbers $2 / 3,1 / 2,1 / 3,1 / 6$ and 0 , the reality is more complicated.

The other way to approach the MH is to use the neutrino propagation in matter, with the additional interaction that depends on the mass ordering. The mass ordering of neutrinos $\nu_{1}$ and $\nu_{2}\left(m_{\nu_{1}}<m_{\nu_{2}}\right)$ is determined in this way thanks to the results of the solar neutrino experiments. Solar neutrinos propagate from the central region of the Sun, over large distance with high electron density, so the matter effects are very pronounced. However, the corresponding oscillation length $L_{\text {matter }}$ to determine the mass ordering of neutrinos $\nu_{1}$ and $\nu_{3}$ (see Eq. (田) is very long $\mathrm{O}\left(10^{3}\right) \mathrm{km}$ for the typical terrestrial electron densities, so the practical application requires very intense neutrino sources and large and complicated detectors. 
Instead of neutrinos from accelerators, one could use the atmospheric neutrinos that come from all directions, including the upward going ones that traverse the Earth diameter. In that case the matter effect is large, but the MH determination is hindered by the fact that the atmospheric neutrino flux contains both $\nu_{\mu}$ and $\nu_{e}$ and also both $\nu$ and $\bar{\nu}$ and thus one needs a way to recognize the flavor and polarity of the interaction products, or employ the fact that the different components of the atmospheric neutrino flux have different intensities and different interaction cross sections.

Having enumerated the difficulties we can understand why the $\mathrm{MH}$ remains unknown. But that situation can change. As discussed in Sec. $\mathbb{Z}$ one can approach the MH problem with neutrinos from accelerators and from the atmosphere through the matter effect, or from nuclear reactors through exploring difference between $\Delta m_{32}^{2}$ and $\Delta m_{31}^{2}$. Since the effects are subtle, it is necessary to use adequate devices and reliable statistical analysis methods. Also, it would be desirable if all, or at least several, of the possible methods were used in practice. The results would be complementary and increase the confidence that the conclusions are indeed correct.

While some of the existing experiments, described in Sec. B, have a chance to resolve the MH problem soon, it is likely that eventually the more complex and costly devices described there will be needed. Again, complementarity will be desirable and very useful. Realistically, some of the listed experiments might begin operation within this decade, with results after few years of operation. So stay tuned. Rewards are coming only to those who are patient.

\section{Acknowledgments}

We thank Brett Viren, Chao Zhang, Steve Kettell, Wei Tang for reading the manuscript. This material is support in part by the U.S. Department of Energy, Office of Science, Office of High Energy Physics, Early Career Research program under contract number DE-SC0012704.

\section{References}

[1] B. Pontecorvo. Sov. Phys. JETP, 6:429, 1957.

[2] B. Pontecorvo. Sov. Phys. JETP, 26:984, 1968.

[3] Z. Maki, M. Nakagawa, and S. Sakata. Prog. Theor. Phys., 28:870, 1962.

[4] M.C. Gonzalez-Garcia, Michele Maltoni, Jordi Salvado, and Thomas Schwetz. Global fit to three neutrino mixing: critical look at present precision. JHEP, 1212:123, 2012.

[5] Neutrino 2014: the 24th Interactional Conference on Neutrino Physics and Astrophysics, June 2-7th, 2014, Boston, USA. http://neutrino2014.bu.edu/program/.

[6] D.V. Forero, M. Tortola, and J.W.F. Valle. Neutrino oscillations refitted. 2014.

[7] K.A. Olive et al. Review of Particle Physics. Chin.Phys., C38:090001, 2014.

[8] L. Wolfenstein. Neutrino oscillations in matter. Phys. Rev., D17:2369, 1978.

[9] S. P. Mikheev and A. Yu. Smirnov. Resonant amplification of neutrino oscillations in matter and solar neutrino spectroscopy. Nuovo Cim., C9:17-26, 1986.

[10] S. P. Mikheev and A. Yu. Smirnov. Resonance enhancement of oscillations in matter and solar neutrino spectroscopy. Sov. J. Nucl. Phys., 42:913-917, 1985. 
[11] M.V. Chizhov and S.T. Petcov. New conditions for a total neutrino conversion in a medium. Phys.Rev.Lett., 83:1096-1099, 1999.

[12] Evgeny K. Akhmedov and A. Yu. Smirnov. Comment on 'New conditions for a total neutrino conversion in a medium'. Phys.Rev.Lett., 85:3978, 2000.

[13] Huaiyu Duan, George M. Fuller, and Yong-Zhong Qian. Collective Neutrino Oscillations. Ann.Rev.Nucl.Part.Sci., 60:569-594, 2010.

[14] Georg G. Raffelt. Physics opportunities with supernova neutrinos. Prog.Part.Nucl.Phys., 64:393399, 2010.

[15] M. Apollonio et al. Limits on neutrino oscillations from the CHOOZ experiment. Phys.Lett., B466:415-430, 1999.

[16] M. Apollonio et al. Search for neutrino oscillations on a long baseline at the CHOOZ nuclear power station. Eur.Phys.J., C27:331-374, 2003.

[17] F. Boehm, J. Busenitz, B. Cook, G. Gratta, H. Henrikson, et al. Final results from the Palo Verde neutrino oscillation experiment. Phys.Rev., D64:112001, 2001.

[18] G.L. Fogli, E. Lisi, A. Marrone, A. Palazzo, and A.M. Rotunno. Evidence of $\theta_{13}>0$ from global neutrino data analysis. Phys.Rev., D84:053007, 2011.

[19] P. Adamson et al. Improved search for muon-neutrino to electron-neutrino oscillations in MINOS. Phys.Rev.Lett., 107:181802, 2011.

[20] K. Abe et al. Indication of Electron Neutrino Appearance from an Accelerator-produced Off-axis Muon Neutrino Beam. Phys.Rev.Lett., 107:041801, 2011.

[21] Y. Abe et al. Indication for the disappearance of reactor electron antineutrinos in the Double Chooz experiment. Phys.Rev.Lett., 108:131801, 2012.

[22] F.P. An et al. Observation of electron-antineutrino disappearance at Daya Bay. Phys.Rev.Lett., 108:171803, 2012.

[23] J.K. Ahn et al. Observation of Reactor Electron Antineutrino Disappearance in the RENO Experiment. Phys.Rev.Lett., 108:191802, 2012.

[24] F.P. An et al. Improved Measurement of Electron Antineutrino Disappearance at Daya Bay. Chin.Phys., C37:011001, 2013.

[25] Justin Evans. The MINOS experiment: results and prospects. Adv.High Energy Phys., 2013:182537, 2013.

[26] K. Abe et al. Observation of Electron Neutrino Appearance in a Muon Neutrino Beam. Phys.Rev.Lett., 112:061802, 2014.

[27] Y. Abe et al. First Measurement of $\theta_{13}$ from Delayed Neutron Capture on Hydrogen in the Double Chooz Experiment. Phys.Lett., B723:66-70, 2013.

[28] S.-H. Seo. New Results from RENO. ArXiv e-prints, December 2013.

[29] F.P. An et al. Independent Measurement of Theta13 via Neutron Capture on Hydrogen at Daya Bay. Phys.Rev., D90:071101, 2014. 
[30] C. Zhang (Daya Bay), Neutrino 2014, https://indico.fnal.gov/getFile.py/access? contribId=2.56\&.session $I d=15 \&$.res $T d=0$ \&material $T d=s]$ ides\&.conf $T d=8022$.

[31] Daya Bay Collaboration. U.S. P5 contribution, http://www.usparticlephysics.org/sites/ default/files/webform/p5/DayaBay_RunPlan_0.pdf (2013).

[32] G. Danby, J.M. Gaillard, Konstantin A. Goulianos, L.M. Lederman, Nari B. Mistry, et al. Observation of High-Energy Neutrino Reactions and the Existence of Two Kinds of Neutrinos. Phys.Rev.Lett., 9:36-44, 1962.

[33] K. Kodama et al. Observation of tau neutrino interactions. Phys.Lett., B504:218-224, 2001.

[34] M.H. Ahn et al. Measurement of Neutrino Oscillation by the K2K Experiment. Phys.Rev., D74:072003, 2006.

[35] N. Agafonova et al. Evidence for $\nu_{\mu} \rightarrow \nu_{\tau}$ appearance in the cngs neutrino beam with the opera experiment. Phys.Rev., D89:051102, 2014.

[36] P. Adamson et al. Combined analysis of $\nu_{\mu}$ disappearance and $\nu_{\mu} \rightarrow \nu_{e}$ appearance in MINOS using accelerator and atmospheric neutrinos. Phys.Rev.Lett., 112:191801, 2014.

[37] F.P. An et al. Observation of electron-antineutrino disappearance at Daya Bay. Phys.Rev.Lett., 108:171803, 2012.

[38] F.P. An et al. Spectral measurement of electron antineutrino oscillation amplitude and frequency at Daya Bay. Phys.Rev.Lett., 112:061801, 2014.

[39] J.K. Ahn et al. Observation of Reactor Electron Antineutrino Disappearance in the RENO Experiment. Phys.Rev.Lett., 108:191802, 2012.

[40] Y. Abe et al. Reactor electron antineutrino disappearance in the Double Chooz experiment. Phys.Rev., D86:052008, 2012.

[41] X. Qian, C. Zhang, M. Diwan, and P. Vogel. Unitarity Tests of the Neutrino Mixing Matrix. 2013.

[42] D. Beavis et al. Long baseline neutrino oscillation experiment at the ags. 1995.

[43] A.A. Aguilar-Arevalo et al. The Neutrino Flux prediction at MiniBooNE. Phys.Rev., D79:072002, 2009.

[44] J.A. Formaggio and G.P. Zeller. From eV to EeV: Neutrino Cross Sections Across Energy Scales. Rev.Mod.Phys., 84:1307, 2012.

[45] Y. Fukuda et al. Evidence for oscillation of atmospheric neutrinos. Phys.Rev.Lett., 81:1562-1567, 1998.

[46] M. Honda, T. Kajita, K. Kasahara, and S. Midorikawa. Improvement of low energy atmospheric neutrino flux calculation using the JAM nuclear interaction model. Phys.Rev., D83:123001, 2011.

[47] Morihiro Honda, T. Kajita, K. Kasahara, S. Midorikawa, and T. Sanuki. Calculation of atmospheric neutrino flux using the interaction model calibrated with atmospheric muon data. Phys.Rev., D75:043006, 2007. 
[48] G.D. Barr, T.K. Gaisser, P. Lipari, Simon Robbins, and T. Stanev. A Three - dimensional calculation of atmospheric neutrinos. Phys.Rev., D70:023006, 2004.

[49] G. Battistoni, A. Ferrari, T. Montaruli, and P.R. Sala. The FLUKA atmospheric neutrino flux calculation. Astropart.Phys., 19:269-290, 2003.

[50] D. Franco, C. Jollet, A. Kouchner, V. Kulikovskiy, A. Meregaglia, et al. Mass hierarchy discrimination with atmospheric neutrinos in large volume ice/water Cherenkov detectors. JHEP, 1304:008, 2013.

[51] Mathieu Ribordy and Alexei Yu Smirnov. Improving the neutrino mass hierarchy identification with inelasticity measurement in PINGU and ORCA. Phys.Rev., D87(11):113007, 2013.

[52] Walter Winter. Neutrino mass hierarchy determination with IceCube-PINGU. Phys.Rev., D88(1):013013, 2013.

[53] Shao-Feng Ge and Kaoru Hagiwara. Physics Reach of Atmospheric Neutrino Measurements at PINGU. JHEP, 1409:024, 2014.

[54] F. Capozzi, E. Lisi, and A. Marrone. PINGU and the neutrino mass hierarchy: Statistical and systematic aspects. Phys.Rev., D91(7):073011, 2015.

[55] A. M. Dziewonski and D. L. Anderson. Preliminary reference Earth model. Physics of the Earth and Planetary Interiors, 25(4):297-356, 1981.

[56] Brett Viren. https://github.com/brettviren/nuosc.

[57] C.L. Cowan, F. Reines, F.B. Harrison, H.W. Kruse, and A.D. McGuire. Detection of the free neutrino: A Confirmation. Science, 124:103-104, 1956.

[58] T. Araki et al. Measurement of neutrino oscillation with KamLAND: Evidence of spectral distortion. Phys.Rev.Lett., 94:081801, 2005.

[59] K. Schreckenbach, G. Colvin, W. Gelletly, and F. Von Feilitzsch. DETERMINATION OF THE ANTI-NEUTRINO SPECTRUM FROM U-235 THERMAL NEUTRON FISSION PRODUCTS UP TO 9.5-MEV. Phys.Lett., B160:325-330, 1985.

[60] P. Vogel, G.K. Schenter, F.M. Mann, and R.E. Schenter. Reactor Anti-neutrino Spectra and Their Application to Anti-neutrino Induced Reactions. 2. Phys.Rev., C24:1543-1553, 1981.

[61] A.A. Hahn, K. Schreckenbach, G. Colvin, B. Krusche, W. Gelletly, et al. Anti-neutrino Spectra From ${ }^{241} \mathrm{Pu}$ and ${ }^{239} \mathrm{Pu}$ Thermal Neutron Fission Products. Phys.Lett., B218:365-368, 1989.

[62] Xin Qian and Wei Wang. Reactor neutrino experiments: $\theta_{13}$ and beyond. Mod.Phys.Lett., A29:1430016, 2014.

[63] S.T. Petcov and M. Piai. The LMA MSW solution of the solar neutrino problem, inverted neutrino mass hierarchy and reactor neutrino experiments. Phys.Lett., B533:94-106, 2002.

[64] John Learned, Stephen T. Dye, Sandip Pakvasa, and Robert C. Svoboda. Determination of neutrino mass hierarchy and $\theta_{13}$ with a remote detector of reactor antineutrinos. Phys.Rev., D78:071302, 2008. 
[65] H. Minakata, H. Nunokawa, Stephen J. Parke, and R. Zukanovich Funchal. Determining neutrino mass hierarchy by precision measurements in electron and muon neutrino disappearance experiments. Phys.Rev., D74:053008, 2006.

[66] Liang Zhan, Yifang Wang, Jun Cao, and Liangjian Wen. Determination of the Neutrino Mass Hierarchy at an Intermediate Baseline. Phys.Rev., D78:111103, 2008.

[67] Stephen J. Parke, Hisakazu Minakata, Hiroshi Nunokawa, and Renata Zukanovich Funchal. Mass Hierarchy via Mossbauer and Reactor Neutrinos. Nucl.Phys.Proc.Suppl., 188:115-117, 2009.

[68] Liang Zhan, Yifang Wang, Jun Cao, and Liangjian Wen. Experimental Requirements to Determine the Neutrino Mass Hierarchy Using Reactor Neutrinos. Phys.Rev., D79:073007, 2009.

[69] X. Qian, D.A. Dwyer, R.D. McKeown, P. Vogel, W. Wang, et al. Mass Hierarchy Resolution in Reactor Anti-neutrino Experiments: Parameter Degeneracies and Detector Energy Response. Phys.Rev., D87(3):033005, 2013.

[70] Emilio Ciuffoli, Jarah Evslin, and Xinmin Zhang. The Neutrino Mass Hierarchy at Reactor Experiments now that theta13 is Large. JHEP, 1303:016, 2013.

[71] Shao-Feng Ge, Kaoru Hagiwara, Naotoshi Okamura, and Yoshitaro Takaesu. Determination of mass hierarchy with medium baseline reactor neutrino experiments. JHEP, 1305:131, 2013.

[72] Yu-Feng Li, Jun Cao, Yifang Wang, and Liang Zhan. Unambiguous Determination of the Neutrino Mass Hierarchy Using Reactor Neutrinos. Phys.Rev., D88:013008, 2013.

[73] F. Capozzi, E. Lisi, and A. Marrone. Neutrino mass hierarchy and electron neutrino oscillation parameters with one hundred thousand reactor events. Phys.Rev., D89(1):013001, 2014.

[74] Hiroshi Nunokawa, Stephen J. Parke, and Renata Zukanovich Funchal. Another possible way to determine the neutrino mass hierarchy. Phys.Rev., D72:013009, 2005.

[75] G. Cowan et al. Eur. Phys. J., C71:1554, 2011.

[76] X. Qian, A. Tan, W. Wang, J.J. Ling, R.D. McKeown, et al. Statistical Evaluation of Experimental Determinations of Neutrino Mass Hierarchy. Phys.Rev., D86:113011, 2012.

[77] Mattias Blennow, Pilar Coloma, Patrick Huber, and Thomas Schwetz. Quantifying the sensitivity of oscillation experiments to the neutrino mass ordering. JHEP, 1403:028, 2014.

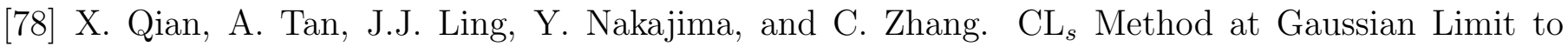
Present Searches. 2014.

[79] Mattias Blennow. On the Bayesian approach to neutrino mass ordering. JHEP, 1401:139, 2014.

[80] Ofer Vitells and Alex Read. A comment on estimating sensitivity to neutrino mass hierarchy in neutrino experiments. 2013.

[81] R. Cousins, J. Mumford, J. Tucker, and V. Valuev. Spin discrimination of new heavy resonances at the LHC. JHEP, 0511:046, 2005.

[82] Georges Aad et al. Observation of a new particle in the search for the Standard Model Higgs boson with the ATLAS detector at the LHC. Phys.Lett., B716:1-29, 2012. 
[83] Serguei Chatrchyan et al. Observation of a new boson at a mass of $125 \mathrm{GeV}$ with the CMS experiment at the LHC. Phys.Lett., B716:30-61, 2012.

[84] Louis Lyons. Discovering the Significance of 5 sigma. 2013.

[85] R. Cousins. The Jefferys-Lindley Paradox and Discovery Criteria in High Energy Physics. 2013.

[86] R. Wendell (Super-Kamiokande), Neutrino 2014, https://indico.fnal.gov/getFile.py/

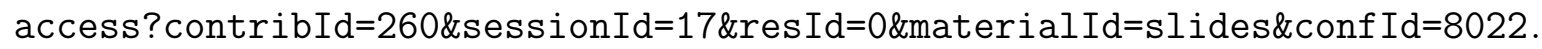

[87] Y. Fukuda et al. The Super-Kamiokande detector. Nucl.Instrum.Meth., A501:418-462, 2003.

[88] Roger Wendell. Atmospheric Results from Super-Kamiokande. 2014.

[89] G. Hill, (IceCube), Neutrino 2014, https://indico.fnal.gov/getFile.py/access?contribId= 237 \&session $\perp d=11$ \&res $\perp d=0$ \&materia $\perp d=s \perp i d e s \& c o n f \perp d=8022$.

[90] C. Walter (T2K), Neutrino 2014, https://indico.fnal.gov/getFile.py/access?contribId= 268\&.session $T d=18 \&$.res $T d=0 \&$ material $T d=s l i d e s \&$.conf $T d=8022$.

[91] A. Sousa, (MINOS/MINOS+), Neutrino 2014, https://indico.fnal.gov/getFile.py/ access? contribld=26/\&session $\perp d=18$ \&res $\perp d=0$ \&materia $\perp \perp d=s \perp i d e s \&$ con $\perp d d=8022$.

[92] Chao Zhang. Recent Results From The Daya Bay Experiment. 2015.

[93] D. Ayres, G. Drake, M. Goodman, V. Guarino, T. Joffe-Minor, et al. Letter of Intent to build an Off-axis Detector to study numu to nue oscillations with the NuMI Neutrino Beam. 2002.

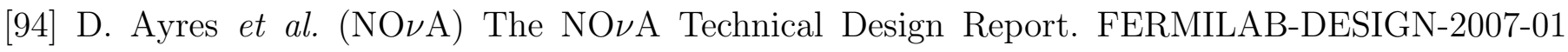
(2007).

[95] A. J. Norman (NO $\nu \mathrm{A})$, https://indico.fnal.gov/getFile.py/access?contribId=264\& session $\perp d=18$ \&es $\perp d=0$ omateria $\perp d=$ s $\perp$ ides $\&$ con $\perp d=8022$.

[96] C. Adams et al. The Long-Baseline Neutrino Experiment: Exploring Fundamental Symmetries of the Universe. 2013.

[97] S.K. Agarwalla et al. The mass-hierarchy and CP-violation discovery reach of the LBNO longbaseline neutrino experiment. JHEP, 1405:094, 2014.

[98] M. Bass et al. Baseline optimization for the measurement of CP violation and mass hierarchy in a long-baseline neutrino oscillation experiment. 2013.

[99] http://lbne.fnal.gov/env-assessment.shtml.

[100] M. Fukugita and T. Yanagida. Baryogenesis Without Grand Unification. Phys.Lett., B174:45, 1986.

[101] W.J. Willis and V. Radeka. Liquid Argon Ionization Chambers as Total Absorption Detectors. Nucl.Instrum.Meth., 120:221-236, 1974.

[102] C. Rubbia. The liquid-argon time projection chamber: A new concept for neutrino detector. CERN-EP/77-08 (1977). 
[103] Eido Shibamura, Akira Hitachi, Tadayoshi Doke, Tan Takahashi, Shinzou Kubota, et al. Drift velocities of electrons, saturation characteristics of ionization and W-values for conversion electrons in liquid argon, liquid argon-gas mixtures and liquid xenon. Nucl.Instrum.Meth., 131:249-258, 1975.

[104] A. M. Kalinin et al. Temperature and electric field strength dependence of electro drift velocity in liquid argon. ATLAS internal note, LARG-NO-058 (1996).

[105] W. Walkowiak. Drift velocity of free electrons in liquid argon. Nucl.Instrum.Meth., A449:288-294, 2000.

[106] S. Amoruso, M. Antonello, P. Aprili, F. Arneodo, A. Badertscher, et al. Analysis of the liquid argon purity in the ICARUS T600 TPC. Nucl.Instrum.Meth., A516:68-79, 2004.

[107] E. Aprile, K.L. Giboni, and C. Rubbia. A Study of Ionization Electrons Drifting Large Distances in Liquid and Solid Argon. Nucl.Instrum.Meth., A241:62, 1985.

[108] S.E. Derenzo, A.R. Kirschbaum, P.H. Eberhard, R.R. Ross, and F.T. Solmitz. Test of a Liquid Argon Chamber with 20-Micrometer RMS Resolution. Nucl.Instrum.Meth., 122:319, 1974.

[109] E. Shibamura, T. Takahashi, S. Kubota, and T. Doke. Ratio of diffusion coefficient to mobility for electrons in liquid argon. Phys. Rev., A20:2547, 1979.

[110] S. Derenzo. Electron diffusion and positive ion charge retention in liquid-filled high-resolution mltistrip ionization-model chamber. LBNL, Group A, Physics note, 786 (1974).

[111] P. Cennini, S. Cittolin, J.P. Revol, C. Rubbia, W.H. Tian, et al. Performance of a 3-ton liquid argon time projection chamber. Nucl.Instrum.Meth., A345:230-243, 1994.

[112] V. M. Atrazhev and I. V. Timoshkin. Transport of electrons in atomic liquids in high electric fields. IEEE Trans. Dielectrics and Electrical Insulation, 5, 450, (1998).

[113] M. G. Boulay and A. Home. Technique for direct detection of weakly interaction massive particles using scintillation timem discrimination in liquid argon. Astro. Part. Phys., 25:189, 2006.

[114] M. Miyajima et al. Average energy expended per ion pair in liquid argon, liquid argon-gass mixtures and liquid xenon. Phys. Rev., A9:1438, 1974. and erratum in A10, 1452 (1974).

[115] Letter of Intent to form an international collaboration for the experimental program at the long-baseline neutrino facility (ELBNF), https://indico.fnal.gov/conferenceDisplay.py? conf $\perp d=9090$.

[116] S. R. Mishra. in Proceedings of the Workshop on Hadron Structure Functions and Parton Distributions, edited by D. Geesaman et al.

[117] A. Bodek, U. Sarica, D. Naples, and L. Ren. Methods to Determine Neutrino Flux at Low Energies:Investigation of the Low $\nu$ Method. Eur.Phys.J., C72:1973, 2012.

[118] M.G. Aartsen et al. Letter of Intent: The Precision IceCube Next Generation Upgrade (PINGU). 2014.

[119] E. Kh. Akhmedov, Soebur Razzaque, and A. Yu. Smirnov. Mass hierarchy, 2-3 mixing and CPphase with Huge Atmospheric Neutrino Detectors. JHEP, 1302:082, 2013.

[120] Ulrich F. Katz. The ORCA Option for KM3NeT. PoS, 2014. 
[122] http://www.ino.tifr.res.in/ino/.

[123] Anushree Ghosh, Tarak Thakore, and Sandhya Choubey. Determining the Neutrino Mass Hierarchy with INO, T2K, NOvA and Reactor Experiments. JHEP, 1304:009, 2013.

[124] K. Abe et al. A Long Baseline Neutrino Oscillation Experiment Using J-PARC Neutrino Beam and Hyper-Kamiokande. 2014.

[125] Yifang Wang. JUNO: current status and future plan. In JUNO Meeting at IHEP, Beijing, China, Jul 2013.

[126] L. J. Wen. Future reactor experiments. In Neutrino 2014 https://indico.fnal.gov/getFile.py/access?contribId=376\&sessionId=15\&resId=0\&materialId= June 2014.

[127] Soo-Bong Kim. New results from RENO and prospects with RENO-50. 2014.

[128] A.B. Balantekin, Henry Band, Russell Betts, Jeff J. Cherwinka, J.A. Detwiler, et al. Neutrino mass hierarchy determination and other physics potential of medium-baseline reactor neutrino oscillation experiments. 2013.

[129] S. Antusch, C. Biggio, E. Fernandez-Martinez, M.B. Gavela, and J. Lopez-Pavon. Unitarity of the Leptonic Mixing Matrix. JHEP, 0610:084, 2006.

[130] Stephen F. King, Alexander Merle, Stefano Morisi, Yusuke Shimizu, and Morimitsu Tanimoto. Neutrino Mass and Mixing: from Theory to Experiment. New J.Phys., 16:045018, 2014.

[131] P. F. Harrison, D. H. Perkins, and Scott W.G. Tri-bimaximal mixing and the neutrino oscillation data. Phys. Lett. B, 530:167, 2002. 